\title{
The influence of anxiety on pain : attentional and physiological mediators
}

Citation for published version (APA):

Janssen, S. A. (1998). The influence of anxiety on pain : attentional and physiological mediators. [Doctoral Thesis, Maastricht University]. Universiteit Maastricht. https://doi.org/10.26481/dis.19981016sj

Document status and date:

Published: 01/01/1998

DOI:

10.26481/dis.19981016sj

Document Version:

Publisher's PDF, also known as Version of record

\section{Please check the document version of this publication:}

- A submitted manuscript is the version of the article upon submission and before peer-review. There can be important differences between the submitted version and the official published version of record.

People interested in the research are advised to contact the author for the final version of the publication, or visit the DOI to the publisher's website.

- The final author version and the galley proof are versions of the publication after peer review.

- The final published version features the final layout of the paper including the volume, issue and page numbers.

Link to publication

\footnotetext{
General rights rights.

- You may freely distribute the URL identifying the publication in the public portal. please follow below link for the End User Agreement:

www.umlib.nl/taverne-license

Take down policy

If you believe that this document breaches copyright please contact us at:

repository@maastrichtuniversity.nl

providing details and we will investigate your claim.
}

Copyright and moral rights for the publications made accessible in the public portal are retained by the authors and/or other copyright owners and it is a condition of accessing publications that users recognise and abide by the legal requirements associated with these

- Users may download and print one copy of any publication from the public portal for the purpose of private study or research.

- You may not further distribute the material or use it for any profit-making activity or commercial gain

If the publication is distributed under the terms of Article $25 \mathrm{fa}$ of the Dutch Copyright Act, indicated by the "Taverne" license above, 
The influence of anxiety on pain: attentional and physiological mediators 


\section{Foto omslag:}

Theo Janssen

\section{Druk:}

Datawyse Maastricht

Het onderzoek in dit proefschrift werd financieel gesteund door de Stichting Gedragswetenschappen (SGW), die wordt gesubsidieerd door de Nederlandse Organisatie voor Wetenschappelijk Onderzoek (NWO; projectnummer 575-86-056).

ISBN $90-9012013-0$ 


\title{
The influence of anxiety on pain: attentional and physiological mediators
}

\author{
PROEFSCHRIFT \\ ter verkrijging van de graad van doctor \\ aan de Universiteit Maastricht. \\ op gezag van de Rector Magnificus " \\ Prof dr. A.C. Nieuwenhuijzen Kruseman, \\ volgens het besluil van het College van Decanen, \\ in het openbaar te verdedigen op \\ vrijdag 16 oktober 1998 om 12.00 uur
}

door

Sabine Anne Janssen 


\section{Promotor:}

Prof. dr. M.A. wan den Hout

Co-promotor:

Dr. A. Arntz

Beoordelingscommissie:

Prof. dr. H.A.J. Struijker Boudier (voorzitter)

Prof. dr. W. Everaerd (Universiteit van Amsterdam)

Prof. dr. H. Flor (Humboldt-Universität zu Berlin, D.)

Dr. M. Peters

Dr. J. Vlaeyen 


\section{Contents}

1.1 The influence of anxiety on pain: elinical and theoretical views 8

1.2 Possible mediators in the influence of anxiety on pain 9 Endogenous opioids 9 Catecholamines 11 Increased muscle tension $\quad 11$ Arousal $\quad 11$ Attention 12

$\begin{array}{lll}1.3 \text { Conclusion } & 14\end{array}$

2.1 Correlational studies $\quad 15$

2.2 Experimentally induced anxiety: early studies 17

2.3 Focus of anxiety 17

2.4 Focus of attention 19

2.5 Stress-induced analgesia in humans 21

2.6 Conclusion and remaining questions 22

3.1 Research questions 25

3.2 Anxiety and pain: attentional and endorphinergic influences 27

3.3 No evidence for opioid-mediated analgesia induced by phobic fear

3.4 No interactive effects of naltrexone and benzodiazepines on pain during phobic fear

3.5 Uncontrollable stress and opioid-mediated analgesia in novice parachute jumpers

3.6 Anxiety and pain: epinephrine-induced hyperalgesia and attentional influences

3.7 Does anxiety lead to less distraction from pain? 
4.1 Answering the research questions 93

Endogenous opioids and attentional focus 93

Endogenous opioids and uncontrollable anxiety 94

Epinephrine-induced hyperalgesia 95

Anticipatory anxiety and distraction from pain 96

Concordance between subjective

and psychophysiological measures 96

4.2 Towards an integrated model 97

Endogenous opioids $\quad 98$

Epinephrine $\quad 99$

Attentional focus 100

4.3. Suggestions for future research $\quad$ 101

4.4. Implications for clinical practice 103

$\begin{array}{ll}\text { References } & 105\end{array}$

$\begin{array}{ll}\text { Summary } & 111\end{array}$

$\begin{array}{ll}\text { Samenvatting } & 113\end{array}$

$\begin{array}{ll}\text { Dankwoord } & 115\end{array}$

$\begin{array}{lr}\text { Curriculum Vitae } & 117\end{array}$ 


\section{Introduction}

Pain is usually regarded as a sensation evoked by noxious external or internal stimuli. Although described as a sensation, it is not in all respects a sensation similar to vision or hearing. Whereas most other sensory modalities are used to explore the environment, providing information on the whereabouts and the nature of environmental stimuli "pain is more immediately linked to certain response patterns, such as withdrawal or recuperative behavicur. This makes it akin to motivational systems such as hunger or thirst (Wall, 1979; Bolles \& Fanselow, 1980). In contrast to other sensations, pain usually has a strong affective component, and is more adequately described in terms of the consequences that a stimulus has on the organism (possibility of tissue damage). These aspects of pain have been integrated in the definition given by the International Association for the Study of Pain (IASP 1979), where pain is described as "an unpleasant sensory and emotional experience, associated with actual or potential tissue damage or described in terms of such damage". Following this definition, a distinction should be made between pain and nociception. Nociception refers to the reception of signals in the central nervous system evoked by activation of specialized sensory receptors (nociceptors) that provide information about tissue damage. Although the perception of pain is obviously related to activation of nociceptors, there is certainly no one-to-one relationship. Not all pain experience is the result of a noxious stimulus or an injury, nor do all noxious stimuli that activate nociceptors necessarily lead to the experience of pain. Classical anecdotes were provided by Beecher (1956), who surveyed wounded soldiers barely noticing their severe wounds while in combat. It seems that pain is a complex and highly subjective phenomenon, that can be influenced by several psychological and physiological factors.

A very influential theory on pain perception has been Melzack \& Wall's gatecontrol theory (1965; 1982). A mechanism in the dorsal horn of the spinal cord was thought to act as a gate, which modulates or blocks nociceptive information to be processed by the central nervous system. This gate, mediating the variable relationship between tissue damage and pain perception, could be operated in several ways. It is further opened by activation of small, fast-conducting fibers which leads to well-localized, distinct pain (AS-fibers), or by activation of slowconducting fibers, from which dull poorly localized pain arises (C-fibers). It is more closed, however, by activation of nonnociceptive Ap-fibers, for instance produced by rubbing the skin, which may in this way inhibit pain arising from $A \delta$ or Cufibers. Apart from these influences from peripheral afferent nerve activity, there is also evidence that central pathways, descending from the brain, modulate the transmission of nociceptive information at spinal cord level. Melzack \& Wall pointed out the possibility that affective and cognitive factors, like anxiety, attention and expectation, exert an influence on pain wia these descending pathways. Their theory has stimulated research and theorizing on the influence of psychological factors on pain perception. 


\subsection{The influence of anxiety on pain: clinical and theoretical views}

It is generally acknowledged that there is a relationship between anxiely and pain, anxiely being an important modulating factor in the perception of pain. Following the IASP definition given above, the emotional response to pain should even be considered part of the pain experience. Anxiety has been described as a complex response characterized by subjective feelings of apprehension and tension, accompanied by or associated with sympathetic physiological arousal (Paul \& Bernstein 1973). Whille there may be a semantic distinction between anxiety, refering to a certain state or trait, and fear, being more stimulus-bound (Marks, 1987), both terms have interchangeably been used in relevant pain research.

In both clinical and theoretical views, increased levels of anxiety are generally believed to be associated with increased pain reports. Accordingly, reduction of anxiety is an important objective in interventions to control pain in clinical settings, for instance by relaxation training, sensational or procedural information, or enhancing perceived control over the stimulus (Chapman \& Bonica, 1983), or by the administration of benzodiazepines (Dellemijn \&. Fields, 1994). Anxiety has been argued to increase pain by stimulating the release of epinephrine at sympathetic nerve endings, which may sensitize or directly activate nociceptors, and by uriggering somatic reflexes through increased muscle tension (Chapman \& Tumer, 1986). In Melzack \& Wall's gate-control theory (1982), anxiety is hypothesized to influence pain perception by further opening the gate at the spinal level via descending pathways. Furthermore, correlational studies have demonstrated a positive relationship between anxiety and pain; the greater the anxiety, the greater is the pain (e.g. Dougher, 1979; Ahles, Cassens \& Stalling, 1987). However, from correlational studies no conclusions can be drawn with regard to the causal direction of this influence. Also, as pointed out by Gross \& Collins (1981), anxiety and pain may be methodologically confounded, since they both lead to general sympathetic physlological arousal and share common response patterns.

Not in all theoretical accounts it is assumed that anxiety necessarily increases pain. Based on his owm experience, and on Beecher's observations of injured soldiers without pain, Wall (1979) proposed an immediate phase after injury in which pain does not always occur. In this phase, anxiety may be present without intensifying pain. Priority is given to behaviour like fighting, escaping or obtaining aid. Wall suggested a link between attention and this prioritizing process, although he denied any additional explanatory power of the concept of attention. in a later phase, pain arises to promote recovery from and treatment of the injury. When anxiety is present in this phase it may contribute to and become almost synonymous with pain. Closely related to the first part of this view, Bolles \& Fanselow (1980) have postulated the idea that fear and pain are two different motivational systems, fear being related to defensive behaviour, and pain leading to recuperative behaviour. In their so-called perceptual-defensive-recuperative model of pain and fear. Bolles \& Fanselow (1980) hypothesize that these motivational systems are mutually inhibiting, fear having priority over pain and inhibiting pain experience to promote fight or flight behaviour. This is illustrated 
by the bulk of animal studies providing evidence for analgesic effects induced by uncontrollable stress (e.g. Maier et al, 1983). The effects are attributed to endogenous opioids, morphine-like peptides in the brain, which are released during threatening situations. Unlike Wall, who states that anxiety and pain are usually two faces of the same coin, with the exception of the phase immediately after injury. Bolles \& Fanselow argue that the typical effect of anxiety on pain is inhibitory. In those circumstances where anxiety is observed to facilitate pain. they attribute this to the effect of prolonged muscle tension or to the mislabeling of anxiety as pain. The latter explanation is related to accounts based on attribution theory, stating that physiological arousal associated with anxlety for pain may increase pain because it is perceived as part of the pain (Nisbett \& Schachter, 1966; Weisenberg et al, 1984). It has also been suggested that not the (mis) labeling of physiological arousal but the focus of attention is critical for the direction of effects of anxiety on pain (Arntz, Dreessen \& de Jong, 1994). In line with the idea that anxiety leads to an attentional allocation towards threatening information (Eysenck, 1988), anxiety for pain would lead to attention towards pain, whereas anxiety for something else would lead to distraction from pain, thereby respectively increasing or decreasing pain.

\subsection{Possible mediators in the influence of anxiety on pain}

Several possible mediators in the influence of anxiety on pain have been mentioned while discussing clinical and theoretical views in this area. Some of the proposed mediators are physiological in nature, such as the release of endogenous opioids or catecholamines during anxiety and the effects of sympathetic activation or muscle tension, others are more psychological in nature, such as the attribution of physiological arousal, or the allocation of attention to pain or to other objects of anxiety. In this paragraph, each of these possible mediators will be described in more detail, discussing the evidence for their potential role in anxiety or pain perception.

\section{Endogenous opioids}

While the analgesic effects of morphine and other exogenous opioids have been known and used by clinicians for a long time, the existence of specific opioid receptors and endogenous, morphine like peptides in the brain has only been demonstrated in the mid-seventies. Since their discovery, the biology and function of endogenous opioids have been extensively studied. Three types of endogenous opioids have been discovered over time, classified on the basis of their different precursers in the brain: the enkephalins (leu-and met-enkephalin). B-endorphin, and dynorphin. Enkephalins and dynorphin are found in the periaqueductal grey, rostroventral medulla, and in the dorsal spinal cord, while B-endorphin is confined primarily to the hypothalamus, projecting to periaqueductal grey and brain stem, and is released from the pituitary into the blood stream as a response to certain stressors. The autonomic nervous system has also been shown to contain enkephalins (Grossman, 1988). While the enkephalins tend to have local effects near the point of release, acting as neurotransmitters, $\beta$-endorphin tends to have systemic effects. All three types of 
endogenous opioids produce analgesia, although the enkephalins and $\beta$ endorphin are more potent than dynorphin. In addition to the three classes of endogenous opioids, there are three major classes of opioid receptors: mu, delta and kappa receptors. The mu-receptor, which seems the major receptor related to analgesic effects, is abundant in central areas for pain control, in periaqueductal grey matter, in brain stem and dorsal spinal cord, and also in the limbic system. The kappa receptor is present in these areas as well, but also in deeper layers of the cerebral cortex, whereas delta receptors are quite generally distributed (Terenius, 1985). In contrast to morphine, which has the highesit affinity for mu-receptors, $\beta$-endorphin and the enkephalins bind to both $\mathrm{mu}$-and delta-receptors, while dynorphin binds primarily to the kappa-receptor (Akil et al., 1984). Although the mu-receptor seems most important in the analgesic effects of opioids, delta- and kappa-receptors may also be involved.

A very important tool in studying the biology and function of endogenous opioids has been the use of opioid antagonists. Opioid antagonists are defined by the characteristic of binding to an opioid receptor site without activating it, thereby blocking the receptor for opioid agonists. Opioid antagonists are typically thought of as inactive in themselves, and functioning only to reverse the effects of opioids. Therefore, the reversal of analgesia by an opioid antagonist is generaly accepted as evidence that the analgesia was caused by endogenous opioids. The most widely used opioid antagonist is naloxone. It has highest affinity for mureceptors, being about 10 times more potent to bind to mureceptors than to kappa- or delta-receptors. Although it is traditionally viewed as a pure antagonist, there are indications that naloxone by itself has analgesic properties, especially in low doses (Buchsbaum, Davis \& Bunney, 1977; Lewine, Gordon \& Fields, 1979; Stacher et al., 1988).

Because for years exogenous opioids like morphine had been useful in pain

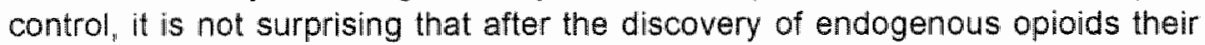
analgesic effect received most attention. However, next to their role in pain perception, endogenous opioids have been attributed a rolle in learning and memory for aversive events (Rose \& Orlowski, 1983), attention (Arnsten et al., 1983), euphoria (Hennig, Laschefski \& Opper, 1994), anxiety and approach behaviour (Merluzzl et al. 1991; Arntz, Merckelbach \& de Jong, 1993) and inhibition of extreme sympathetic activation (Bouloux, Grossman \& Al Damluji. 1985). The endagenous opioid system seems to be part of a more general stress response, related to physiological and behavioral adaptation during stressful situations (Cohen. Pickar \& Dubois, 1983; McCubbin, 1993). It should be mentioned, however, that the term stress has been used to cover a wide range of situations. In animal research, stressful situations as diverse as cold-water immersion, centrifugal rotation, food deprivation, electricall shock, heat exposure, exposure to a natural predator and social isolation were shown to induce analgesic effects that were more or less reversed by naloxone (Rogers \& Randall, 1988). A precondition for stress-induced analgesia appears to be that the stress is uncontrollable or inescapable (Maier et al, 1983). Although the idea that uncontrollable stress or fear induces analgesia is hardly challenged in the field of animal research on endogenous opioid-mediated analgesia, it is only confirmed by few studies in humans. In humans, a naloxone-reversable analgesia 
followed nociceptive stimuli (Willer Ernst, 1986), long-distance running (Janal et al., 1984), clinical pain (Cohen, Pickar \& Dubois, 1983), and a combat video in Vietnam veterans with PTSD (Pitman et al. 1990).

\section{Catecholamines}

It has been hypothesized that anxiety increases pain through the release of catecholamines, peripherally sensitizing or even stimulating nociceptors (Chapman \& Turner, 1986). This idea is supported by research on Sympathetically Maintained Pain (SMP), a chronic pain state that may arise after damage to one of the major peripheral nerves. It is characterized by severe burning pain which may persist for years, but cain be alleviated by sympathetic block or sympathectomy (Wesenfeld-Hallin \& Hallin, 1984). Therefore, the sympathetic nervous system is believed to importantly affect pain perception. However, animal studies have shown that sympathetic stimulation alone does not excite nociceptors (Shea \& Perl, 1985; Barasi \& Lynn, 1986). It seems that, only after receptive field sensitization or peripheral nerve injury, nociceptor discharge is markedly facilitated by sympathetic stimulation or local norepinephrine injection (Roberts \& Elardo, 1985; Hu \& Zhu, 1989; Sato \& Perl, 1991). In humans, sensitization has been experimentally induced by toplcal application of capsaicin (an algogenic compound, derived from red peppers) which produces a temporary inflammation and a hyperalgesia to heat or mechanicall stimuli. On capsaicintreated skin, peripheral injection of norepinephrine resulted in lower pain thresholds than injection of placebo (Drummond, 1995). However, pain thresholds in normal skin were also lowered by (nor)epinephrine, although effects were smaller than in capsaicin-treated skin (Drummond, 1996; Choi \& Rowbotham, 1997). It therefore seems that particularly when skin is injured or sensitized, sympathetic activation or the associated rise in peripheral catecholamines increases pain. Thus, pain may be increased during anxiety due to the release of catecholamines.

\section{Increased muscle tension}

Anxiety for pain often leads to increased muscle tension in the areas associated with the pain. Muscle tension caused by anxiety may contribute to pain by a positive feedback loop, in which nociception fosters increased muscle tone or ischemia near the wound, eventually activating muscle nociceptors (Chapman \& Turner, 1986). Bolles \& Fanselow (1980) mention increased muscle tension as an explanation for those instances in which anxiety increases pain, contrary to their theory that anxiety generally inhibits pain. However, they admit that not all pain increases observed during anxiety can be attributed to increased muscle tension. Where for instance is the tooth muscle that increases pain at the dentist in the patient with dental anxiety? Although it may sometimes contribute to the pain increased muscle tension does not seem to be a satisfactory explanation for all pain-increasing effects of anxiety.

\section{Arousal}

Apart from being associated with the release of catecholamines which may sensitize nociceptors, physiological arousal during anxiety has been arguled to 
influence pain in less peripheral ways. According to Groves \& Thompson's dualprocess theory of habituation (1970), based on experiments with spinal reflexes, in the neural pathway between stimulus detector and response effector a gradual decline in efficiency builds up, leading to a decrement in responding. Arousal is believed to influence this process, high arousal being associated with sensitization to stimulation, resulting in stronger responses and less habituation However, the way arousal is defined by Groves \& Thompson may lead to circular reasoning, since arousal is for instance equated with the enhanced tendency to respond. Arousal is probably best defined by autonomic activation, which manifests itself in autonomic measures like skin conductance level and heart rate. Although Eysenck (1980) makes a clear distinction between autonomic activation and cortical arousal, he states that "autonomic activation, as in fear, produces higher degrees of cortical arousal, and hence lowers sensory thresholds for pain, and also lowers pain tolerance".

Another way in which arousal or physiological activation may influence pain is through the mechanism of attribution. According to attribution theory (Schachter \& Singer, 1962), an emotional state is the result of the interaction between two components: 1) physiological arousal, and 2) a cognition about the arousing situation. Arousal would only determine the intensity of emotion, while the cognition, causally attributing the arousal to an emotional source, determines which emotion, if any, will be experienced (Reisenzein, 1983). This cognitive process is assumed to occur quite rapidly and to be largely unconscious in nature. While usually the cause of arousal and the cognitive attribution coincide, sometimes arousal is misattributed. An experiment by Nisbett \& Schachter (1966) showed that pain-related anxiety increases the likelyhood that arousal will be attributed to the pain, thereby increasing pain experience and reducing tolerance. Thus, physiological arousal associated with anxiety for pain may increase pain because it is labeled as pain. Weisenberg et al. (1984) proposed that, through the attribution of arousal to pain, anxiety which is relevant to the pain-inducing stimulus would exacerbate pain, whereas anxiety which is irrelevant to the pain would not, or would potentially even reduce the experience of pain, since arousal would be attributed to other factors.

\section{Attention}

"Altention implies witdrawal from some things in order to deal effectively with others" (William James, 1890). This remark by one of the founders of modern psychology stresses the selective aspect of attention. Attention has been conceptualized as a selective filter at an early level of processing that heips to cope with the limited capacity to process information (Broadbent, 1958; Kahneman, 1973). Later, a distinction was made between automatic and controlled processing, of which only the latter would draw upon limited processing resources (Shiffrin \& Schneider, 1977). A recent, more functionalistic account emphasizes the involvement of attention in the coordination between sensory input and behaviour (Allport, 1993). Selection in this view does not necessarily mean that unselected information is not processed, but that it is inhibited in favour of ather information. While in principle the capacity of the processing system may be unlimited, selection of information serves to facilitate some 
particular outcome, assigning priority for motor acts, consciousness and memory Attention seems to be an important factor in pain perception and is integrated in several theories on pain (Melzack \& Wall, 1982; Leventhal \& Everhait "1979). A sudden pain stimulus is usually associated with the direction of attention towards the source or location of the pain, and with the partial or total breakdown of ongoing activities. This interruptive property, which is central to pain, may serve to promote either defensive or recuperative behaviour. Somelimes, however, pain has to compete for attention with other stimuli of high priority, such as another object of threat. A lot of research has been dedicated to studying effects of anxiety on attentional allocation (Eysenck, 1988; MacLeod \& Mathews, 1988). In general, anxiety seems to be associated with an attentional allocation towards the object of threat. Thus, anxiety related to pain would be expected to lead to an increase in attention towards the pain, whereas anxiety related to another object of fear would be expected to distract attention from pain. The focus of attention has been hypothesized to be an important mediator in the influence of anxiety on pain: whereas anxiety related to pain would increase pain by the allocation of attention towards pain "pain-irrelevant anxiety would inhibit pain by distracting attention from pain (Arntz, Dreessen \& Merckelbach, 1991; Arntz \& de Jong, 1993; Amtz, Dreessen \& de Jong, 1994).

The idea that the influence of anxiety on pain is mediated by attentional focus leans heavily on the assumption that distraction from pain leads to a reduction in pain with respect to attention focused on pain. There is strong evidence that attention to bodily symptoms increases the perceived intensity of these symptoms (Pennebaker \& Skelton, 1978; Pennebaker \& Lightner, 1980). Intuitively "it seems obvious that distraction from pain should reduce pain, and people often use this strategy spontaneously. In clinical interventions, instructions to distract attention from pain are successfully used as a means for coping with pain-produced distress (Tan, 1982). In line with this, several experimentall studies have shown that a diversity of distraction tasks led to a reduction in pain intensity or an increase in pain tolerance (McCaul and Malott, 1984). The explanation given for pain-inhibiting effects of distraction is that when an attentionally demanding task is performed, it draws upon attentional resources, leaving less capacity for the processing of the pain. However "while the focus of attention may have an impact on pain perception, characteristics of the pain may also influence attentional allocation. It has been hypothesized that distraction would only be effective with low intensity pain. whereas at greater levels of intensity the pain would be given priority, and attention would automatically be turned towards the pain (McCaul \& Haugtvedt, 1982; Hodes et al. 1990). There is evidence that, with high intensity pain, attention to the sensory aspects of pain might be more beneficial, redefining them or processing them in an objectivelanalytic way (Ahles, Blanchard \& Leventhal, 1983). However, contradictory results have been found, indicating either that redefinition is not superior to distraction, even with high intensity pain (Brewer \& Karoly, 1989), or that distraction does not affect pain at all (McCaul, Monson \& Maki, 1992). Recent accounts warn against the strong commonsense belief that distraction always inhibits pain, and propose that the circumstances that contribute to pain-inhibiting effects of distraction be more thoroughly experimentally studied (Leventhal, 1992; Eccleston, 1995) 


\subsection{Conclusion}

Considering the various theories discussed above, there appears to be a lot of controversy about the influence of anxiety on pain. While some theories suggest that anxiety has pain-increasing effects, others propose that anxiety inhibits pain. Both directions of influence can be defended on the basis of certain mechanisms that are believed to play a role during anxiety, some of which are physiological (endogenous opioids, catecholamines) and some of which are psychological in nature (attribution, attention). To address the question whether these mechanisms actually exert effects on pain experience during anxiety, empirical evidence should be discussed from studies investigating the direction and mechanisms of the influence of anxiety on pain. 
From the previous chapter it is obvious that theorists are not unanimous regarding the direction of an influence of anxiety on pain. The various mechanisms that have been proposed as mediators also yield different predictions for the direction of an effect. What is the empirical evidence avallable from studies investigating the influence of anxiety on pain in humans?

\subsection{Correlational studies}

Correlational studies generally indicate a positive relationship between anxiety and pain. Clinically diagnosed anxiety was found to be associated with relatively low pain thresholds (Hall \& Stride, 1954). Valicer (1978) found that spontaneous pain, rated by medical and surgical patients in a hospital, correlated positively with measures of hospital stress. Ahles, Cassens \& Stalling ( 1987 ) found that high state anxiely was associated with higher ratings of spontaneous pain in students. Also, Dougher (1979) found that trait anxiety in students was associated with lower pain thresholds to a pressure pain stimulator, but not with differences in discriminability (used as a measure of the sensory component of the pain as opposed to response bias). Malow, West \& Sutker (1987) found a decrease in pressure pain threshold, and a decrease in discriminability in highly anxious compared to less anxious substance abusers. Also, Malow, West \& Sutker (1989), found that among highly anxious detoxified substance abusers receiving treatment, those showing a decrease in anxiety after treatment rated pressure pain lower than those showing no decrease in anxiety. Furthermore, Klepac t al. (1980) found that high dental fear subjects retrospectively rated tooth shock (but not arm shock) more painful than low dental fear subjects, Wardle (1983) found that sensation information during dental treatment reduced both anxiety and pain ratings as compared to nomal treatment, and Passchier at al. (1992) found positive correlations between anticipatory anxiety for venipuncture and reported needle pain. Although Arntz, van Eck \& Heijmans (1990) found no relation between dental anxiety and dental pain, and Kent (1984) even found a negative association, the bulk of these correlational studies suggests that anxiety increases pain. However, it has already been stated that the disadvantage of correlational studies is that they are not conclusive about the causal direction of the relation. Although it is assumed that anxiely exacerbates pain. in many cases the positive correlation may just as well be attributed to an increase in anxiety as a result of the aversiveness of the pain experience. Also, a third wariable may be responsible for any positive correlation. Therefore, to determine causal relations, studies are required in which anxiety is experimentally manipulated. 


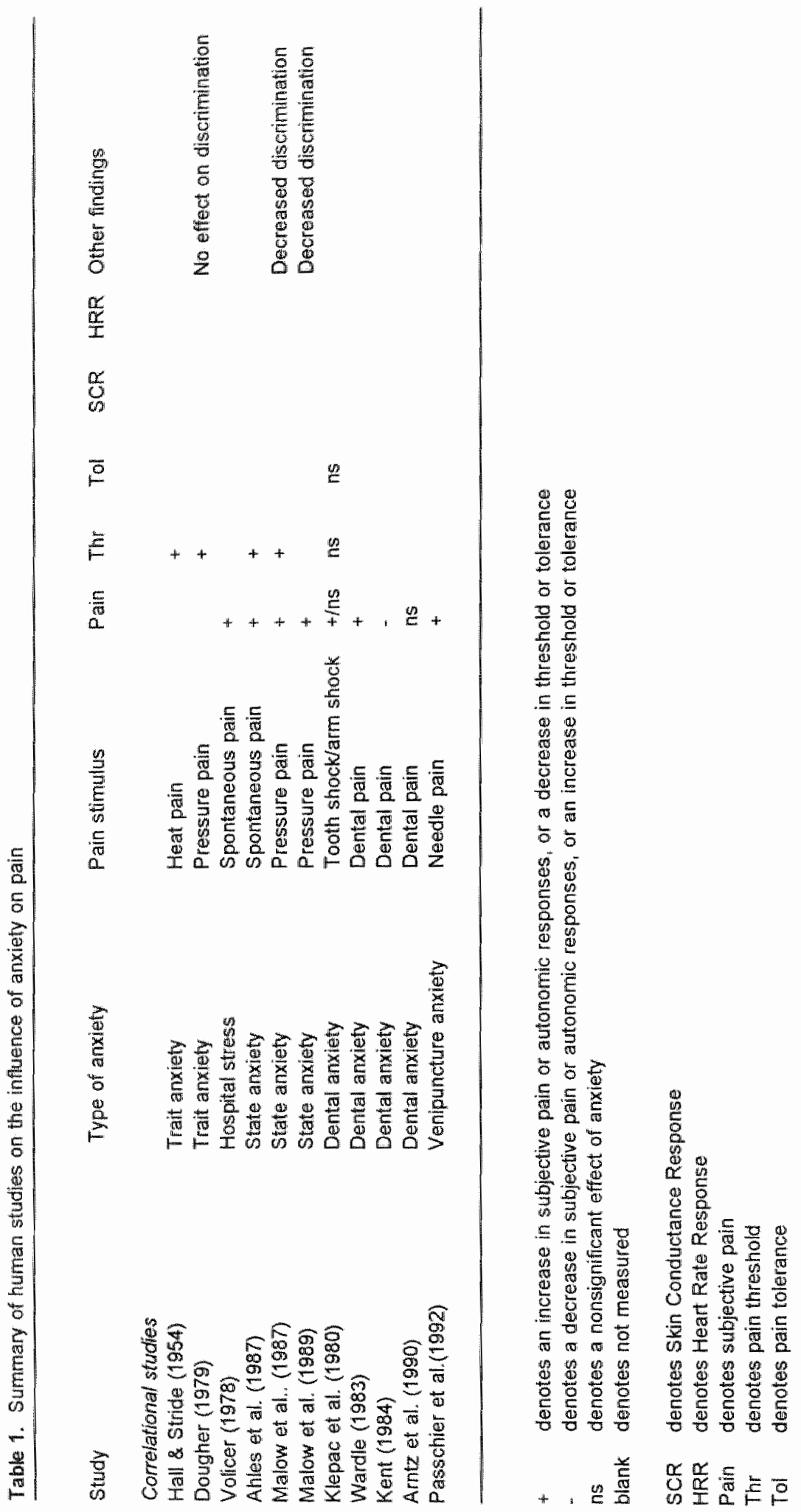









\subsection{Experimentally induced anxiety: early studies}

Beecher (1959) claimed that, since experimental pain is usually unaccompanied by anxiety, findings from experimental studies could not be directly applied to clinical pain, of which anxiety is an important aspect. To make pain research more generalizable to real life situations, some researchers tried to induce anxiety and investigate its effect in the laboratory. Usually, the objective was to manipulate anxiely related to the pain. Haslam (1966), who threatened subjects with an electric shock, found that anxiety decreased heat pain threshold for radiant heat. In line with this, Bobey \& Davidson (1970) demonstrated that a procedure designed to reduce state anxiety by relaxation increased pain thresholds for heat pain and pressure pain. Contrary to their prediction, however, they found higher thresholds in a group who had listened to an "anxiety" tape of a woman in child labour than in a control group. Malow (1981), who like Haslam (1966) threatened subjects with an electric shock, found no influence on their thireshold for pressure pain stimulation, and found a decrease in discriminability in the threat condition as compared to the no threat condition, as well as a decrease in their tendency to report perceived sensations as painful. Schumacher \& Velden (1984) did the same, except for measuring the effect on electrical stimulation, and found no effect on pain threshold, but a tendency to rate stimuli as more painful in the threat than in the no threat condition. Also, they found decreased discriminability as a result of anxiety in the lower, and increased discriminability in the upper range of electrical stimulation intensity, leading them to suggest that the discrepancy between studies could be explained by the use of a not large enough range of intensities in other studies. Malow, who used both subjective and physiological measures of anxiety as a manipulation check, attributed contradictory results to the absence in several studies of an independent verification of whether anxiety was actually induced. Thus, although these experimental studies partly confirmed indications from correlational studies that anxiety increases pain, the results do not seem to clearly point in one direction.

\subsection{Focus of anxiety}

Most studies reported above, both correlational and experimental, were concerned with pain-related anxiety. However, while usually anxiety induction and pain measurement were related to the same pain stimulus, in Malow"s (4981) study, subjects were exposed to a different pain stimulus than the one they feared. This led Dougher, Goldstein \& Leight (1987) to suggest another explanation for the discrepancy between findings, stressing the role of the focus of anxiety. In their study, pain specific anxiety or general anxiety was induced by means of a modified Velten mood induction (Velten, 1968), subjects reading aloud either pain-specific events or generally fearful events. Only pain specific anxiety induction lowered pressure pain threshold and tolerance compared to a neutral condition. It was hypothesized that anxiely related to the pain stimulus leads to an increase in the experience of pain, whereas anxiety related to something else does not. 
A similar suggestion was made by Weisenberg et al. (1984), who studied the importance of the focus of anxiety by inducing pain-related anxiety, anxiety related to a learning task, or anxiety for both. Two rows of green lights were shown, with at the far end of each a red light. Anxiety for the electrical pain stimulus was induced by telling subjects that the upper red light meant that the pain was unsafe, whereas anxiety about the learning task was induced by telling subjects that the lower red light meant that the subject was not performing at the expected level. The lights would light up one after the other, with the red light being almost but never quite reached. In the low anxiety condition subjects were asked to focus attention on the pain, on the task, or on both, and were toid that the lights were only there for the experimenter to receive information about the apparatus. Subjects who were made anxious about the learning task obtained the lowest skin conductance responses to the pain. Furthermore, among subjects who were anxious, there was a trend for subjective pain to be lowest in the task anxiety condition and highest in the anxiety for both condition, with the pain anxiety condition in between. Although these data suggest differential effects on pain for pain-relevant and pain-irrelevant anxiety, it cannot be excluded that both relevant and irrellevant anxiety increased pain, since 1) not only pain, but also anxiety was lower in the task focus condition and 2) pain ratings were not reported for subjects in the low anxiety conditions, making a comparison with low anxiety impossible. Al-Absi \& Rokke (1991) did a similar study, in which relevant anxiety was manipulated by high or low anxiety provoking information about a cold pressor (hand in icewater), and irrelevant anxiety by high or low anxiety provoking information about a potential shock that was never given. They found a significant interaction effect between level and source of anxiety. with high anxiety about the cold pressor leading to sligtly higher cold pressor pain ratings than low anxiety, and high anxiety about the shock leading to slightly lower cold pressor pain ratings than low anxiety. Although high anxiety about the shock did not significantly decrease pain compared to a control condition, the conclusion was that at least anxiety does not necessarily increase pain, but that differential effects may occur depending on the focus of anxiety.

Both Weisenberg et al. (1984) and Al-Absi \& Rokke (1991) proposed that attributional processes might play a medlating role in the influence of anxiety on pain. Some evidence for this idea was provided by Nisbett \& Schachter (1966), who showed that subjects in a low state of fear who could attribute signs of arousal to a placebo reported electrical shocks to be less painful and tolerated higher levels of shock than subjects who were unable to attribute their arousal to an external source. Subjects who were made anxious by telling them that the shock would be very painful, however, did not show this difference, suggesting that pain-related anxiety increases the likelyhood that arousal will be attributed to the pain. If the physiological arousal associated with anxiety is labelled as pain, then it may exacerbate the pain experience, while when subjects attribute their arousal to a threatening stimulus that is not related to the source of the pain, they will be more tolerant of the pain

However, Cornwall \& Donderi (1988) found evidence that somelimes not only pain-relevant, but also pain-irrelevant anxiety is assaciated with an increase in pain responsivity. They gave subjects one of three different instructions before 
pressure pain stimulation: 1) slandard instructions 2) standard instructions $+a$ pain warning and 3) standard instructions + a warning about a stressful interview. Although there were no differences in threshold or tolerance for pressure pain, pain ratings and heart rate during pain in the anxiety conditions were found to show an increase as compared to the no anxiety condition, irrespective of whether pain was rated by the pain warning group (pain-relevant anxiety) or by the group anticipating a stressful interview (pain-irrelevant anxiety). These results indicate that the relationship between anxiety and pain is not simply dependent on the focus of anxiety. Cornwall \& Donderi suggested that, instead of an attributional model, a perceptual disruption model would better fit the data. During anxiely, the discriminability among pain stimuli may be reduced, and attention may automatically be focused on the most salient aspects of the experience. Usually, this will be the pain, leading to a pain increase because attention is focused on the pain stimulus. Since in previous research the focus of attention may have varied, differences in attentional focus may have been responsible for contradictory effects of anxiety on pain.

\subsection{Focus of attention}

Arntz, Dreessen \& Merckelbach (1991) hypothesized that attentional instead of attributional processes may have accounted for the observed differential effects of pain-relevant and pain-irrelevant anxiety. Not the focus of anxiety, but the focus of attention may be the most important mediating factor in the influence of anxiety on pain. Whereas in most previous studies possible attentional effects were not controlled for, Arntz, Dreessen \& Merckelbach (1991) independently manipulated anxiety and the focus of attention. In a group of spider phobic subjects, pain-related anxiety was induced between-subjects by pretending that something was wrong with the electrical shock apparatus or warning the subject that the stimulus might suddenly become stronger. Pain-irrelevant anxiety was induced by confronting the subject with a spider. Since in the first anxiety condition the focus of attention was likely to be primarily on the pain, and in the second condition primarily on the spider, two different low anxiety conditions were included, equated to the high anxiety conditions with regard to the focus of attention. In one condition, subjects were asked to concentrate on the pain and describe it afterwards. In the other condition, subjects were distracted by a neutral video (L.A.Law) and were asked to try not to let themselves be distracted by the pain stimuli. No effect of high versus low anxiety was found on subjective pain and skin conductance responses due to electrical stimulation, whereas the focus of attention proved to have a significant effect on both, irrespective of anxiety, with stronger pain responses when attention was focused on pain than when it was distracted from pain. This was viewed as evidence that the focus of attention mediates effects of anxiety on pain. However, this study has been a spidici in stimulus in the high anxiety/attention condition, and anxiety related to the pain spider phobics was dien/attention condition. Therefore, another study with design, the spider was (Arntz \& de Jong, 1993), in which, in a within-subject 
the high anxiety/distraction condition subjects were confronted with a spider and asked to do several exposure exercises, in the high anxiety/ attention condition subjects were asked to concentrate on the pain stimulus while the spider was placed as near to them as they could tolerate. In the low anxiety conditions, subjects were asked to watch a neutral video (distraction) or to concentrate on the pain stimulus (attention). Again, attention led to stronger pain ratings than distraction, whereas anxiety had no effect. On skin conductance responses, however, attention manipulation had the opposite effect, with attention leading to lower responses than distraction, but only during the first two of the four stimuli given. This was attributed to the more surprising aspects of the stimulus when the subject is distracted than when the subject is waiting for it, skin conductance being sensitive to the suddenness as well as to the intensily of the pain. This proposed effect of suddenness may not have been found in Arnt $z_{i}$ Dreessen $\&$ Merckelbach (1991) since skin conductance response in this study was averaged over 20 stimuli.

Subsequently, Arntz, Dreessen \& de Jong (1994) performed an experiment to directly test the relative explanatory power of the focus of anxiety and the focus of attention. In a pain-irrelevant anxiety/ attention condition anxiety was again induced by the presence of a spider. Subjects in this condition, as well as in a low anxiety/ attention condition, were asked to concentrate on the pain. Further " there were two distraction conditions: one in which subjects were engaged in several exposure exercises (pain-irrelevant anxiety/distraction), and one in which they were asked to watch a neutral video (low anxiety/distraction). Pain-relevant anxiety was induced by telling subjects that the level of electrical stimulation could suddenly increase. It was considered impossible to create a pain-relevant anxiety condition with attention distracted from pain, since this would require contradictory instructions. Attention turned out to increase pain ratings as compared to distraction, irrespective of whether pain-relevant, pain-irrelevant or no anxiety was induced. Again, skin conductance responses diverted from subjective pain, showing no effect of attention manipulation, but a heightened response during pain-relevant anxiety as compared to the other conditions. Although this effect on skin conductance seems to indicate that the focus of anxiety has some influence on autonomic pain responses, it may also be viewed as a response to the uncertainty of the stimulus (about occurrence or intensity) rather than to the intensity of the pain. Taken together, with regard to subjective pain, the three experiments described above strongly suggest a primary influence of attentional focus.

The idea of attentional focus as a mediator in the influence of anxiety on pain provides an explanation for many contradictory findlings. With pain-related anxiety, it is plausible that attention is directed primarily towards the feared pain stimulus. In Klepac et al. (1980) dental anxiety may have increased attention towards tooth shock, but not arm shock. In Bobey \& Davidson (1970) the tape with screams of a woman in labour may have increased pain thresholds by distracting attention from pain, and in Malow (1981) and Weisenberg et al. (1984) the absence of a pain increase during anxiety may have been due to distraction from pain by the lights warning the subject for a noxious shock. Similarly, the pain inhibiting effects found with the learning task in Weisenberg et al. (1984) and the threat of a shock 
in Al-Absi \& Rokke (1991) may be explained by assuming that attention was distracted from the pain stimulation. More evidence for distractive effects of anxiety not directly relevant to the pain comes from Carter. McNeill \& Reed (1991), who found that subjects experiencing pressure pain while watching a video of someone being tortured with dental implements had lower heart rates and escaped from the situation at a later point in time than subjects only experiencing pressure pain. A study by McNell \& Brunetti (1992) suggested that even pain-relevant anxiety does not always increase pain responsivity, since subjects' heart rate during listening to pain scripts (involving descriptions of pain sensations) was unaffected by adding fearful descriptions about corresponding polemtially painful situations. However, in this study there was no actuall pain stimulus to attend to, and heart rate may have reacted equally to the pain information as to the siluational information.

Interestingly, in those studies showing a pain reduction associated with painirrelevant anxiety, the object of threat was actually present during the pain situation (Weisenberg el al., 1984; Al-Absi \& Rokke, 1991; Carter, McNeill \& Reed, 1991). When anxiety is related to a threat other than pain and the threat is present during the pain situation, this aspect of the situation becomes more salient than the pain and distracts attention from pain. However, in Cornwall \& Donderi (1988), the pain-irrelevant anxiety-induction involved the anticipation of a stressful interview immediately after the painful stimulation, but not during pain. In the absence of another object of threat, attention might automatically be focused on the pain, being the most salient and threatening stimulus present. This may also account for the results of a more recent study by Caceres \& Burns (1997), in which a mental stress task, consisting of forced mental arithmetic with negative feedback, lowered pain threshold and tolerance to a subsequent cold pressor in subjects showing high physiological reactivity as compared to subjects showing low physiological reactivity during the mental stress task. The mental task did not concide with the pain, but preceded the pain measurement, and anxiety or stress may have led to the direction of attention towards the pain. Likewise, the positive relationship between general anxiety and pain found in some correlational studies may be understood in the light of an inclination during an anxious state to attend to the pain in the absence of other threatening stimuli.

\subsection{Stress-induced analgesia in humans}

There is ample evidence from animal studies that endogenous opioids released during anxiety can have pain-inhibiting effects (Watkins \& Mayer, 1982). In human research dealing with the influence of anxiety on pain, however, the possibility of an endogenous opioid-mediated analgesia is often ignored, perhaps due to the widely held belief that anxiety increases pain. Furthermore in those cases that a pain inhibition was reported it appeared to be mediated by attentional factors rather than by endogenous apioids.

A few studies with humans investigated endorphinergic influences on pain directly by administering the opioid antagonist naloxone. Willer \& Ernst (1986) repeatedly stressed subjects by giving them either noxious or tactile footshocks, while during rest periods in between these stress periods knee muscle reflex and 
subjective pain were measured to milder electrical stimulation at the sural nerve. Subjective pain showed a decrease and nociceptive reflex threshold an increase as a function of repeated stress, which could be reversed by administering naloxone, an endogenous opioid blocker. Therefore, the observed analgesia was attributed to the release of endogenous opioids. When subjects were given the benzodiazepine diazepam to reduce anxiety, the analgesic effects were much smaller, which, although it does not entirely prove a causal role of anxiety, at least suggests that anxiety and not pain was responsible for the endogenous opioid effects. This would be consistent with evidence from animal studies that, while analgesic effects are usually induced by physiological stressors, it does not seem to be the painful stimulation per se, but the psychological stress over its uncontrollability that induces opioid activity (Maier et al, 1983). Although in Willer \& Ernst (1986) pain stimulation was used as a stressor, more cognitive stressors have also been shown to induce a naloxone-reversable analgesia. Bandura et al. (1988) found that subjects with induced low perceived self-efficacy on a stressful mathematical task showed an increase in pain tolerance for a cold pressor, which was reversed by naloxone, whereas subjects with high perceived self-efficacy did not show these effects. In Pitman et al. (1990), Vietnam veterans with postraumatic stress disorder and control subjects were shown a combat video fragment from the film Platoon. After watching the video, they demonstrated a naloxone-reversable decrease in subjective pain for a heat pain stimulus compared to a neutral video, whereas control subjects even showed an increase (curiously enough also reversed by naloxone). Thus, there is some evidence that in humans, like in animals, endogenous opioids inhibit pain during anxiety or stress-inducing situations.

\subsection{Conclusion and remaining questions}

The studies reviewed in this chapter suggest that pain-related anxiety usually leads to an increase in pain, whereas pain-irrelevant anxiety, provided that the object of threat is immediately present, usually leads to a decrease in pain compared to no anxiety. The crucial factor mediating effects of anxiety on pain, however, does not seem to be the focus of anxiety, but the focus of attention, which has been argued to explain many contradictory results. Next to strong indications for a rolle of attentional focus, however, some studies in humans also demonstrated endogenous opioid-mediated analgesic effects on pain during anxiety or stress. This raises the question how these effects of endogenous opioids and attentional focus relate to each other as mediators in the influence of anxiety on pain. A pain increase during anxiety does not necessarily mean that endogenous opioids did not play a role. since physiological factors may sometimes be overruled by the strong effect of attentional focus. Although endogenous opioids would be expected to inhibit pain, anxiety related to the pain may result in such strong attentional allocation to the pain stimulus that the experience of it is increased. Vice versa, physiological inhibitory mechanisms may add little to the inhibitory effect of distraction. It is also possible that attentional focus and endogenous opioids exert their effects in different types of threatening situations. 
Another unclarity concerns the role of catecholamines released during anxiety. Although in human studies on anxiety and pain catecholamine release or sympathetic activation has sometimes been referred to as possible mediators of pain-increasing effects of anxiety, this idea has never been directly tested. While there is evidence from studies in the field of Sympathetically Maintained Pain that catecholamines may exacerbate pain, these studies usually involve unnaturally high quantities of peripheral catecholamines.

Finally, although distraction from pain seems to explain most effects of painirrelevant anxiety, it is not always clear how altentional focus explains the pain increase that is often found during pain-relevant anxiety with respect to no anxiely. While attention is probably more focused on pain with pain-relevant than with pain-irrelevant anxiety, attention to pain may not be expected to differ much between pain-relevant and no anxiety when there are no other things to attend to.

It is apparent that different lines of research exist with respect to the influence of anxiety on pain, which are only minimally related. In human research, often indicating pain-increasing effects of anxiety, only seldomly any reference is made to the literature on stress-induced analgesia. Vice versa, studies in which naloxone is administered to demonstrate a possible endogenous opioid-mediated analgesia, usually cite only animal research on stress-induced analgesia and ignore the evidence from human studies that anxiety may exacerbate pain. In studies on Sympathetically Maintained Pain, no reference at all is made to the possible repercussions on the relation between anxiety and pain. Further research is needed in which the relative importance of physiological factors, particularly endogenous opioids and catecholamines, and attentional mechanisms is investigated. Given the observed strong effects of attention, it seems advisable to control for attentional focus when investigating influences of anxiety on pain. The present dissertation was intended to provide more insight into the relative role of endogenous opioids, catecholamines and attentional focus as mediators in the influence of anxiety on pain. 


\section{Experimental studies}

\subsection{Research questions}

The following research questions will be addressed in the present dissertation:

1) What is the role of endogenous opioids and attentional focus in the influence of anxiety on pain?

In section 3.2 and 3.3 it was tested whether during high phobic anxiety endogenous opioids have a pain-inhibiting effect with respect to low anxiety, even when controlled for focus of attention. This pain inhibition was expected only in the placebo condition, but not, or to a lesser degree, in conditions in which an opioid antagonist (naltrexone/ naloxone) was given. Furthermore, in accordance with previous studies, attentional focus was expected to have an effect on pain, irrespective of anxiety condition, with attention focused on pain resulting in more pain than distraction from pain.

2) Do endogenous opioids inhibit pain in uncontrollable threat situations in humans?

In section 3.4 the hypothesis was tested that endogenous opioids inhibit pain during a relatively uncontrollable 'flooding-like' confrontation with a phobic object. If this is true, naltrexone would lead to a pain increase with respect to placebo. Also, the reduction of anxiety by means of the administration of a benzodiazepine should result in a smaller endogenous opioid release and therefore in an increase in pain compared to placebo. If, however, no endogenous opioids are released and anxiety increases pain, naltrexone would be expected to have no effect, whereas a benzodiazepine would be expected to lead to a pain inhibition compared to placebo.

In section 3.5 an uncontrollable stressor outside the lab was chosen to study an endogenous opioid-mediated analgesia. It was tested whether, immediately after a first-time parachute jump, subjects receiving naloxone would show a pain increase with respect to subjects receiving placebo.

3) Does epinephrine in natural doses exert hyperalgesic effects?

In section 3.6 epinephrine was systemically infused in doses comparable to those during an anxious state. It was hypothesized that epinephrine would show a dose-dependent hyperalgesic effect with respect to placebo on several pain measures. Since there is evidence that effects may be stronger on sensitized skin, one measure was taken on both sensitized and on normal skin. To investigate the relative role of attention and epinephrine, attentional focus was aiso manipulated. 
4) Does anxiefy lead to less distraction from pain due to a disturbance in attention regulation?

In section 3.7 it was hypothesized that pain-irrelevant, anticipatory anxiety increases pain with respect to no anxiety due to the allocation of attention to pain, which may only be manifest when there is competition for attention. Subjects experiencing pain during anticipatory anxiety were expected to profit less from being in a distracting environment than non-anxious subjects. 


\title{
Anxiety and pain: attentional and endorphinergic influences
}

\begin{abstract}
Summary
The rolle of attentional mechanisms and endogenous opioids in the influence of anxiety on acute pain sensation was investigated. Forty-five spider phobics received mild ly painful electrical stimulation. The opioid antagonist naltrexone or placebo was administered between subjects to examine an analgesia due to anxiety-induced endorphinergic activity, while anxiety and focus of attention were manipulated within subjects. In accordance with previous research, pain ratings and skin conductance responses (SCRS) were not influenced by anxiety when focus of attention was controlled for. Altention towards pain led to an increase in subjective pain as opposed to distraction from pain. SCRs, howewer, were increased in the distraction conditions, probably due to heightened umexpectedness. Further, both high and low anxiety resulted in an analgesia compared to the pretest in the placebo condition, which was reversed by a low dose of naltrexone, but not by a high dose. Apart from possible agonist properties of high doses of naltrexone, this effect suggests an opioid-induced analgesia. It remains to be demonstrated whether this was due to endogenous opioids released during high anxiety.
\end{abstract}

\section{Introduction}

Several physiological and psychological mechanisms for the influence of anxiety on pain sensation have been proposed. Some of the hypothesized mechanisms suggest that anxiety increases pain, others imply a reduction in pain. Bolles \& Fanselow (1980) argued that fear and pain are mutually inhibiting states, fear having priority to promote fight or flight behaviour. In rats, analgesia can be produced by uncontrollable stress, a phenomenon usually attributed to the paininhibiting effect of endogenous opioids (e.g. Matzel \& Miller, 1987). Empirical evidence with humans comes from studies in which a stress-induced decrease in pain was reversed by an opioid antagonist (Willer \& Ernst, 1986; Pitman et al, 1990). Another physiological mechanism, contrasting with the role of endogenous opioids, is the stress-related release of epinephrine, which may sensitize or directly activate nociceptors (Chapman \& Turner, 1986).

In psychological accounts of the relation between anxiety and pain, the importance of the focus of anxiety is stressed: anxiety related to pain would increase subjective pain, whereas anxiety related to something else would result in a pain inhibition (Dougher, Goldstein \& Leight, 1987; Weisenberg et al., 1984; Al-Absi \& Rokke, 1991). According to attributional theory physiological arousal determines only the intensity of emotion, whereas the object to which it is attributed determines the type of emotion (Reisenzein, 1983). Anxiety focused on pain would increase pain because the arousal due to anxiety would be labeled 
as pain (Nisbett \& Schachter, 1966). In contrast, anxiety focused on a painirrelevant source may lead to a reduction in pain, because the arousal caused by the pain would be attributed to the source of anxiety. However, it has been demonstrated in several studies that these effects are mediated by attention (Arntz \& de Jong, 1993; Arntz, Dreessen \& Merckelbach, 1991; Arntz, Dreessen \& de Jong, 1994). Diverting attention away from pain stimuli resulted in lower pain ratings than directing attention towards them, irrespective of anxiety. The idea is that anxiety related to pain would lead to the allocation of attention to the pain stimulus, which would increase subjective pain, while the presence of other objects of fear would decrease pain by distracting attention from it. This would be consistent with reports of selective attention towards anxjety-related stimuli when people are in an anxious state (Eysenck, 1988).

Still, physiological factors may have a significant contribution. It is conceivable that an anxiety-induced release of endogenous opioids has a pain-decreasing effect that can be overruled by stronger attentional effects. Interestingly, it has been suggested that endorphinergic mechanisms may be involved in the regulation of selective attention (Rose \& Orlowski, 1983; Arnsten et al., 1983).

This study aimed at clarifying the role of attentional and endorphinergic mechanisms in the influence of anxiety on the sensation of acute pain stimuli. Pain was induced by electrical stimulation; anxiety was induced by exposing spider phobic subjects to a live spider. In a single-subject study (Thyer \&

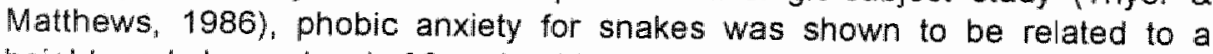
heightened plasma level of $\beta$-endorphins. In the present study , either placebo or the opioid antagonist naltrexone was administrated, which is known to reverse the effects of endorphinergic activity. Anxiety (high vs. low) and attention (towards pain vs. distracted from pain) were manipulated within subjects. If endogenous opioids indeed reduce pain during anxiety, one would expect that, with placebo but not with naltrexone, high anxiety has a pain-reducing effect as compared to low anxiety. Further, in accordance with previous studies, it was hypothesized that attention towards pain would result in more pain than distraction from pain, irrespective of anxiety condition.

\section{Method}

\section{Subjects}

Forty-five female spider phobics participated in the experiment in exchange for a free treatment of their phobia. Exclusion criteria were pregnancy, cardiovascular disease, liver or kidney dysfunction, use of opiate medication or psychotropics, addiction to alcohol or drugs, and psychopathology as indicated by a high score on the SCL-90 (above 149; Derogatis, Lipman \& Covi, 1973). Subjects' mean score on the Spider Phobia Questionnaire (SPQ; Klorman et al., 1974) was 22.3 ( $S D 4.1$ ). which is clearly in the phobic range. Ages ranged between 17 and 57
(mean age 28, SD $9.6 \mathrm{yr}$ ). 


\section{Materials}

Subjective pain, anxiety and attention paid to the stimuli were rated on 100 -mm Visual Analogue Scales (VASs). Scores were measured in millimeters. Fear of spiders was measured by the SPQ, other psychopathology by the SCL-90. A medium-sized live house spider was used to induce anxiety in the high anxiety conditions, and a video tape recording with an episode of L.A. Law was used in the low anxiety/distraction condition.

\section{Apparalus and physiological recording}

Apparatus was placed in an adjacent room. The pain stimulus consisted of electrical stimulation (20 msec rectangular pulses, with an interpulse time of 20 msec), produced by a Siemens Neuroton 627 . The stimulus was delivered at the subject's right ankle, which was cleaned with alcohol, via two $8 \mathrm{~mm} \mathrm{Ag}-\mathrm{AgCl}$ Beckman electrodes (distance $2.5 \mathrm{~cm}$ ) filled with Hewlett-Packard Redux Creme. It had a rising time of 2 sec to the preselected level, remained at that level for 2 $\mathrm{sec}_{\text {, }}$ and was returned to zero during $2 \mathrm{sec}$. The stimulation produces a painful. stinging sensation, which is unlike shocks caused by electric fencing, electric mains, etc. Skin Conductance Responses (SCRs) and Skin Conductance Level (SCL) were measured by a Beckman Skin Conductance Coupler (type 9844) using the method of constant voltage $(0.5 \mathrm{~V})$. Beckman electrodes $(8 \mathrm{~mm} \mathrm{Ag-}$ $\mathrm{AgCl})$ were filled with isotonic paste, and, after the skin was cleaned with distilled water, were attached to the medial phalanges of the second and third fingers of the left hand.

Table 1. The five within-subjects conditions, with anxiety (high vs. low), and attention (towards pain vs, distracted from pain) as factors.

\begin{tabular}{llll}
\hline & Low anxiety & High anxiety & \\
\hline Attention & LA/A & HA/A & HA/A \\
Distraction & LA/D & HA/D & \\
\hline
\end{tabular}

\section{Design}

A $3 \times 2 \times 2$ design was employed invalving pharmacological condition (placebo $25 \mathrm{mg}$ or $100 \mathrm{mg}$ of naltrexone) as between-subject factor, and anxiety (high vs. low) and attention (towards pain vs. distracted from pain) as within-subject factors. Both subject and experimenter were unaware of pharmacological condition. Fifteen subjects were randomly assigned to each condition. Each subject was exposed to the pain stimuli in the following conditions: low anxiefy/attention; low anxiety/distraction; high anxiety/distraction; and high anxiety/attention (see Table 1). Because the combination of inducing high anxiety by the presence of a spider and asking the subject to focus attention on the pain stimulus had proved difficult in earlier studies, two versions af the high anxietylatiention condition were used. The condition to use in the analysis was chosen a posteriori on the basis of manipulation checks. The order in which the 
five conditions were run was counterbalanced across subjects. The dependent variables in the analysis were pain VAS ratings and SCRs (square-root transformed and averaged in blocks of four trials per condition). SCL and anxiety and attention VAS ratings were used as a manipulation check. A pretest stimulation was given before pharmacological manipulation to check for initial group differences that may exist on these measures.

\section{Procedure}

After obtaining informed consent ${ }_{v}$ each subject was medically checked and the electrodes were fastened. Subsequently, the pain stimulation level was individually established by increasing the stimulation level in steps of $0.2 \mathrm{~mA}$ until pain threshold level (anchored at $25 \mathrm{~mm}$ on a $100 \mathrm{~mm}$ VAS, ranging from "no feeling at all" to "extremely painful"), and then increased further until a score of $70 \mathrm{~mm}$ on the VAS was reached. This procedure was repeated in order to enhance the reliability of the subjective pain level. The amperage corresponding to the $70 \mathrm{~mm}$ score of the second work-up procedure was maintained during the experiment. The mean pain threshold level was $1.6 \mathrm{~mA}$ (SD $0.7 \mathrm{~mA}$ ), the mean level used in the experiment was $3.5 \mathrm{~mA}$ (SD 1.3 mA). Next, a pretest was done, involving a series of four pain stimuli at the $70 \mathrm{~mm}$ level spread over $2 \mathrm{~min}$, with either 15,20 or 25 seconds as intertrial intervals. The subject was asked to concentrate as much as possible on the stimuli, which were not forewarned, and to describe them afterwards. After the last stimulus, subjeclive pain, anxiety and attention were scored on VASs. Subjects were informed that during the rest of the experiment the same four stimuli would be given, but that they were supposed to report any differences in subjective intensity. Either maltrexone (25 $\mathrm{mg}$ or $100 \mathrm{mg}$ ) or placebo was orally administrated, followed by a one hour waiting period (the peak plasma concentration of naltrexone occurs within one hour; ASHP, 1989). Questionnaires (SPQ, SCL-90) were completed. Subsequently, the series of four stimuli was given in five different conditions. Each next condition was started after a short waiting period, in which the 3 VASs were scored.

In the low anxietylattention condition (LA/A), the subject was asked to concentrate on the pain stimuli and to describe them afterwards. None of the stimuli was forewarned.

In the low anxiety/distraction condition (LA/D), the subject was asked to concentrate on the video and to try not to attend to the pain stimuli. The first stimulus was forewarned to prevent startling the subject.

In the first version of the high anxiely/attention condition (HA/A)), the subject was exposed to a spider in a glass jar and encouraged to do several exercises, such as pulling the spider towards her, touching it with a pencil, etc. Each stimulus was forewarned to enable the subject to stop and concentrate on the pain. After each stimulus the exposure was resumed until the next one was forewarned

In the second version of the high anxiety/attention condition $\left(H A / A^{2}\right)$, the subject was confronted with a spider in an opaque jar, which was shown and placed at a distance where the subject rated her fear about $50 \mathrm{~mm}$ on a VAS Again, the subject was asked to concentrate on the pain stimuli. None of the 
stimuli was forewarned.

In the high anxiety/distraction condition (HA/D), the subject was exposed to a spider in a glass jar and encouraged to do several exercises, such as pulling the spider towards her, touching it with a pencil, etc. The instruction was to concentrate on the spider and the exercises and to pay as little attention as possible to the pain stimuli. The first stimulus was forewarned to prevent stantling the subject.

After the last condition the subject was debriefed and thanked for participating.

\section{Results}

\section{Manipulation check}

There were no significant differences before administration between subjects receiving naltrexone or placebo on pretest pain $(F(2,42)=1.5)$ anxiety $(F(2,42)$ $=0.1)$ or attention ratings $(F(2,42)=0.5)$, nor on $\operatorname{SCR}(F(2,42)=2.3)$ or $S C L$ $(F(2,42)=1.5)$. There was, however, a marginal linear effect on the amperage level subjects reached $(t(42)=-1.72, p=0.09)$, with lower levels in the high dose condition. In order to distinguish effects of naltrexone condition from thase due to initiall differences, changes with respect to the pretest were used in the analyses of subjective ratings, SCL and SCR.

Three separate ANOVAs were performed to analyze the effects on anxiety ratings, attention ratings and $\mathrm{SCL}$. The following four contrasts were defined: Iow vs. high anxiety; attention vs. distraction; the interaction between these two; and $H A / A^{*}$ vs. HAIA ${ }^{2}$. To allow for directional interpretation, t-values are given when possible. The manipulation of anxiety proved to be very effective (see Table 2), the high anxiety conditions inducing much more anxiety than the low anxiety conditions $(t(42)=13.5, p<0.001)$, while SCL was also significantly increased $(t(42)=3.6, p<0.001)$. Overall, anxiety was rated lower in the attention than in the distraction conditions $(t(42)=3.0, p<0.005)$, but this was only due to $H A / A^{2}$. in which anxiety was rated lower than in $H A / A^{1}(t(42)=3.9, p<0.001)$. Attention on pain was rated much higher in the attention than in the distraction conditions $(t(42)=19.1, p<0.001)$. However, it was rated lower in the high anxiety than in the corresponding low anxiety conditions $(t(42)=-3.1, p<0.005)$, and lower in $H A / A^{1}$ than in $H A / A^{2}(t(42)=-3.6, p<0.001)$. Finally, pharmacological condition did not influence anxiety ratings $(F(2,42)=0.1$, n.s.), attention ratings $(F(2,42)=$ $1.5, \mathrm{n} . \mathrm{s}$.$) , or \mathrm{SCL}(\mathrm{F}(2,42)=1.2, \mathrm{n} . \mathrm{s}$. $)$.

Table 2. Means (SD) of the variables used as a manipulation check for each anxiety/attention condition, averaged over naltrexone conditions.

\begin{tabular}{llllll}
\hline & LA/A & LA/D & HA/A & HA/A & HAVD \\
\hline VAS Anxiety & $17(20)$ & $13(16)$ & $70(23)$ & $51(23)$ & $69(23)$ \\
SCL $(\mu$ Siemens) & $4.9(3.4)$ & $4.9(3.3)$ & $5.9(4.5)$ & $5.7(4.8)$ & $6.0(5.0)$ \\
VAS Attiention & $93(9)$ & $26(21)$ & $67(20)$ & $77(17)$ & $13(15)$ \\
\hline
\end{tabular}


On the basis of these manipulation checks it is not possible to select either HA/A" or $\mathrm{HA}_{\mathrm{A}} \mathrm{A}^{2}$ as the best high anxiety/attention condition. While $\mathrm{HA} / \mathrm{A}^{4}$ seems to have induced more anxiety. HA/A ${ }^{2}$ resulted in more attention towards the pain. Therefore it was decided to use all five conditions in the ANOVA, using the mean of the two HA/A conditions. Contrasts were defined for high vs. Iow anxiety, attention vs. distraction, and the interaction between these two. Because anxiety ratings in $H A / A^{1}$ were higher than in $H A / A^{2}$, another contrast was defined for high vs. low anxiety without $\mathrm{HAV}^{2}$, which was considered a more sensitive test for the presence of an anxiety effect.

Table 3. Means (SD) of the experimental variables for each anxiety/attention condition, averaged aver naltrexone conditions (SCR's are squaremroot transformed).

\begin{tabular}{llllll}
\hline & LANA & LAND & HA/A & HA/A & HAD \\
\hline VAS Pain & $57(23)$ & $42(23)$ & $42(24)$ & $60(24)$ & $33(27)$ \\
SCR(VuSiemens) & $0.49(0.35)$ & $0.56(0.38)$ & $0.49(0.23)$ & $0.44(0.27)$ & $0.50(0.31)$ \\
\hline
\end{tabular}

\section{Subjective pain}

As is shown in Figure 1 (and Table 3), attention directed towards pain led to higher pain VAS ratings than distraction from pain $(\mathrm{t}(42)=6.2, p<0.001)$. Whereas in previous research no effect of anxiety was seen, high anxiety resulted in less subjective pain than low anxiety $(t(42)=-2.3, p<0.05)$. There was no interaction between attention and anxiety $(t(42)=1.1, n$.s.). The effect of anxiety became more pronounced when $H A / A^{2}$, in which anxiety had not been induced satisfactorily, was excluded $(\mathrm{t}(42)=-5.8, p<0.001)$. These anxiety effects did not interact with naltrexone condition $(F(2,42)=0.6$, n.s.), meaning that they were probably not endorphinergic in nature. Rather, an overall effect of naltrexone was found (Table $4, F(2,42)=3.6, p<0.05$ ). This effect was quadratic $(t(42)=-2.6, p<0.05)$, not linear $(t(42)=-0.5$, n.s.), with higher pain ratings in the low dose condition than in either placebo or high dose condition.

However, because subjective attention towards pain was lower in the high anxiety conditions, any observed effect of anxiety condition may have been contaminated by differences in attention. Therefore, the same analysis was performed with attention rating as a covariate, thereby correcting for these differences. Now, the effect of naltrexone was only marginaliy significant $(F(2,41)$ $=2.9, p=0.06)$, but the quadratic effect was still apparent $(t(41)=-2.4, p<0.05)$. However, not only the attention effect $(t(41)=-1.1, n . s$.$) , but also the effect of$ anxiety entirely disappeared with attention rating as a covariate, either with or without $\mathrm{HAlA}^{2}(\mathrm{t}(41)=-0.5, \mathrm{n} . \mathrm{s} ; \mathrm{t}(41)=-1.1, \mathrm{n.s}$.). Thus, when the reduced amount of attention towards the pain in the high anxiety conditions was corrected for, no effect of anxiety on subjective pain ratings remained 


\section{Skin Conductance Response}

Unlike the increase in subjective pain, SCR's decreased with attention towards the pain (Table $3, t(42)=-2.1, p<0.05$ ). Anxiety did not lead to changes in SCR with all five conditions $(t(42)=-1.6, n$.s. $)$, nor with HAlA omitted $(t(42)=-0.97$, n.s.). There was, however, a marginal effect of naltrexone on $\operatorname{SCR}(F(2,42)=$ $3.21, p=0.05)$. Similar to the effect on subjective pain, this was quadratic (t) (42) $=-2.53, p<0.05)$, not linear $(t(42)=0.19$, n.s.), with highest SCRs in the low dose condition (Table 3).

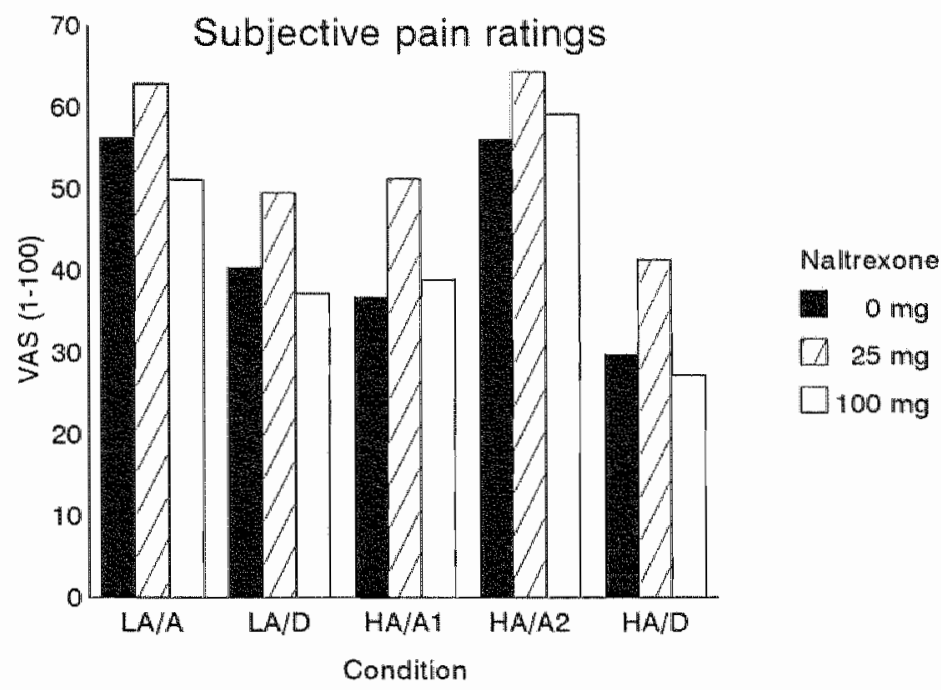

Figure 1 . Mean subjective pain ratings in the anxiety/attention conditions, displayed for each naltrexone condition.

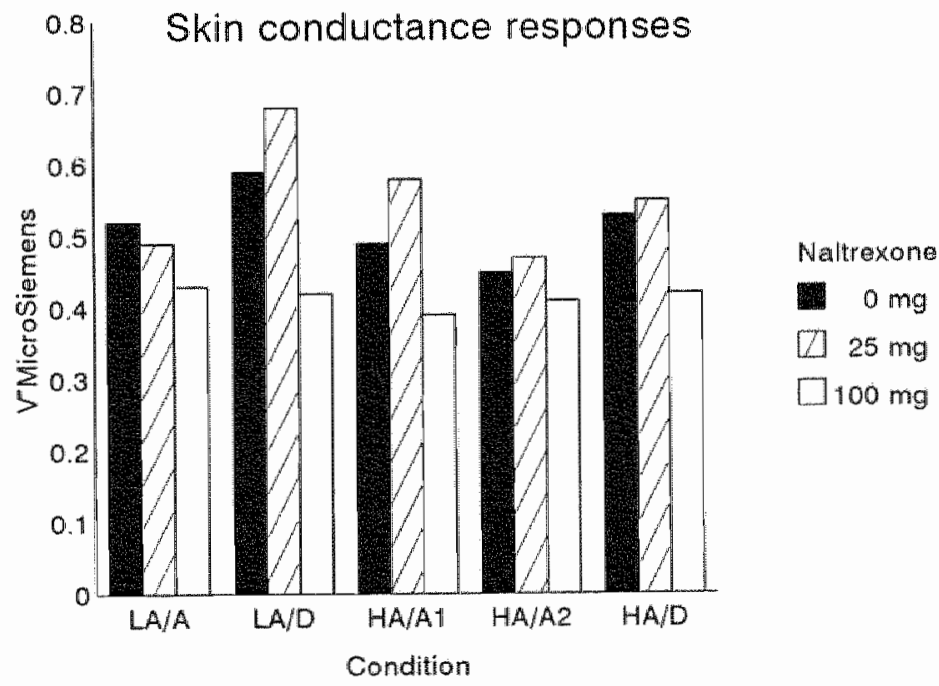

Figure 2. Mean skin conductance responses (square-root transformed and averaged for each block of 4 pain stimutity in the anxiety/attention conditions, displayed for each naltrexone condition. 


\section{Discussion}

The main conclusion to be drawn from the present data is that attention may have a primary role in the influence of anxiety on subjective and physiological pain responses. Although pain ratings were lower in the high anxiety conditions, this seems to be due to a failure to adequately focus attention on the pain stimulus when an object of fear is present, for the effect disappeared when differences in subjective attention were corrected for. Thus, the results strongly suggest that the anxiety-induced inhibition of pain is not a direct result of anxiety, but indirectly reflects attention effects. The fact that the instruction to focus attention on the pain was less effective (i.e. resulted in lower attention ratings), in the high anxiety conditions lends even further support to the idea that anxiety directs attention towards abjects of fear and could thereby influence pain perception.

There was, however, a discrepancy between subjective and physiological pain responses regarding the influence of distraction. Although it was related to a significant decrease in subjective pain, distraction from pain stimuli resulted in an increase in skin conductance response as compared to attention towards pain. This effect may be understood by considering SCRs not only as autonomic pain responses, but aiso as a reaction to the unexpectedness of stimuli. It could be argued that when attention is distracted the stimulus is simply less expected and may therefore evoke more autonomic arousal than when attention is directed towards the pain and the subject is waiting for the stimulus (cf. Arntz \& de Jong, 1993; Arntz, Dreessen \& de Jong, 1994).

With respect to the role of endorphins the results are less clear. Although anxiety did have an inhibiting effect on subjective pain, this appeared to be mediated by attention, rather than by release of endorphins, for it was not reversed by naltrexone. Instead, an effect of naltrexone itself was found, irrespective of anxiety condition, with a low dose of naltrexone increasing pain with respect to placebo and high dose.

However, the data do not rule out an interpretation in terms of an anxielyinduced release of endorphin. Since endorphinergic processes may be relatively slow (Matzel \& Miller, 1987), it is possible that endorphins released during high anxiety continued to act in low anxiety, the high and low anxiety conditions being mixed up. Thus, anxiety may still have caused the reduction in subjective pain and SCR with respect to the pretest which was observed in the placebo condition. Because it was absent in the low dose naltrexone condition, this analgesia appears to be reversed by naltrexone, and may therefore be endorphinergic in nature.

This interpretation cannot account for the observed dose effect, however: while a low dose of naltrexone resulted in higher pain ratings than placebo, a high dose of naltrexone did not. However, although naltrexone is usually described as a pure opioid antagonist (cf. ASHP, 1989), there is evidence that the intravenously administered version, naloxone, can act as an agonist (Lasagna, 1965; Levine, Gordon \& Fields, 1979; Levine \& Gordon, 1986).

Concluding, uncertainty still exists about the role of endorphins in the influence of anxiety on pain. Although a low dose of naitrexone seemed to reverse an 
analgesic effect, a high dose appeared to have agonist properties. It is possible that the type of pain stimulus used is not opioid-sensitive. However, studies in both rats (e.g. Fanselow, 1986) and humans (e.g. Willer \& Ernst, 1986) have shown an opioid-induced analgesia for electrical stimuli. The data plead for a follow-up study in which the high and low anxiety conditions are more separated in time and in which hormonal levels are determined. Nevertheless, in accordance with earlier studies (Arntz, Dreessen \& Merckelbach, 1991; Arntz \& de Jong, 1993; Arntz, Dreessen \& de Jong, 1994) these data suggest that not anxiety, but rather attention is one of the main factors influencing subjective and physiological pain responses during anxiety. 



\title{
3.3 No evidence for opioid-mediated analgesia induced by phobic fear
}

\author{
Summary
}

The influence of anxiety on acute pain sensation was investigated, stuidying the relative contribution of endogenous opioids and attentional mechanisms. Thirty-six spider phobics received mildly painful electrical stimulation, while anxiefy and focus of attention were manipulated within subjects. The opioid antagonist naloxone or placebo was administered between subjects to examine an analgesia owing to anxiety-induced endorphinerglc activity. In contrast to earlier findings ${ }_{\text {" }}$ attention towards pain failed to increase pain as opposed to distraction from pain, probably owing to a less effective attention manipulation. Furthermore "despite high levels of anxiety, subjective pain ratings were not influenced by anxiety, although heart rate responses were slightly inhibited. Accordingly, there was no increase in subjective or physiological pain responses as a result of naloxone, nor did $\beta$-endorphin plasma levels rise during anxiety. The results suggest that phobic anxiety does not induce an opiold-mediated analgesia. Curiously, naloxone itself effected a dose-dependent analgesia compared to placebo during both high and low anxiety, which is compatible with the assumption of agonist properties of naloxone in the absence of opioid activity.

\section{Introduction}

Anxiety is often believed to influence the perception of pain. However, the nature and the mechanisms of influence are unclear. Some claim that anxiety may enhance pain perception because epinephrine released during anxiety sensitizes nociceptors (Chapman \& Turner, 1986), leading to the use of relaxation techniques in pain control. In contrast, Boiles \& Fanselow (1980) argued that fear inhibits pain to promote defensive behaviour. They illustrate this by the phenomenon of stress-induced analgesia in rats, which is attributed to the analgesic effect of endogenous opioids. Although studies with humans are scarce, some evidence has been found for stress-induced decreases in pain mediated by endogenous opioids (Willer \& Ernst, 1986; Pitman et al, 1990). Also, cardiac rates during pain proved to be attenuated by anxiety (Carter, MCNeill \& Reed, 1991).

It is possible that, while endocrine responses to stress do significantly affect pain perception in animals, higher cognitive and emotional functions are more important in humans. Psychological theories stress the importance of the focus of anxiety: pain responses would be increased only when pain itself is the object of fear (Weisenberg et al., 1984; Dougher, Goldstein \& Leight, 1987; Al-Absi \& Rokke, 1991). A possible explanation is based on attributional theory, stating that physiological arousal determines the intensity of emotion, while the object to 
which it is attributed determines the type of emotion (Reisenzein, 1983). Anxiety related to the object of pain would increase pain because arousal owing to anxiety will be labeled as pain (Nisbett \& Schachter, 1966). Instead, the presence of another object of fear may reduce pain, because arousal will be attributed to the source of anxiety. Several studies have indicated, however, that the effect of focus of anxiety is mediated by attention (Arntz, Dreessen \& Merckelbach, 1991; Arntz \& de Jong, 1993; Arntz, Dreessen \& de Jong, 1994). When anxiety and focus of attention were independently manipulated, attention towards pain led to stronger pain sensations than attention distracted from pain, irrespective of anxiety.

In another study (Janssen \& Arntz, 1996), the relative contribution of attentional focus and endogenous opioids was studied. Anxiety was induced by confronting spider phobics with a spider, attention was manipulated by instructions. The release of endorphins may be promoted by phobic stimuli (Thyer \& Matthews, 1986) and may even be involved in approach behaviour during exposure (Merluzzi et al., 1991; Arntz, Merckelbach \& de Jong, 1993). Either placebo or the opioid antagonist naltrexone, known to reverse the effects of endorphinergic activity, was administered. Again, anxiety did not affect pain when focus of attention was controlled for, nor did naltrexone show any selective effects. However, high and low anxiety conditions succeeded each other quickly in time. Since endorphinergic processes may be relatively slow (Matzel \& Miller. 1987). opioid-mediated analgesia may not have been restricted to the high anxiety conditions. Indeed, low dose naltrexone showed an overall painincreasing effect compared to placebo. Unexpectedly, high dose naltrexone did not show this effect, suggesting that naltrexone is not a pure antagonist.

In the present study, we further studied endorphinergic influences on pain, this time with high and low anxiety conditions separated in time. Instead of naltrexone, the more commonly used naloxone was chosen as an opioil antagonist. Since the release of catecholamines may be stimulated by naloxone during extreme sympathetic activation (ASHP, 1989), blood plasma levels were determined to control for any influence of (nor)epinephrine on pain. Also, $\beta$ endorphin plasma levels were determined, viewed as indicators for central endorphinergic activity (Cohen, Pickar \& Dubois, 1983). It was hypothesized that, with placebo but not with naloxone, high anxiety would have a pain-reducing effect compared to low anxiety. Furthermore, an effect of focus of attention was expected, with highest subjective pain ratings in the attention condition and lowest in the distraction condition. An attentional ambiguous condition was added, since, in previous studies, endorphinergic influences on pain may have been overruled by attentional focus as the result of the strong manipulation of attention versus distraction.

\section{Method}

\section{Subjects}

Thirty-six female spider phobics participated in two experimental sessions on separate days in exchange for a free treatment of their phobia. Exclusion criteria were pregnancy, liver or kidney dysfunction, cardiovascular disease, use of opiate 
medication or psychotropics, addiction to alcohol or drugs, and other psychopathology as indicated by a high score on the SCL-90 (above 149 ). Subjects" mean score on the Spider Phobia Questionnaire was 23.5 (SD 3.6), which is clearly in the phobic range (cf. Arntz et al.. 1993b). Mean blood phobia on the Fear Questionnaire was low (8.1, SD 4.7). Ages ranged between 17 and 42 (mean age $29, \mathrm{SD} 6.9 \mathrm{yr}$ ).

\section{Malenals}

Subjective pain, anxiety and amount of attention paid to the pain stimuli were rated on 100-mm Visual Analogue Scales (VASs). Scores were measured in millimeters. The Fear Questionnaire (FQ; Marks \& Mathews, 1979) was used to assess the extent of subjects' fear of blood and injections. Fear of spiders was measured by the Spider Phobia Questionnaire (SPQ; Klorman et al. , 1974), other psychopathology by the SCL-90 (Derogatis, Lipman \& Covi, 1974). In the high anxiety session a Behavioural Approach Test (BAT; cf. Arntz, Merckelbach \& de Jong, 1993) involving a medium-sized live house spider was used to induce anxiety, as well as a video tape recording of a spider exposure treatment session. In the low anxiety session a video tape recording of a documentary about a gllass factory and an episode of L.A. Law were shown.

\section{Apparatus and physiological recording}

Apparatus was placed in an adjacent room. The pain stimulus consisted of electrical stimulation (20 msec rectangular pulses of alternating current). It was delivered at the left ankle, which was cleaned with alcohol, wia two $8 \mathrm{~mm} \mathrm{Ag-}$ AgCl SensorMedics electrodes (distance $2.5 \mathrm{~cm}$ ) filled with Hewlett-Packard Redux Creme. It had a rising time of 0.5 sec to the preselected level, remained constant for $2 \mathrm{sec}$, and returned to zero during $0.5 \mathrm{sec}$. The stimulation produces a painful, stinging sensation, which is unlike shocks caused by electric fencing. electric mains, etc.

Heart rate was measured by three $8 \mathrm{~mm} \mathrm{Ag-AgCl}$ SensorMedics electrodes, filled with Redux Creme and connected to the registration apparatus (Picker Schwarzer ED 14). After the skin was cleaned with alcohol, the electrodes were placed just above the sternum, at the left side of the chest, and at the left shoulder as a reference. Interbeat intervals were determined, and transformed to heart rate in beats per minute on a second-to-second basis. Heart rate response was defined as the mean change in heart rate (in beats per minute) during the 20 seconds after the onset of the stimulus, using the mean heart rate during the 5 sec prior to onset as reference.

\section{Elood samples and pharmacological manipulation}

For blood taking and injection of naloxone or placebo a catheter (Venflon $1.0 / 32$ $\mathrm{mm}$ ) was inserted in the subject"s forearm or elbow. This was done at least 30 minutes before the first pain ratings. Immediately after the pretest saline placebo, $2 \mathrm{mg}$ or $4 \mathrm{mg}$ of naloxone hydrochloride $(0.4 \mathrm{mg} / \mathrm{fm})$ was administered intravenously. To ensure that naloxone in plasma remained more or less stable during the experiment (plasma half-life is 60-90 minutes), booster doses were given before each condition. which was after 20 minutes (placebo, $0.4 \mathrm{mg}$ or 0.8 
mg), and after 30 and 40 minutes (placebo, $0.2 \mathrm{mg}$ or $0.4 \mathrm{mg}$ ). Immediately after each condition, $5 \mathrm{~mL}$ blood samples were collected for catecholamine analysis in tubes on ice containing glutathione and heparin. After the last condition, an additional $5 \mathrm{~mL}$ blood was sampled in heparin-containing lubes on ice for the analysis of plasma p-endorphin. All samples were spun down at 3000 rpm for 10 mirutes at $0^{\circ} \mathrm{C}$ and stored at $-40^{\circ} \mathrm{C}$. Plasma catecholamines were analyzed by high-performance liquid chromatography, $\beta$-endorphins by radioimmunoassay.

Table 1. The within-subjects conditions, with anxiety (low vs. high), and attention (attention on pain, ambiguous, distraction from pain) as factors.

\begin{tabular}{lllll}
\hline & & & \multicolumn{2}{c}{ Experimental phase } \\
\hline & & Attention & Ambiguous & Distraction \\
\hline Low Anxiety & Neutral video & LA/Att & LA/Amb & LAVDis \\
High Anxiety & Spider exposure & HAVAtt & HA/Amb & HAVDis \\
\hline
\end{tabular}

Design

A $3 \times 2 \times 2 \times 3$ design was employed involving pharmacological condition (placebo, low or high dose of naloxone) and session orcer (high or low anxiety first) as between-subject factors, and anxiety (high vs low) and attention (attention on pain, distraction from pain, and an ambiguous condition) as within-subject factors (see Table 1). High and low anxiety were induced on separate days, about a week apart at the same hour of day, while attention was manipulated within each session. An induction was included in each session before the experimental phase, presuming that endorphinergic processes may be relatively slow. Twelve subjects were randomly assigned to each pharmacological condition, of which both subject and experimenter were unaware. Session order was counterbalanced across subjects, as was the order in which attention conditions were run. Dependent variables were pain VAS ratings and heart rate responses. Anxiety and attention VAS ratings and endocrine plasma levels were used as manipulation check. In both sessions, a pretest stimulation was given betore pharmacological manipulation.

\section{Procedure}

Each subject participated in a high and a low anxiety session, the order of which they knew beforehand. In the first session they were medically checked by a doctor and informed about the medical procedures. After inserting the catheter and fastening the electrodes, the pain stimulation level was individually established. The level was increased in steps of $0.2 \mathrm{~mA}$ until pain threshold level (anchored at $25 \mathrm{~mm}$ on a $100 \mathrm{~mm}$ VAS, ranging from "no feeling at all" to 'extremely painful'), and then increased until reaching $70 \mathrm{~mm}$ on the VAS. This level was maintained during the experiment. In the second session the level was increased in small steps again until the same $\mathrm{mA}$ level was reached. However, 
five subjects (four in the LA/HA order, and one in the HA/LA order) did not tolerate their prior $\mathrm{mA}$. level in the second session. Therefore, a linear correction for the slightly lower $\mathrm{mA}$ levels was done on their pain ratings. Mean threshold level was $2.0 \mathrm{~mA}$ (SD 1.1), mean level used in the experiment was $3.4 \mathrm{~mA}$ (SD 1.2). A pretest was done, involving a series of four pain stimuli spread over 2 minn $_{n}$ along with the instruction to concentrate on the stimuli. The first one was announced. Afterwards the 3 VASs were scored. Subjects were told that during the rest of the experiment the stimuli would be of the same intensity, but that they were supposed to rate their experience of the pain stimulation. Subsequently either saline placebo, low or high dose naloxone were administered. After $3 \mathrm{~min}$ (the onset of action is 1-2 min) the induction of low or high anxiety was started.

Induction phase: In the low anxiety session a 6 min neutral video was shown, followed by the series of pain stimuli. In the high anxiety session a Behavioral Approach Test (BAT) was used, involving a spider in a glass jar placed at $1.5 \mathrm{~m}$ from the subject in a transparent container on a small plateau. The subject was asked to perform several steps like pulling the plateau towards her, opening the jar, touching the spider, etc., verbally rating the percentage of fear on a scale ranging from completely relaxed to extreme panic. Then the jar was put at a distance corresponding to a fear rating of 60 percent, followed by the series of four pain stimuli 2 min later. After stimulation the 3 VASs were scored.

Immediately after the induction phase the experimental phase started. Both high and low anxiety session comprised three conditions with different attention instructions. Before each condition a booster dose of naloxone or placebo was given, afterwards blood was sampled. Each condition lasted $4 \mathrm{~min}$, the four pain stimuli spread over the last $2 \mathrm{~min}$. The first one was always announced, with a short repetition of the instruction; after the last one the 3 VASs were scored.

Attention condition: In the low anxiety session, the instruction was to concentrate as much as possible on the pain stimulus and describe it afterwards. In the high anxiety session the instruction was similar, although an opaque jar containing the spider in the jar was included at a distance where the subject rated fear about 60 percent. If fear was not high enough with the spider invisible, the opaque jar was removed.

Ambiguous condition: The subject was asked to watch a video tape recording, either showing an episode of L.A.Law (low anxiety) or a spider exposure session (high anxiety). However, the subject was instructed to concentrate as much as possible on the pain stimulus when it was given, and describe it afterwards. The high anxiety session included the opaque jar with the spider at $1.5 \mathrm{~m}$ distance.

Distraction condition: the subject was also asked to watch a video tape recording, either showing an episode of L.A.Law (low anxiety) or a spider exposure session (high anxiety), but now with the instruction to concentrate as much as possible on the video and to ignore the pain stimulus. The high anxiety session included the opaque jar with the spider at $1.5 \mathrm{~m}$ distance. 


\section{Results}

\section{Manipulation check}

Threshold, selected $\mathrm{mA}$ level and pretest pain ratings did not differ significantly between naloxone groups or session order groups, nor were pretest pain ratings affected by anticipation of high vs. low anxiety $(F(1,30)=0.3$, n.s.).

Anxiety ratings were significantly increased in the high vs. the low anxiety condition (see Table 2), both in the induction phase $\{F(1,30)=153.6, \rho<0.001)$ and in the experimental phase $(F(1,30)=161.4, p<0.001)$. Pharmacological manipulation or attention condition did not influence anxiety ratings.

As shown in Table 2, attention ratings were influenced by the manipulation of attention $(F(2,60)=113.7, p<0.001)$, with most attention on pain in the attention, least in the distraction condition, with the ambiguous condition in between (linear $t(60)=-12.4, p<0.001)$. However, during high anxiety less attention was directed towards pain than during low anxiety $(F(1,30)=29.9, p<0.001)$, with an interaction between anxiety and attention condition $(F(2,60)=8.3, p<0.001)$, owing to a less effective attention manipulation in the high anxiety condition.

Table 2. Means (SD) of subjective variables NAS) and heart rate response (beats per minute), averaged over naloxone conditions.

\begin{tabular}{llllll}
\hline Session & & Ind & Att & Amb & Dis \\
\hline \multirow{3}{*}{ Low Anxiety } & Anxiety & $14(18)$ & $12(14)$ & $10(11)$ & $10(14)$ \\
& Attention & $73(22)$ & $82(19)$ & $65(19)$ & $26(19)$ \\
& Pain & $54(23)$ & $51(20)$ & $49(22)$ & $45(22)$ \\
& HRR(bpm) & $1.5(2.7)$ & $1.2(2.4)$ & $1.3(2.2)$ & $0.8(2.0)$ \\
\hline \multirow{3}{*}{ High Anxiety } & Anxiety & $70(19)$ & $54(20)$ & $65(23)$ & $64(26)$ \\
& Attention & $44(24)$ & $58(23)$ & $52(22)$ & $22(21)$ \\
& Pain & $56(25)$ & $52(25)$ & $46(25)$ & $53(29)$ \\
& HRR(bpm) & $0.9(2.3)$ & $0.4(2.1)$ & $0.3(3.3)$ & $0.3(3.2)$ \\
\hline
\end{tabular}

\section{Subjective pain ratings}

Pain ratings were analyzed by a $3 \times 2 \times 2 \times 3$ analysis of variance (naloxone $x$ session order $x$ anxiety $x$ attention), while the induction phase was analyzed separately in a $3 \times 2 \times 2$ analysis (naloxone $x$ session order $x$ anxiety). There was no effect of anxiety session (see Table 2), neither in the induction $(F(1,30)$ $=0.2$, n.s.) nor in the experimental phase $(F(1,30)=0.9$ n.s.). Accordingly, there was no significant interaction between anxiety session and naloxone condition in the induction $(t(30)=1.6, n . s$. $)$ or experimental phase $(t(30)=1.4, n . s$.). Instead, in the experimental phase, naloxone showed a marginally significant paininhibiting effect irrespective of anxiety session (linear dose trend, $t(30)=-1.8, p$ $=0.09$ ). When corrected for initial differences by subtracting each session's 
pretest pain rating this was more pronounced, in the induction $(t(29)=-2.6$. $p<$ $0.05)$ and in the experimental phase $(t(29)=-3.6, p<0.001)$, while an anxiety effect was still absent (see Figure 1). Further, an interaction between naloxone and attention condition $(t(30)=-2.3, p<0.05)$ indicated that this effect was largest in the ambiguous condition, providing some support for the assumption that physiological influences on pain are only apparent in the absence of a strong attention manipulation.

Attention condition did not significantly affect pain ratings $(F(2,60)=1.2$, n.s.), even when attention and distraction condition were contrasted $(t(30)=0.3, n . s$.). In the low anxiety condition, however, pain ratings were slightly reduced by distraction, leading to a marginally significant interaction between attention and anxiety condition $(t(30)=-1.7, p=0.10)$.

\section{Heart rate responses}

Within each anxiety session (including the induction), healt rate response was used as dependent variable, with anxiety session and naloxone condition as factors. Mean heart rate during 5 sec before each stimulation was significantly increased in the anxiety session (78.0 vs. $71.9 \mathrm{bpm} ; \mathbb{F}(1,33)=24,6, p<0.001)$, but showed no effect of naloxone condition $(\mathbb{F}(2,33)=0.05$, n.s.). There were no initial differences between naloxone groups on heart rate response $(F(2,66)=$ 1.07 , n.s.). Anxiety session showed a marginally significant effect, with lower responses for high anxiety $(F(1,33)=3.17, p=0.08)$. However, neither the interaction between anxiety and naloxone condition (linear dose trend, $t(33)=$ 1.37 , n.s.), nor the main effect of naloxone condition (linear dose trend, $t(33)=$ $0.36, n$.s.) were significant. A separate analysis showed that attention condition did not affect heart rate response $(F(2,66)=0.25$, n.s. $)$.

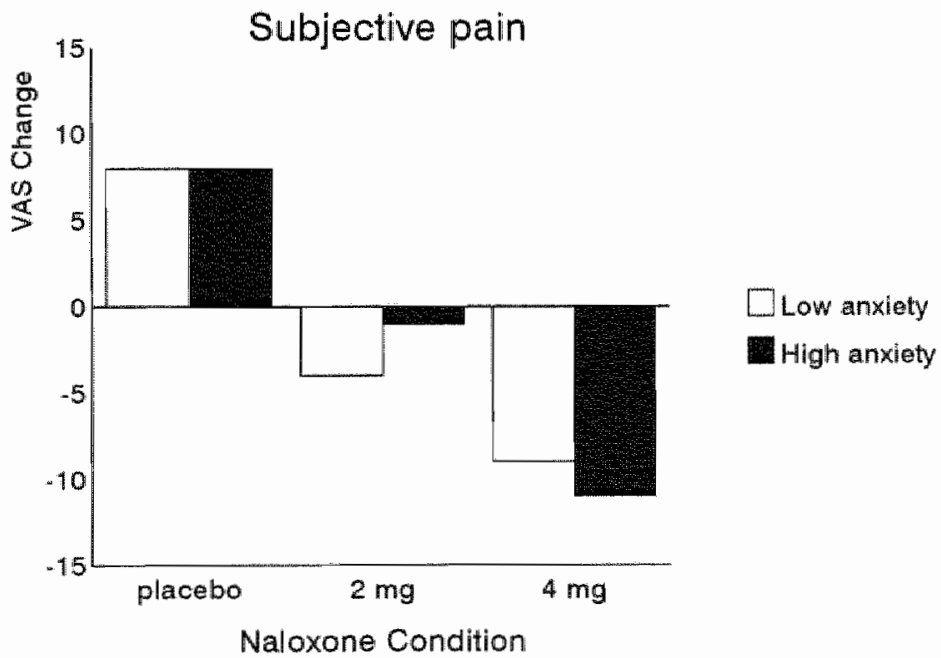

Figure 1. Mean change in subjective pain with respect to the pretest in each nalox one condition, displayed for low and high anxiety. 


\section{Blood samples}

Since no effect of attention condition was found on pain responses, it was considered redundant to do the (expensive) catecholamine analysis for all attention conditions. Therefore, from each session, catecholamine levels were only determined for the second attention condition, resulting in an equal distribution because the order of attention conditions was counterbalanced. Owing to sampling failures, two values for catecholamines and three for $\beta$-endorphins were missing. The effect of anxiety on epinephrine was marginally significant $(F(1,28)=3.0, p=0.10)$, as was the interaction between anxiety and naloxone condition on norepinephrine (linear dose trend, $t(28)=-1.9, p=0.06$ ). Although naloxone slightly increased (nor)epinephrine plasma levels during high anxiety (see Table 3), this could not have accounted for the pain-inhibiting effect of naloxone, for if any effect of catecholamines were expected, it would be painincreasing (Chapman \& Turner, 1986). B-endorphin seemed slightly increased during anxiety $(F(1,27)=2.9, p=0.10)$, but this rise was only shown by two outliers receiving low dose naloxone, resulting in an interaction between anxiety and naloxorie condition $(F(2,27)=3.9, p<0.05)$.

Table 3. Plasma endocrine levels (SD) in the low and high anxiety session.

\begin{tabular}{lllll}
\hline Plasma lewel $(\mathrm{pg} / \mathrm{ml})$ & & Nal $0 \mathrm{mg}$ & Nal 2 mg & Nal 4 mg \\
\hline \multirow{3}{*}{ Low Anxiety } & B-endorphin & $22.5(4.9)$ & $24.9(14.5)$ & $22.5(4.4)$ \\
& Epinephrine & $30.7(19.0)$ & $29.9(17.8)$ & $27.7(9.0)$ \\
& Norepinephrine & $240.9(79.3)$ & $190.8(43.7)$ & $194.2(50.9)$ \\
\hline \multirow{3}{*}{ High Anxiety } & B-endorphin & $22.1(4.2)$ & $30.1(18.7)$ & $21.4(6.2)$ \\
& Epinephrine & $31.5(13.4)$ & $36.7(20.1)$ & $33.1(19.5)$ \\
& Norepinephrine & $216.6(41.5)$ & $206.3(52.1)$ & $212.3(37.1)$ \\
\hline
\end{tabular}

\section{Discussion}

This study suggests that anxiety "even when pathological, does not influence subjective pain through endogenous opioids. The absence of an anxiely-induced analgesia was parallelled by the absence of a pain increase by naloxone during high anxiety. Only heart rate responses were slightly inhibited by anxiety (reminiscent of Carter, McNeill \& Reed, 1991), but this effect did not reach significance and may be due to ceiling effects. Instead, naloxone effected a dosedependent analgesia, irrespective of anxiety condition. This is compatible with evidence that naloxone may have analgesic properties (Buchsbaum, Davis \& Bunney, 1977; Levine, Gordon \& Fields, 1979; Stacher et al., 1988). However, these same doses did increase pain following stress in other studies (Willer \& Albe-Fessard, 1980; Pitman et al., 1990; Janssen \& Arntz, submitted), suggesting 
that naloxone only inhibits pain in the absence of increased opioid activity. Indeed, $\beta$-endorphin plasma levels did not rise during anxiety.

The second hypothesis, predicting an effect of attentional focus, was also contradicted, although there was a small attention effect in the low anxiety session. This result is in contrast with many previous studies (e.g. Amtz, Dreessen \& Merckelbach, 1991; Arntz, Dreessen \& de Jong 1994; Arntz \& de Jong, 1993; Janssen \& Arntz, 1996) demonstrating a clear pain inhibition when attention was distracted from pain. Perhaps the medical interventions prevented a successful manipulation of attention, especially in the spider's presence, where attention ratings showed that subjects had difficulty following attention instructions. Another explanation involves stimulus characteristics: the stimulus reached its maximum within 1 sec, against $2 \mathrm{sec}$ in previous studies. We tested the impact of this difference in a study with 40 students (unpublished), of which half received the sudden stimulus and half the slower one. The group with the slower stimulus showed a significant reduction in pain during distraction $(F(1,18)=11.5$, $p<0.005)$, whille the group with the sudden stimulus did not $(\mathbb{F}(1,18)=2.37$, n.s.). It could be argued that sudden stimuli automatically attract attention, irrespective of manipulation. Although attention ratings in the present study were affected by instructions, during the stimuli itself attention may have been focused primarilly on the pain.

Why do so many animal studies demonstrate a stress-induced analgesia while such effects in humans are hardly ever found? There is evidence from studies with rats that uncontrollability is a necessary condition for stress-induced analgesia (Maier ef al., 1983). Henry (1990) claimed that different kinds of stress may lead to different endocrine responses, catecholamine release prevailing when fight or flight behaviour is required, whereas the adrenal cortical axis is activaled when the subject is helpless and not in control of the situation. While animal phobias have led to some of the strongest emotional reactions in human subjects under laboratory conditions, they can be accompanied by weak or even absent endocrine responses (Curtis \& Glitz, 1988). Although the spider phobics in this study were very anxious, the situation may have been perceived as relatively controllable. More extreme anxiety or uncontrollability is for ethical reasons, hard to reach in the human laboratory. Indeed, in a study with subjects exposing themselves to the more real-ifie stress situation of a first time parachute jump, an opioid-mediated analgesia was found (Janssen \& Arntz, submitted). This supports the idea that not just anxiety, but also a certain amount of uncontrollability is a precondition for the release of endogenous oploids. 



\section{No interactive effects of naltrexone and benzodiazepines on pain during phobic fear}

\section{Summary}

Animal research has shown that anxiety may imhibit pain through the release of endogenous opioids. On the other hand, anxiety is often believed to exacerbate pain in clinical situations, and anxiety reduction has been shown to attenuate the affective component of pain. In the present study phobic anxiety was induced by confronting forty-eight spider phobic subjects with a spider, after which they received two mildy painful electrical stimuli at two different current levels. The benzodiazepine alprazolam ( $1 \mathrm{mg}$ ) was administered to investigate the influence on pain of a reduction in anxiety. while the role of endogenous opioids was studied by administering the opioid antagonist naltrexone $(50 \mathrm{mg})$. Alprazolam resulted in lower anxiety and pain ratings during pain stimulation, supporting the idea that (presumably pain-related) anxiety may increase the experience of pain. Naltrexone did not influence pain and anxiety ratings, nor was there a significant interaction between the two pharmacological manipulations. These findings confirm previous evidence that phobic fear does not necessarily induce an endogenous opioid-mediated analgesia.

\section{Introduction}

Animal research has shown that uncontrollable stress or fear may induce analgesia through the release of endogenous opioids (e.g. Maier et al., 1983). Some studies have demonstrated similar stress-induced analgesic effects in humans, reversable by an opioid antagonist (Willer \& Ernst, 1986; Pitman et al., 1990; Janssen \& Arntz, submitted). It has been argued that endogenous opioids released during fear inhibit pain in order to promote fight or flight behaviour (Bolles \& Fanselow, 1980; Fanselow, 1991). Assuming that fear inhibits pain through the release of endogenous opioids, ways of reducing fear would be expected to reverse these analgesic effects. This is confirmed by findings that benzodiazepines, known for their sedating and anxiolytic effects, attenuated opioid analgesia in animals (Fanselow \& Helmstetter, 1988; Rovati et al, 1990). There is evidence that benzodiazepines do not influence analgesic effects after one or five shocks, but do attenuate analgesia in situations with a large enough number of inescapable shocks to produce learned helplessness (Maier, 1990). This may explain the findings of other animal studies in which benzodiazepines failed to attenuate opioid analgesia (Hayes et al., 1978; Chance et al., 1979; Kinscheck, Watkins \& Mayer, 1984). In humans, benzodiazepines have been shown to partly inhibit stress-induced analgesic effects that were reversed by an 
opioid antagonist (Willer \& Ernst, 1986), and to block stress-induced increases in endocrine markers of adrenal cortical axis activity, particularly ACTH and cortisol (Roy-Byrne, Risch \& Uhde, 1988). Recently, Gear et al. (1997) found that the benzodiazepine antagonist flumazenil enhances morphine analgesia in humans. The above findings suggest that, through reducing anxiety, benzodiazepines attenuate opioid analgesic effects of anxiety, compatible with the idea of Fanselow (1991) that the emotional component of fear is the most important mediator of endogenous opioid-mediated analgesia.

In contrast, the increased arousal, sympathetic outflow or muscle tension associated with anxiety are often believed to enhance pain experience (Chapman \& Turner, 1986; Dougher, Goldstein \& Leight, 1987). This idea has led to the administration of benzodiazepines to minimize pain in clinical practice (Chapman \& Feather, 1973; Singh et al., 1981). Benzodiazepines do seem to ameliorate the affective component of acute pain, possibly as a result of their anxiolytic action (Dellemijn \& Fields, 1994). They are known to activate $\mathrm{GABA}_{\mathrm{A}}$ receptors, although this does not seem to account for all of their actions (Sanger, 1985). Interestingly, benzodiazepines have been argued to exert part of their action through endogenous opioid activity, since some effects have been shown reversable by an opioid antagonist (Dingledine, 1978; Tripp \& McNaughton, 1992).

The present study investigates the influence of anxiety on the experience of an acute pain stimulus, and the mediating role of endogenous opioids. Spider phobic subjects were confronted with a spider, which may be viewed as an ethical way to induce a state of real anxiety in the human laboratory. In a singlesubject study (Thyer \& Matthews, 1986), phobic anxiety for snakes was shown to be related to a heightened plasma level of $\beta$-endorphins. Furthermore, effects of an opioid antagonist were found in spider phobics doing a spider approach test, on behavioural measures (Merluzzi et al., 1991; Arntz, Merckelbach \& de Jong, 1993), and on pain experience (Janssen \& Arntz, 1996). However, in a study with spider phobics in which both pain sensitivity and $\beta$-endorphin plasma levels were measured, no evidence for endogenous opioid effects was found (Janssen \& Arntz, 1997). This was attributed to the relative controllability of the confrontation with the spider, based on evidence that analgesic effects may be restricted to situations in which threat is uncontrollable or inescapable (Maier et al. 1983). Therefore "the present study comprises a "flooding"-like confrontation with a spider, which is far less controllable for the subject than a voluntary approach test. Also, since the release of endogenous opioids may be a relatively slow process (Matzel \& Miller, 1987; Lewis, Cannon \& Liebeskind, 1980), subjects were repeatedly confronted with the spider before pain sensitivity was measured. Endogenous opioid activity and anxiety were experimentally varied by pharmacological manipulation. To detect analgesic effects due to endogenous opioids, either placebo or the opioid antagonist naltrexone was administered, naltrexone being expected to reverse endogenous opioid effects seen after placebo administration. Furthermore, half of the subjects in each group were given the benzodiazepine alprazolam to test the effect of a reduction in anxiety on pain sensitivity. Two contrasting hypotheses were tested

1) If anxiety reduces pain through the release of endogenous opioids (Fanselow, 
1991), reducing anxiety by alprazolam, as well as blocking endogenous opioids by means of an opioid antagonist, would be expected to reverse this pain reduction. An interaction between alprazolam (yes/no) and naltrexone (yes/no) would be expected, with naltrexone andior alprazolam leading to an increase in pan compared to the placebo alone condition.

II) If, on the other hand, anxiety increases pain through enhanced arousal or sympathetic outflow, alprazolam would be expected to reduce pain compared to no alprazolam (lla). Furthermore, if effects of alprazolam are mediated by opioid mechanisms, which is suggested by findings that $G A B A$-effects were reversed by an opioid antagonist (Dingledine, Iversen \& Breuker, 1978; Tripp \& McNaughton, 1992), naltrexone should block this pain reduction by alprazolam. In that case, an interaction between naltrexone (yes/no) and alprazolam (yes/no) would be expected, with alprazolam alone leading to a decrease in pain compared to the placebo alone condition, but alprazolam resulting in similar pain levels as no alprazolam when administered together with naltrexone (lib)

\section{Method}

\section{Subjects}

Forty-eight female spider phobics participated in the experiment in exchange for a free treatment of their phobia. Exclusion criteria were pregnancy, heart, liver or kidney dysfunction, use of opiate medication and addiction to alcohol or drugs, and known hypersensitivity to benzodiazepines. Subjects' mean score on the Spider Phobia Questionnaire (SPQ; Klorman et al. 1974) was 23.2 (SD 2.B), which is clearly in the phobic range (Arntz et al., 1993b). Ages ranged between 16 and 60 (mean age 29, SD 8.4 yr). Subjects signed an informed consent about the procedure, which was approved by our Medical Ethics Committee.

\section{Materials}

Subjective pain, anxiety and positive excitement during stimulation were rated on $100-\mathrm{mm}$ Visulal Analogue Scales (VASs) ranging from "not at all..." to "extremely...". Scores were measured in $\mathrm{mm}$. A behavioural approach test (BAT, cf. Arntz, Merckelbach \& de Jong, 1993) with a medium-sized live house spider and a "flooding"-like confrontation with a large live house spider (7 cm) were used repeatedly to induce anxiety and to promote the release of endogenous opioids.

\section{Apparatus and physiological recording}

Apparatus was placed in an adjacent room. The pain stimulus consisted of electrical stimulation (20-ms rectangular pulses, with an interpulse time of $20 \mathrm{~ms}$ ). produced by an Eltron D (Enraf Nonius) stimulator. Two stimulations of $3 \mathrm{~s}$ were given, at two levels of stimulation: low current $(2.5 \mathrm{~mA})$ and high current $(5 \mathrm{~mA})$. The stimulus. was delivered at the subjects' right ankle, which was cleaned with alcohol, via two 8-mm Ag-AgCl Beckman electrodes (distance, $2.5 \mathrm{~cm}$ ) filled with Hewlett-Packard Redux Creme. The stimulation produces a painful, stinging sensation, which is unlike shocks caused by electric fencing, electric mains, etc

Heart rate was measured by three $8 \mathrm{~mm}$ Ag-AgCl SensorMedics electrodes. filled with Redux Creme and connected to the registration apparatus (Picker 
Schwarzer ED 14). After the skin was cleaned with alcohol, the electrodes were placed just above the sternum, at the left side of the chest, and at the left shoulder as a reference. Interbeat intervals were determined, and transformed to heart rate in beats per minute on a second-to-second basis. Heart rate response was defined as the mean change in heart rate (in beats per minute) during the $20 \mathrm{~s}$ after the onset of the stimulus, using the mean heart rate during the $10 \mathrm{~s}$ prior to onset as reference.

\section{Pharmacological manipulation and design}

A $2 \times 2$ between-subject design was employed with naltrexone (yesino) and alprazolam (Xanax) (yes/no) as factors, resulting in four cells: 1) placebo 2) naltrexone 3) alprazolam and 4) the combination of naltrexone and alprazolam. Doses were $50 \mathrm{mg}$ for naltrexone and $1 \mathrm{mg}$ for alprazolam, supplied by the University Hospital pharmacologist in capsules that had to be swallowed Subjects were randomly assigned to pharmacological condition, blind to both subject and experimenter, 11 receiving placebo, 11 receiving alprazolam, 14 receiving naltrexone and 12 receiving alprazolam and naltrexone. More subjects were assigned to the naltrexone conditions, since a dropout due to nausea was lakem into account. However, although 3 subjects reported slight nausea $(2$ receiving naltrexone and 1 receiving alprazolam and naltrexone), there was no dropout. The peak plasma concentration of naltrexone occurs within one hour. while its major metabolite 6- $\beta$-naltrexol, also an opioid antagonist, reaches peak concentration two hours after administration (AHFS, 1989). A single dose may have antagonistic effects for as long as 24 hours. Alprazolam has a half-life of more than 6 hours. Two hours after swallowing the capsule, subjects in each pharmacological condition received two stimulations, one at a current level of 2.5 $\mathrm{mA}$ and one at $5 \mathrm{~mA}$. An analysis of variance was planned with alprazolam (yes/no) and naltrexone (yes/no) as between-subject variables, and stimulation level as within-subject variable. Dependent variable was subjective pain at each level, while anxiety and positive excitement during pain stimulation were used as a manipulation check.

\section{Procedure}

Atter signing the informed consent form, the subject swallawed the capsule for pharmacological manipulation. Several questionnaires were completed and a pretest BAT was done. After resting a while, the subject performed a pen and
papar task.

Two hours after swallowing the capsule, subjects were seated in the laboratory and electrodes for pain stimulation were fastened. As part of another experment, which will be published elsewhere, the effect of pharmacological manipulation on appraach behaviour was investigated. A BAT was done four times, involving a medium-sized spider in a closed plexiglass container placed on a plateau at the end of a 2 -meter-long shelf in front of the subject. The subject was asked to perform several steps when possible, such as pulling the plateau towards her, opening the container, touching the spider with a pencil touching it with a finger, and taking the spider on the hands. The BAT was stopped when the subject indicated maximum approach by raising her hand or when four 
minutes had passed. Each of the four BATs was followed by a short confrontation with a large spider. A glass jar with the spider was brought into the room covered by a cloth, and was held in front of the subject at $50 \mathrm{~cm}$ distance. The subject was informed that the cloth would be removed and that she was supposed to watch the spider. Five seconds after the cloth was removed the spider was covered again and put at a distance. Immediately after the last confrontation, the subjecl was asked to concentrate on the forthcoming stimulation at the ankle. Stimulation of $2.5 \mathrm{~m} / \mathrm{A}$ was delivered, after which the subject rated pain anxiety and positive excitement on VASs. Next, $5 \mathrm{~mA}$ stimulation was delivered, and VASs were scored again.

\section{Results}

\section{Manipulation check}

Spider Phobia as measured by the SPQ did not differ significantly between groups receiving naltrexone vs. no naltrexome $(F(1,44)=0.48$, n.s.), or alprazolam vs. no alprazolam $(F(1,44)=0.12, n$.s.). Anxiety WAS ratings averaged over the four confrontation trials (Table 1) were not influenced by alprazolam $(F(1,44)=$ 0.07 , n.s.), whereas anxiety VAS ratings during pain stimulation were significantly lower in the alprazolam than in the no alprazolam condition (Table $2 ; F(1,44)=$ $5.22, p<0.05)$. Further, alprazolam slightly increased positive excitement during pain stimulation $(F(1,44)=3.14, P=0.08)$. Naltrexone did not significantly influence mood ratings during stimulation, neither anxiety $(F(1,44)=0.38, n . s$.) nor positive excitement $(F(1,44)=0.0, n$.s.). Finally, stimulation al $5 \mathrm{~mA}$ levelled to higher anxiety VAS ratings than stimulation at $2.5 \mathrm{~mA}$ level $(F(1,44)=12.34$, $\mathrm{p}<0.005$ ).

Table 1. Mean (SD) of the score on the Spider Phobia Questionnaire and of anxiety.

\begin{tabular}{lllll}
\hline & $\begin{array}{l}\text { Placebo } \\
n=11\end{array}$ & $\begin{array}{l}\text { Alprazolam } \\
n=11\end{array}$ & $\begin{array}{l}\text { Naltrexone } \\
n=14\end{array}$ & $\begin{array}{l}\text { Alpraz + Nal } \\
n=12\end{array}$ \\
\hline SPQ & $23.7(2.7)$ & $23.0(3.4)$ & $22.9(2.2)$ & $23.1(3.2)$ \\
VAS Con 1 & $69.9(26.9)$ & $50.7(32.0)$ & $56.9(29.3)$ & $70.0(21.2)$ \\
VAS Con 2 & $55.5(26.9)$ & $48.7(31.8)$ & $46.8(25.5)$ & $54.0(35.7)$ \\
VAS Con 3 & $49.5(22.1)$ & $44.1(29.1)$ & $43.2(28.1)$ & $48.9(32.6)$ \\
VAS Con 4 & $48.2(23.9)$ & $33.8(26.1)$ & $37.3(29.0)$ & $36.4(27.7)$ \\
\hline
\end{tabular}




\section{Subjective pain ratings}

Pain ratings were reduced by alprazolam versus no alprazolam, but the effect was only marginally significant (see Figure $1 ; F(1,44)=2.94, p=0.09$ ). Pain ratings were not influenced by naltrexone $(F(1,44)=0.29$. n.s.). nor was there any interaction between alprazolam and naltrexone $(F(1,44)=0.39$, n.s.). Stimulation at $5 \mathrm{~mA}$ level led to significantly higher pain ratings than stimulation at $2.5 \mathrm{~mA}$ level $(F(1,44)=48.14 ; p<0.001)$, but there were no differential effects of the experimental conditions at the two stimulation levels.

\section{Heart rate}

Due to disturbance of measurement during electrical stimulation, heart rate data of five subjects were lost (one placebo, two alprazolam and two naltrexone and alprazolam). Mean heart rate was higher during the $10 \mathrm{~s}$ before the first $(2.5 \mathrm{~mA})$ than before the second stimulation $(5 \mathrm{~mA})$, but was unaffected by alprazolam or naltrexone. Surprisingly, mean heart rate during $20 \mathrm{~s}$ after the start of stimulation decreased with respect to the $10 \mathrm{~s}$ prior to stimulation $(F(1,39)=8.55 ; p<0.01)$. This deceleration was observed at $2.5 \mathrm{~mA}$, but not at $5 \mathrm{~mA}$ stimulation level $(-4.5$ vs. $-0.4 \mathrm{bpm}, F(1,39)=14.96 ; p<0.001$ ). Heart rate response did not show effects of naltrexone or alprazolam, nor an interaction effect.

Table 2. Mean (SD) of anxiety, positive excitement and pain ratings during, and heart rate before and heart rate response after $2.5 \mathrm{~mA}$ and $5 \mathrm{~mA}$ stimulation, displayed for each pharmacological condition.

\begin{tabular}{llllll}
\hline & mA & $\begin{array}{l}\text { Placebo } \\
n=11\end{array}$ & $\begin{array}{l}\text { Alprazolam } \\
n=11\end{array}$ & $\begin{array}{l}\text { Naltrexone } \\
n=14\end{array}$ & $\begin{array}{l}\text { Alpraz }+ \text { Nal } \\
n=12\end{array}$ \\
\hline Anxiety VAS & 2.5 & $21.1(24.6)$ & $15.3(17.5)$ & $23.4(22.5)$ & $8.0(6.2)$ \\
& 5 & $32.4(26.5)$ & $22.5(27.1)$ & $34.1(27.3)$ & $11.0(14.0)$ \\
\hline Positive VAS & 2.5 & $16.5(18.9)$ & $49.8(35.4)$ & $32.3(30.7)$ & $33.3(35.7)$ \\
& 5 & $21.2(26.2)$ & $45.5(36.9)$ & $30.8(30.2)$ & $36.3(37.4)$ \\
\hline Pain VAS & 2.5 & $30.8(32.8)$ & $26.3(31.9)$ & $30.0(29.3)$ & $13.8(22.7)$ \\
& 5 & $61.2(35.2)$ & $46.8(35.8)$ & $52.8(37.5)$ & $39.2(32.0)$ \\
\hline HR pre & 2.5 & $74.4(10.0)$ & $82.3(11.7)$ & $79.7(16.1)$ & $75.0(8.8)$ \\
& 5 & $70.4(11.7)$ & $77.9(8.4)$ & $79.1(15.7)$ & $72.5(7.3)$ \\
\hline HR response & 2.5 & $-5.2(5.1)$ & $-4.3(12.3)$ & $-4.3(4.8)$ & $-4.4(4.8)$ \\
& 5 & $-1.1(5.9)$ & $1.9(5.9)$ & $-1.1(6.8)$ & $-0.9(3.2)$ \\
\hline
\end{tabular}



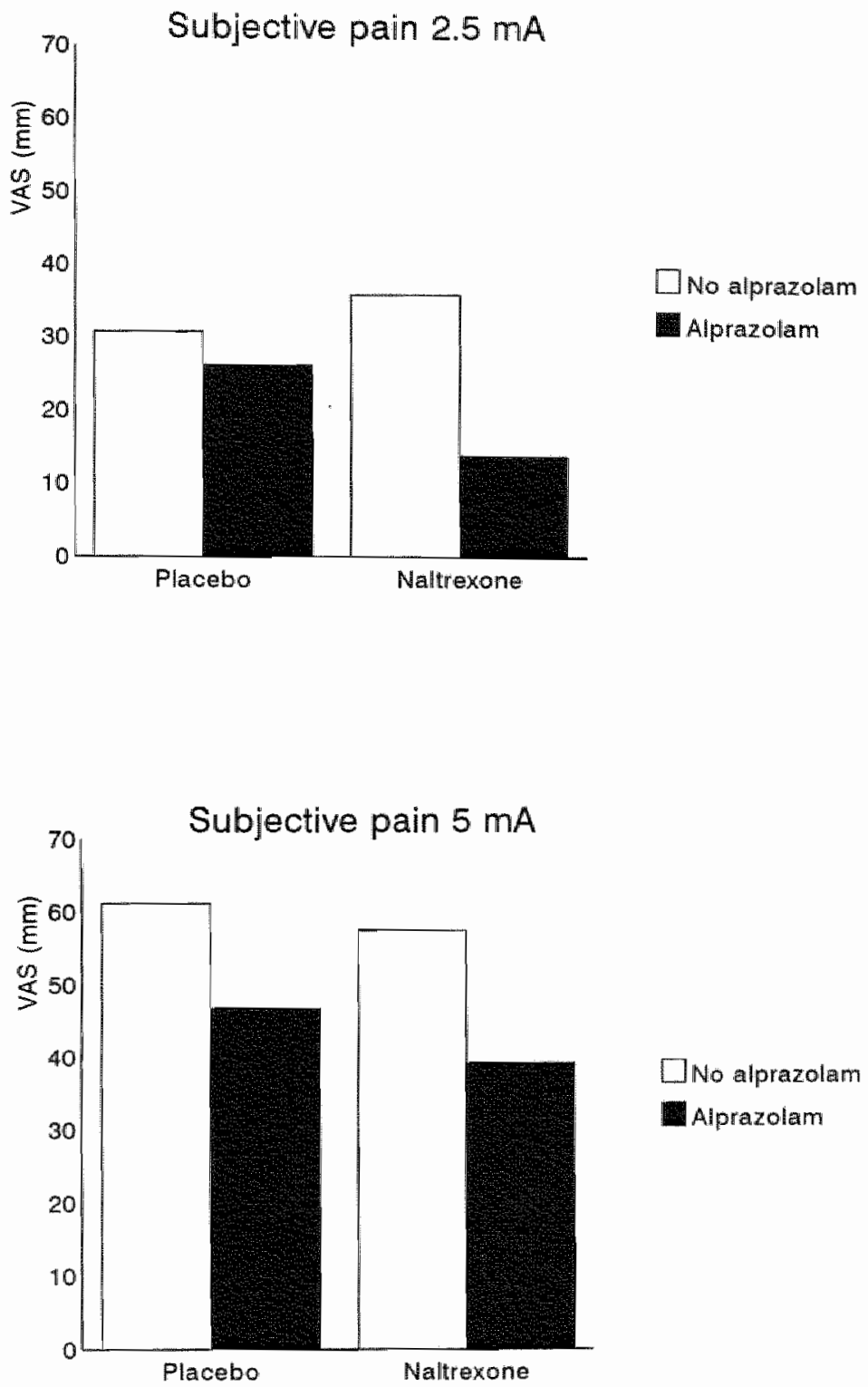

Figure 1. Mean subjective pain ratings for low $(2.5 \mathrm{~mA})$ and high $(5 \mathrm{~mA})$ stimulation level, displayed for each pharmacological condition. 


\section{Discussion}

Neither reducing anxiety by alprazolam nor blocking endogenous opioids by naltrexone led to a pain increase compared to placebo. Despite the high level of anxiety induced by confrontation with the spider, no endogenous opioid-mediated effects were found. Naltrexone did not affect subjective pain due to electrical stimulation, nor did if influence mood during a phobic fear situation. This means that hypothesis 1, stating that phobic fear is associated with a pain reduction mediated by the release of endogenous opiaids, must be refuted. Alprazolam, however, which compared to placebo was associated with a clear decrease in anxiety and a slight increase in positive mood during pain stimulation, showed a marginally significant pain-inhibiting effect. This is consistent with hypothesis lla, stating that anxiety increases pain through enhanced arousal or sympathetic outflow. Heart rate responses to pain did not show such an inhibition by alprazolam. It is surprising that irrespective of condition, heart rate showed a deceleration in response lo low current painful stimulation, since the defensive reaction to pair is believed to involve a heart rate acceleration (Turpin, 1986), Perhaps pain at low current level was not strong enough to induce a defensive reaction. Also, the announcement of the pain stimulus immediately prior to the start of stimulation may already have resulted in a heart rate acceleration, masking responses to the pain.

Although the mean decrease in pain in the alprazolam witth respect to the no alprazalam condition was pronounced, statistically it was only marginally significant. Therefore, it cannot be excluded that the effect relied on chance. Part of the problem may be the lack of a baseline pain rating: since the subjective intensity of the pain stimulus differed substantially between individuals, the error variance may have become too large. Assuming, however, that the reduction in subjective pain by alprazolam reflects real processes, what mechanisms could have beem responsible? Since there was no interaction with naltrexone, it is not likely that in the present experiment the pain-inhibiting effects of alprazolam were mediated by endogenous opioids (hypothesis llb), as suggested by Tripp \& McNaughton (1992). Neither can these findings be explained by assuming opioid antagonist effects of benzodiazepines (Rovati et al, 1990; Gear et al., 1997), for in that case pain=increasing effects of alprazolam compared to placebo would be expected, next to similar effects of naltrexone. Therefore, in line with Dellemijn \& Fields (1994), who argue that benzodiazepines may have analgesic effects by reducing situational anxiety, the reduction in pain is likely to be related to the observed reduction in anxiely during pain stimulation. The fact that alprazolam did not affect anxiety ratings during confrontations with the spider, suggests that only anxiely associated with the pain was reduced. Interestingly, some researchers have proposed that pain-related anxiety may increase the experience of pain since arousal associated with anxiety is attributed to the pain, whereas pain-irrelevant anxiety would not increase pain (Nisbett \& Schachter, 1966; Weisemberg et al., 1984; Dougher et al. 1987; Al-Absi \& Rokke, 1991). Thus, arousal induced by anticipation of painful stimulation may have been labeled as pain, increasing the pain experience, with alprazolam resulting in less arousal to be attributed to the pain and thereby reducing the pain experience. A second 
possibility is that effects of alprazolam were not mediated by arousal, but by differences in attentional focus. Several studies have indicated that anxiety, either pain-relevant or pain-irrelevant, does not influence subjective pain when focus of attention is controlled for, while attention on pain leads to higher pain ratings than attention distracted from pain (Arntz, Dreessen \& Merckelbach, 1991; Arntz \& de Jong 1993; Arntz, Dreessen \& de Jong, 1994; Janssen \& Arntz, 1996). Perhaps the reduction in pain-related anxiety in the alprazolam group, despite instructions to concentrate on the pain, resulted in less altention towards the painful stimulus. in turn leading to a reduction in subjective pain. Finally, it is possible that alprazolam, by attenuating anxiety during painful stimulation, inhibited sympathetic activation and catecholamine release. There is evidence that catecholamines peripherally sensitize nociceptors, resulting in hyperalgesia (Chapman \& Turner, 1986; Drummond, 1995). However, these effects may only be of importance in injured or sensitized skin (Janssen, Arntz \& Bouts, 1998).

Despite strong levels of anxiety, no endogenous opioid-mediated analgesia was induced in a phobic situation, compatible with previous negative findings (Janssen \& Arntz, 1997). Although phobias have led to some of the strongest subjective and psychophysiological reactions in human subjects under laboratory conditions, they may be accompanied by weak or even absent endocrine responses (see Curtis \& Glitz, 1988 for a discussion of neuroendocrine findings in anxiety disarders). One of the reasons may be that learned helplessness or uncontrollability is a necessary condition for stress induced analgesia, as was demonstrated in animal studies (Maier et al., 1983; Maier, 1990). However, even under the relatively uncontrollable situation of a "flooding"-like confrontation with a spider, no effects of an oploid antagonist were faund. A more uncontrollable situation is "mainly for ethical reasons, hard to achieve in the human laboratory. Henry (1990) claimed that different kinds of stress may lead to differentendocrine responses, catecholamine release prevailing when fight or flight behaviour is required, whereas the adrenal cortical axis is activated when the subject is helpless and not in control of the situation. In a study with subjects exposing themselves to the more real-life and uncontrollable stress situation of a first-time parachute jump, an opioid-mediated analgesia was found (Janssen \& Arntz. submitted), supporting the idea that endogenous opioids are released in siluations in which threat is both high and uncontrollable. 



\title{
Uncontrollable stress and opioid-mediated analgesia in novice parachute jumpers
}

\begin{abstract}
Summary
This study investigated whether, in an owerwhelmingly stressfull and uncontrollable situation, an opioidmediated analgesia could be demonstrated in human subjects. In subjects performing a first-time parachute jump, sensitivity for an electrical and a pressure pain stirnulus was measured at several moments before and after the jump. The opioid antagonist naloxione or saline was administered immediately after the jump to examine effects of endogenous opioids on the pain measures. Blood plasma levels of j-endorphin, epinephrine and norepinephrine were determined at pretest, before and after the jump. After the jump, a decrease in pain was shown in the placebo group with respect to the naloxone group, which is consistent with an opioid-induced analgesia. Also, plasma $\beta$-endorphin had tripled after the jump, associated with anxiety and loss of control during the jump. However, the placebo group did not show an analgesia compared with measures before the jump. The data suggest that at the same time another, nonopioid process was activated, which increased pain while endogenous opioids attenuated it.
\end{abstract}

\section{Introduction}

The phenomenon of stress-induced analgesia has been demonstrated in many animal studies (Maier et al., 1983). Since stress-induced analgesic effects can be reversed by an opioid antagonist, they are usually attributed to the release of endogenous opioids. However, only a few studies (Pitman et al., 1990; Willer \& Ernst, 1986) support the existence of a similar stress-induced analgesic mechanism in humans. In clinical practice, stress or sympathetic activation is even believed to exacerbate pain experience (Dougher, Goldstein \& Leight, 1987), anxiety reduction techniques being used to minimize the pain (Chapman \& Bonica, 1983; Chapman \& Turner, 1986). There is also evidence that, although endocrine responses significantly influence pain perception in animals, cognitive factors like attention (Arntz, Dreessen \& Merckelbach, 1991; Arntz, Dreessen \& de Jong, 1994; Arntz \& de Jong, 1993) are more important in humans.

Two previous studies examined the possible role of endogenous opioid activity by administering an opioid antagonist to spider phobics who were confronted with a spider (Janssen \& Arntz, 1996; 1997). Despite high levels of anxiety, no selective effect of the opioid antagonist was found on pain responses, nor did anxiety increase $\beta$-endorphin plasma levels. Although it has been demonstrated that animal phobias can induce significant endocrine responses (Nesse et al., 1985), they can also be accompanied by weak or even absent endocrine 
responses (Curtis \& Gitz, 1988). Human laboratory studies may lack some of the characteristics of animal studies, most notably the low degree of control the animal has over the experimental situation. Indeed, there are indications from studies with rats that uncontrollability is a necessary condition for stress-induced analgesia (Maier et al, 1983). Also, Henry (1990) claimed that different kinds of stress may lead to different endocrine responses, catecholamine release prevalling when fight or flight behaviour is required, while activation of the adrenal cortical axis is restricted to situations in which the subject is more or less nelpless. The relatively controllable situation in the laboratory may have accounted for the absence of endorphinergic activity in the spider phobics. Therefore, research on stress-induced analgesia in humans should involve a situation which mimics uncontrollable real danger in an ethically acceptable way. Several investigators have used a parachute jump, which could be argued to possess these characteristics, to induce a state of real-life stress in humans under controlled experimental conditions (Fenz \& Epstein, 1967; Schedlowski et al., 1995; Ursin, Baade \& Seymour, 1978). Rises in plasma $\beta$-endorphin after parachute jumping (Schedlowski et al., 1995) and bungee jumping (Hennig, Laschefski, Opper, 1994) were found to correlate respectively with locus of control measures and feelings of euphoria. Autonomic arousal measures in novice parachutists have been shown to gradually increase until the moment of the jump (Fenz \& Epstein, 1967; Fenz \& Jones, 1972). For the present study a first-time parachute jump was chosen, during which subjects were physically attached to an instructor. The question was addressed whether, in an overwhelmingly stressful situation outside the laboratory, stress-induced analgesia could be demonstrated in human subjects. The sensitivity for an electrical and a pressure pain stimulus was measured at different times before and after the jump, as well as subjective anxiety, perceived control and euphoria. To examine effects of endagenous opioids released during the jump, either the opioid antagonist naloxone or placebo was administered immediately after the jump. $\beta$ endorphin plasma levels, a rise in which may be considered a biologic marker of the human stress response and may be associated with analgesic effects (Cohen, Pickar \& Dubois, 1983), were determined as a manipulation check. Also, epinephrine and norepinephrine plasma levels were determined, because catecholamine release may be stimulated by naloxone during extreme sympathetic activation (Bouloux et all., 1985). There is evidence that peripheral norepinephrine sensitizes nociceptors after nerve injury, but also, to a llesser degree, in normal skin (Drummond, 1995; 1996). In a study in which epinephrine was infused systemically in natural doses, we found a hyperalgesia in both sensitized and normal skin (Janssen, Arntz \& Bouts, 1998). Therefore, plasma levels are needed to control for possible effects of naloxone on pain mediated by catecholamines. During the jump an increase in anxiety and a decrease in perceived control ratings was expected, associated with plasma $\beta$-endorphin release, while euphoria was expecied to be increased after the jump. The hypothesis was tested whether, due to endogenous opioids, subjects receiving placebo would show a pain inhibition after the jump with respect to subjects
receiving naloxane. 


\section{Method}

\section{Subjects}

Twenty-four subjects, 12 male and 12 female, were recruited through advertisements in the newspaper, offering a $30 \%$ discount off the price of a firsttime parachutejump in return for participation in the experiment. None of the subjects had ever jumped before. Two groups of subjects were formed, matching in age (19-40, mean age 28, SD 6) and sex. Subjects scored slightly below population mean on STAI trait anxiety (33.7. SD 7.1, fourth decile), and above mean on the Sensation Seeking Scale (13.0, SD 2.1, seventh decile). Exclusion criteria were cardiovascular disease, liver or kidney dysfunction, use of opiate medication and alcohol or drug abuse. Subjects signed an informed consent form after being informed about the procedure, which was approved by our Medical Ethics Committee.

\section{Materials}

Subjects completed the Sensation Seeking Scale (Fey \& van Zuilen, 1984; Zuckerman, 1979), a shortened version of Rotter's InternaluExternal Control Scale (Andriessen \& van Cadsand, 1983; Rotter, 1966) and the Trait Anxiety Index (STAI, Spielberger et al., 1970). Anxiety, euphoria and perceived control were rated on 100-mm visual analogue scales (VASs) ranging from "not at all ..." to "extremely ...", as were subjective pain produced by an electrical stimulus and amount of attention paid to the stimulus. An electrical pain stimulus of $6 \mathrm{~mA}$ (10ms rectangular pulses, with an interpulse time of $10 \mathrm{~ms}$ ) was generated by a Phy-action 300 (Uniphy). Via two disposable electrodes (Medicotest) two stimuli of each $2 \mathrm{~s}$ (10 s apart) were applied to the ankle, which was rubbed with SkinPure (Nihon Kohden) to reduce skin resistance. Pressure pain was produced by a Forgione-Barber stimulator (Forgione \& Barber, 1971), applying a $1.7 \times 16$ $\mathrm{mm}$ plexiglass wedge with a pressure of $62.5 \mathrm{gram} / \mathrm{mm}^{2}$ to the middle phalanx of the second finger of the nondominant hand. This usually begins with a sensation of weight, becoming painful after some time and, finally, intolerable. Maximum time was $4 \mathrm{~min}$. Pain threshold and tolerance were measured with a stopwatch.

\section{Blood samples and pharmacological manipulation}

For blood taking and pharmacological manipulation a catheter (Venflon 1.0/32 $\mathrm{mm}$ ) was inserted in the anterior cubital vein. After the jump either $10 \mathrm{~mL}$ saline placebo, or $4 \mathrm{mg}$ of naloxone hydrochloride $(0.4 \mathrm{mg} / 1 \mathrm{~mL})$ was administered intravenously. At pretest instruction, and landing time, $5 \mathrm{~mL}$ blood was collected in tubes on ice containing glutathione and heparin for the catecholamine analysis, and $5 \mathrm{~mL}$ in heparim-containing tubes on ice for the analysis of plasma $\beta$ endorphin. All samples were spun down at $3000 \mathrm{rpm}$ for $10 \mathrm{~min}$ at $0^{\circ} \mathrm{C}$ and stored at $-40^{\circ} \mathrm{C}$. Plasma catecholamines were analyzed by High-Performance Liquid chromatography and fluorescent detection (van den Hoorn et al., 1989). $\beta$-endorphins were assayed by a solid phase two-site immunoradiometric assay (IRMA, Nichols Institute Diagnostics, The Netherlands). Sensitivity was $9 \mathrm{pg} / \mathrm{mL}$, intra-assay and inter-assay coefficients of variation were $4 \%$ and $8 \%$, 
respectively. The antibody exhibited $16 \%$ crossreactivity with human $\beta$-lipotropin, and less than $0.01 \%$ with alpha- and gamma-endorphin, the enkephalins, alpha MSH and dynorphin. Due to sampling failures, catecholamine values of three subjects and $\beta$-endorphin values of four subjects were missing. For determining the correlations between blood measures and subjective measures, only those subjects were used of which blood data were actually collected. However, for the analyses with pain measures, release was estimated in a conservative way on the basis of a regression equation with naloxone condition, pain difference score and anxiety and perceived control during the jump as predictors

\section{Design}

Naloxone or saline placebo was administered between subjects immediately after the jump, blind to both subject and experimenter. Age, sex and time of jumping were controlled for by matching on these variables. Subjective pain produced by the electrical stimulus was measured within-subject at five different times (at pretest, after instruction, in the airplane, after landing and at posttest), pain threshold and tolerance for the pressure stimulator at three different times (at pretest, after instruction and after landing). Anxiety, euphoria, perceived control and attention VASs were rated as a manipulation check. Blood was taken three times (at pretest, after instruction and after landing) to detect $\beta$-endorphin and catecholamine release induced by the jump, the first as a manipulation check, the latter to be used as covariate to control for possible effects of naloxone on (nor)epinephrine. The ankle used for electrical stimulation (left vs. right) was counterbalanced across pharmacological condition. To analyze the pain data, an analysis of variance was planned with pain measure (subjective pain vs. pain threshold vs. pain tolerance) as within-subject factor and pharmacological manipulation (naloxone vs. placebo) as between-subject factor. Dependent variables were subjective pain, pain threshold and pain tolerance after the pharmacological manipulation at landing, corrected for interindividual differences in sensitivity by subtracting the mean of prejump measurements. To obtain equal scaling, values of the three different pain measures were transformed into $z$ scores. Furthermore, a multiple regression was planned with pain measures as dependent variables and manipulation check variables as predictors.

\section{Procedure}

On the morning of the jump, subjects came to the laboratory at $0800 \mathrm{hr}$ in groups of 4-6. They were medically checked by a doctor and informed about the procedures. After the catheter was inserted and the electrodes were fastened, subjects filled in the questionnaires. Thirty minutes later the first blood sample was taken. After rating anxiety, euphoria and perceived control on the VASs, each subject received two electrical stimuli with the instruction to focus attention on them. Subjective pain and amount of attention paid to the two stimuli were then rated on VASs. Subjects were told that during the rest of the experiment the stimuli would be of the same intensity, but that they were supposed to rate their experience of the pain. Then, the pressure stimulator was put on the second finger of the nondominant hand, and the subject was asked to concentrate on the stimulus and to indicate pain threshold and the point at which the pain became 
intolerable. After the last subject, blood was spun down and participants were taken to the parachute centre.

Immediately before the jump, having received instructions in pairs of two, subjects were seated outside looking out over the runway. Here, they were asked to fill in the STAl state questionnaire and anxiety, euphoria and perceived control VASs, after which blood was taken. Then subjects rated pain and attention on VASs after receiving the two electrical stimuli, and indicated pain threshold and tolerance for the pressure stimulator. Subsequently subjects boarded the airplane, where, in the air, mood and experience of the two electrical stimuli were rated again.

Subjects performed a tandem-parachute jump (between 1330 and $1700 \mathrm{hr}$ ), meaning that during the jump they were physically attached to an instructor. They jumped from an altitude of $4.5 \mathrm{~km}$ (2.8 mile), the parachute opening at $1 \mathrm{~km}(0.6$ mile) above the ground, after approximately $60 \mathrm{sec}$ free-fall time. At the dropzone, immediately after the jump, naloxone or placebo was administered, followed by the mood VASs and a 2 min waiting period (the onset of action is 1-2 min). After $2 \mathrm{~min}$, blood was sampled, experience of electrical stimuli was rated and pressure pain threshold and tolerance were measured. Finally, subjects were taken back from the dropzone to the centre, where mood and experience of electrical stimuli were rated once more after an hour.

\section{Results}

\section{Manipulation check}

The manipulation check VASs at pretest; instruction, in the airplane and during the jump were analyzed with a MANOVA trend analysis, with condition as between-subject factor and the four tests as within-subject factor (decomposed into trends). As shown in Figure 1, anxiety ratings increased until the moment of the jump (linear trend $t(23)=2.5, p<0.05$ ), while perceived control decreased (linear trend $\mathrm{t}(23)=-2.8, p<0.05$ ), anxiety and control during the jump being neglatively correlated $(r=-0.48, p<0.01)$. Ratings of anxiety, euphoria or attention on the electrical pain stimulus did not differ significantly between groups at any moment, but perceived control during the jump was slightly lower in the placebo group $(t(22)=-1.7, p=0.10)$.

While $\beta$-endorphin plasma levels had not increased with respect to the pretest before the jump, the average had tripled immediately after jumping (see Figure $2 ; F(1,18)=17.4, p<0.001)$. This increase correlated positively with epinephrine release $(r=0.76, p<0.001)$, positively with anxiety $(r=0.42, p<0.05)$, and slightlly negatively with perceived control during the jump $(r=-0.35, p=0.07)$, but not with increased euphoria after the jump $(r=0.07)$ or norepinephrine release $(r=0.18$; Pearson correlations, one-tailed). Epinephrine showed a marginal increase with respect to the pretest $(F(1,19)=3.48, p=0.08)$, while norepinephrine had significantly increased $(F(1,19)=29.4, p<0.001)$. Naloxone did not significantly influence $\beta$-endorphin release $(F(1,18)=0.4$ ), epinephrine release $(F(1,19)=1.0)$ or norepinephrine release $(F(1,19)=1.2)$. 

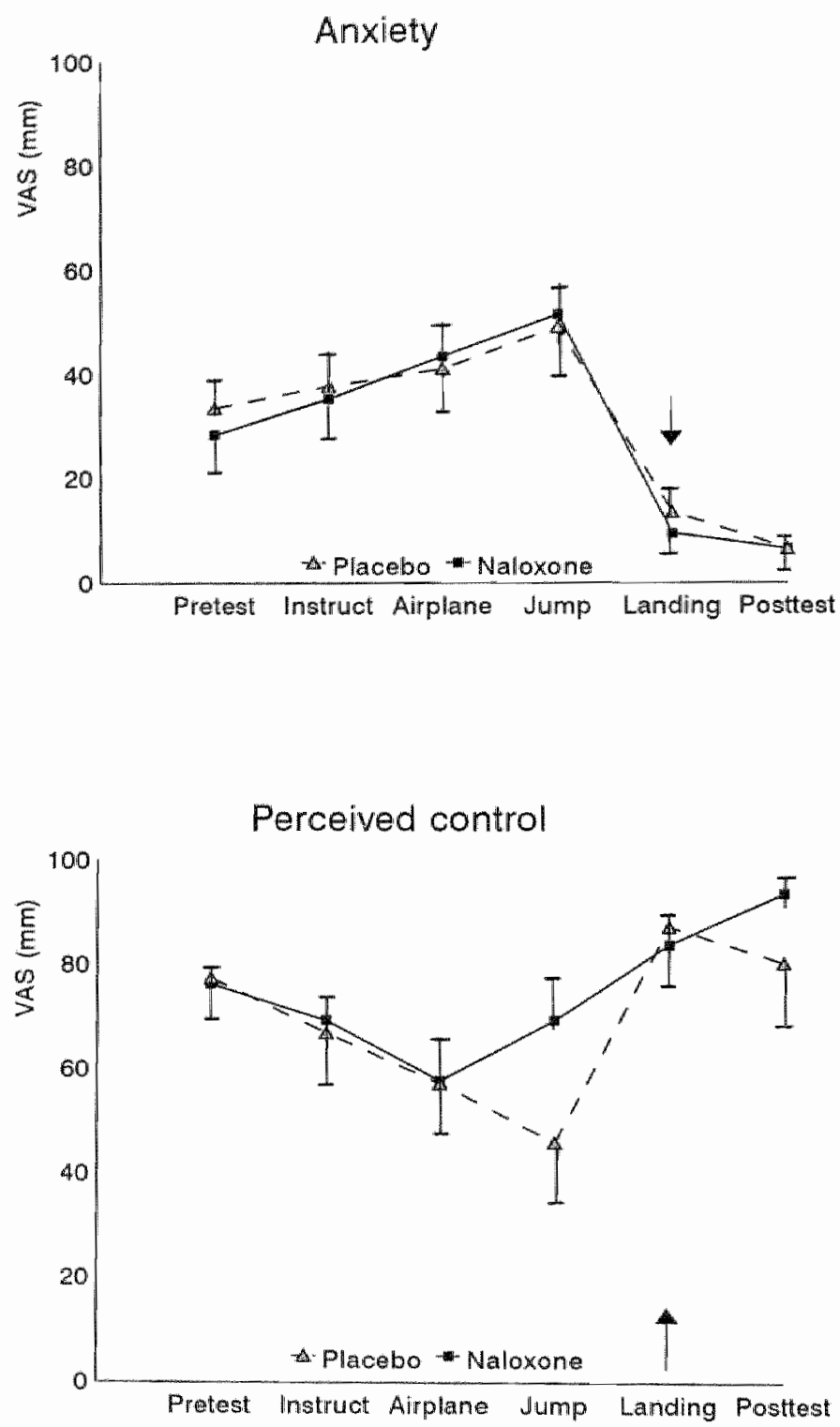

Figure 1. Arriety and perceived control ratings at several points in time before and after the jump, displayed for naloxone and placebo group (administration indicated by arrow). 

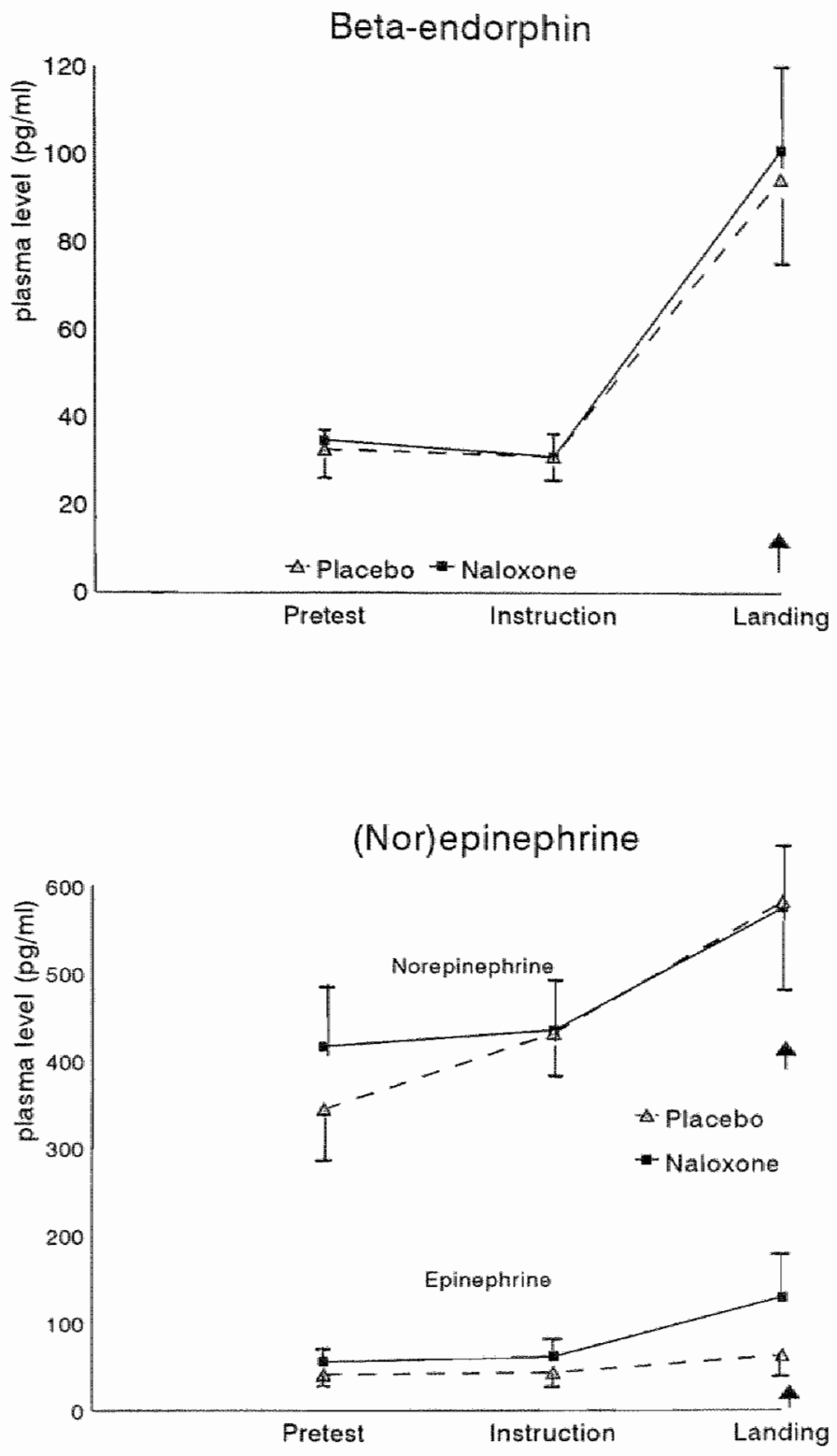

Figure 2. Endocrine levels at pretest, instruction and landing, displayed for naloxone and placebo group (administration indicated by arrow). 


\section{Pain measures}

To meet the normal distribution assumption for analysis of variance. pressure pain thresholds were logarithmically transformed. Since half of the subjects showed no change from before to after the jump as the result of a celling effect, pressure pain tolerance was not analyzed. Electrical pain and pressure pain threshold after the jump were corrected for individual differences by subtracting the mean of measurements before the jump, before administration of naloxone or placebo. A $2 \times 2$ analy sis of variance was done, with $z$-scores of electrical pain and (the negative of threshold as dependent variables, pain measure as withinsubject factor and maloxone group as between-subject factor. This analysis showed that naloxone increased pain compared to placebo $(F(1,22)=5.6 . p<$ $0.05)$, without interaction between naloxone and pain measure $(F(1,22)=0.02$; n.s.). To control for differences between naloxone and placebo in perceived control or (nor)epinephrine levels, another analysis of variance was done with these variables as covariates. Again, naloxone was shown to increase pain compared to placebo $(F(1,19)=8.2, p<0.05)$ *

To investigate which variables contributed to pain sensitivity after the jump, a regression analysis was done with pain as dependent variable. Since the interaction between naloxone and pain measure was not significant, the $z$-scores of electrical pain and pressure pain threshold were added to produce a single measure for pain sensitivity. A backward regression analysis was performed with as predictors anxiety and perceived control during the jump, euphoria after the jump, I-E control scalle, epinephrine and norepinephrine release, and $\beta$-endorphin release, which (given the blocking properties of naloxone) was assumed zero for the naloxone group. Pain sensitivity after the jump was shown to be negatively related to $\beta$-endorphin release $(T=-3.4, p<0.005)$. Furthermore, perceived control during the jump was negatively related to pain $(T=-2.3, p<0.05)$, while epinephrine release was slightly positively related to pain $(T=1.9, p=0.08)$. *

\footnotetext{
* These analyses were done with estimated values for the missing blood samples. However, the resuls did not differ from the analyses including only the data that were actually collected.
} 

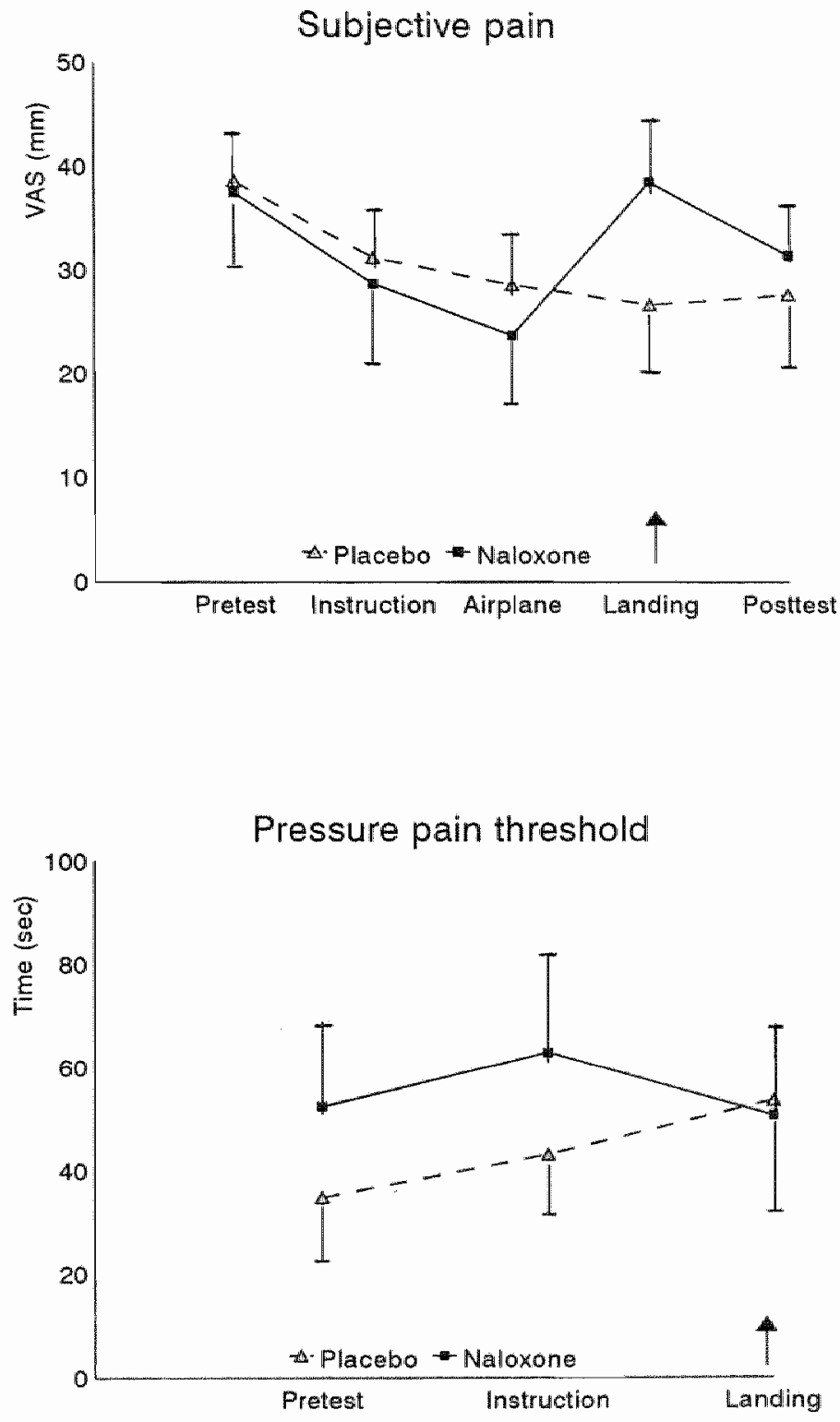

Figure 3. Subjective pain as a result of the electrical stimulus and pressure pain thresholld at several points in time before and after the jump, displayed for nalloxone and placebo group (administration indicated by arrow). 


\section{Discussion}

In congruence with many animal studies demonstrating stress-induced analgesia, this study provides evidence that an opioid-mediated analgesia can be induced in human subjects as a result of a real-life stressful situation. After a first-time parachute jump, subjects receiving placebo reported lower pain sensitivity than subjects receiving the opioid antagonist naloxone, which is in line with our hypothesis. The effect was accompanied by a profound increase in plasma $\beta$ endorphin after the jump, which suggests that opioid processes were triggered by the jump. Also, $\beta$-endorphin release seemed related to feelings of anxiety and loss of control during the jump.

Can the lower pain sensitivity in the placebo condition compared to the naloxone condition exclusively be interpreted as an opioid-mediated analgesia, or do alternative explanations exist? Firstly, naloxone is known to stimulate catecholamine release during stress, possibly by reversing catecholamineinhibiting effects of endorphins (Bouloux et al., 1985). Consistent with reports that catecholamines may sensitize nociceptors (Chapman \& Turner, 1986, Drummond, 1995 1996; Janssen, Arntz \& Bouts, 1998), naloxone may have enhanced catecholamine release and consequently increased pain. However, naloxone did not significantly influence catecholamine release, and the effect of naloxone on pain did not disappear when corrected for epinephrine and norepinephrine plasma levels, reducing the likeliness of this interpretation. Secondly, the placebo group showed lower perceived control during the jump than the naloxone group. However, this difference could not have mediated the effect of naloxone on pain, since perceived control as a covariate did not disturb the effect. It is important to note that the difference in perceived control cannot be attributed to pharmacological manipulation, since naloxone was administered after the jump, immediately before rating, and has an onset of action of 1-2 min. Thirdly, the effect of naloxone versus placebo cannot be attributed to a rating bias, since pharmacological manipulation was blind to both subject and experimenter. Finally, it is unlikely that naloxone had a direct increasing effect on pain, since naloxone seems to have agonistic, pain-inhibiting effects when there is no opioid activity to be antagonized (Janssen \& Arntz, 1997; Stacher et al., 1988). Therefore, the conclusion is warranted that the naloxone effect is likely to reflect an opioid analgesia.

Although the effect of naloxone versus placebo was in line with expectation, it is not clear what exactly triggered this opioid-mediated analgesia. From Figure 3 it appears that, rather than showing a sudden decrease after the jump compared to measurements just before the jump, pain sensitivity in the placebo group showed a small reduction, while pain returned to pretest level when naloxone was administered. These data can be interpreted in at least three ways: a) An opioid-mediated, naloxone-sensitive habituation may have preceded the jump. This may explain the reduction in pain sensitivity with repeated testing, and the return to pretest level when naloxone was administered. However, $\beta-$ endorphin plasma levels did not increase before the jump.

b) Anticipatory anxiety may have caused an opioid-mediated analgesia before the jump. Although plasma $\beta$-endorphin levels were not raised before the jump, an 
analgesia due to central opioid activity prior to the jump cannot be ruled out. since opioid analgesic effects have been found without rises in $\beta$-endorphin plasma levels (Pitman et all. 1990).

c) The huge increase in $\beta$-endorphin plasma levels immediately after the jump. however, strongly suggests that opioid mechanisms were triggered at the moment of the jump and not before. Assuming that opioid mechanisms caused the abserved analgesia in the placebo group with respect to the naloxone group: subjective pain and pain threshold in both groups seem shifted upward and downward respectively. Thus, superimposed on the naloxone-reversible analgesia, a second, nonopioid process may have been triggered by the jump. increasing pain in both groups while endogenous opioids were attenuating it in the placebo group. Although pain in the naloxone group did not exceed pretest levels, this could be attributed to a habituation over time. The speculative, but interesting assumption of a second process is supported by the regression analy sis, in which perceived control during the jump showed a significant negative relationship to pain (less control was associated with increased pain) when the effect of $\beta$-endorphin release was partialled out. This suggests that, while loss of control led to the release of endogenous opioids, it may at the same time have effected a pain increase. This is consistent with studies showing that lack of control increases pain sensitivity with respect to perceived control (Arntz \& Schmidt, 1989). Although most studies reporting a beneficial effect of control examined the effect of pain-related control, lack of perceived control during the jump may have generalized to other areas and increased later pain. Thus, while loss of perceived control during the jump may have triggered the release of endogenous opioids, resulting in a naloxone-reversible analgesia, simultaneously, through other pathways (perhaps adrenergic?). loss of control may have effected a pain increase in both groups.

Although the patterns of anxiety and control ratings and their correlations with plasma $\beta$-endorphin suggest that the observed effects were a result of psychological stress, the absolute values of anxiety and control were surprisingly little affected by the jump. It could be argued that in these parachute jumpers, being less trait anxious and scoring higher on the sensation seeking scale than the normal population, extreme shifts cannot be expected. Subjects may have preferred to label their anxiety as excitement, as suggested by Roth ot al (1996), or even as euphoria. On the other hand, the question may be raised whether parachute jumping is an ideal model of psychological stress, since it includes several physical stress components (e.g. changes in temperature and hypobaric pressure) that cannot be easily disentangled from psychological factors (HoffmanGoetz \& Pederson, 1995). The fact that most animal studies demonstrating stress-induced analgesia also used physical stressors, such as cold swims, hot plates or electrical stimulation, could suggest that endogenous opioid activity is determined mainly by physical factors. However, since analgesic effects in animal studies are restricted to situations in which threat is uncontrollable, they have been attributed to fear (Fanselow, 1991). Interestingly, the few studies demonstrating a stress-induced analgesia in humans also used stressors related to physical threat, such as highly noxious elecirical stimulation (Willer \& Ernst, 1986) or a combat-video (Pitman et al., 1990). Likewise, the experience of falling 
during a parachute jump may have triggered a sense of physical threat. Perhaps endogenous opioids help to diminish the impact of actual or imminent physical harm when all other ways of coping have failed. 


\section{Anxiety and pain: epinephrine- induced hyperalgesia and attentional influences}

\section{Summary}

The role of peripheral epinephrine in the influence of anxiety on pain was investigated by intravenously infusing epinephrine and placebo in three increasing doses. The effect of epinephrine was measured within shlojects on several subjective and autonomic measures: subjective pain, skin conductance response and heart rate response due to electrical stimulation, threshold for heat pain, and threshold for pressure pain. Heat pain threshold was measured both on normal skin and on skin sensitized with capsaicin, since there is evidence that effects of sympathetic stimulation are only to be found in damaged or sensitized skin. Epinephrine caused a slight increase in subjective pain due to electrical stimulation, and a decrease in heat pain threshold, which was larger on capsaicin-treated than on normal skin. However, heart rate response due to electrical stimulation and pressure pain threshold were not significantly influenced, while skin conductance response was aven inhibited by epinephrine. Attentional focus, which was manipulated within electrical stimullation, seemed to have a much stronger influence on pain responses than pharmacological manipulation, independent of epinephrine. It may be speculated that, although evidence was found for effects of epinephrine on pain, they may be overruled by effects of attention.

\section{Introduction}

In both clinical and experimental settings, anxiety and the experience of pain are sometimes found to be positively related. It has been hypothesized that anxiety increases pain through the release of catecholamines, peripherally sensitizing or even stimulating nociceptors (Chapman \& Turner 1986 ). This idea is supported by research on Sympathetically Maintained Pain (SMP), a chronic pain state that can be alleviated by sympathetic block or sympathectomy (Wiesenfeld-Hallin \& Hallin, 1984). In damaged or sensitized skin, stimulation of the sympathelic nerve or injection of (nor)epinephrine has been shown to activate nociceptors in animals (Hu \& Zhu, 1989; Sato \& Perl, 1991) or to increase thermal hyperalgesla and mechanical allodynia in humans (Drummond, 1995; Choi \& Rowbotham, 1997), while administration of adrenergic antagonists attenuated mechanical allodynia (Liu et al., 1996; Kinnman, Nygårds \& Hansson, 1997). Although effects on pain are not always found in normal skin (Roberts \& Elardo, 1985; Shea \& Perl, 1985; Barasi \& Lynn, 1986), these findings suggest that sympathetic activation influences pain and may increase it during anxiety. This is in contrast with Bolles and Fanselow (1980), who postulated the view that anxiety inhibits pain through 
the release of endogenous opibids. Their view is supported by animal studies (e.g. Maier et al., 1983) and some studies with humans (Willer \& Emst, 1986; Pitman et al., 1990; Janssen \& Arntz, submitted). Clearly, research on the relationship beween anxlety and pain so far has not led to unequivocal conclusions, and underlying mechanisms are not fully understood.

The central aim of the present study was to investigate whether epinephrine, which is one of the main endocrine substances released during anxiety, induces a hyperalgesia when infused systemically. Systemical infusion of epinephrine leads to enhanced concentrations in skin tissue, as during sympathetic activation, when epinephrine is released via circulation and from sympathetic nerve terminals into the skin. Epinephrine infusion may be considered a more realistic way of mimicking the adrenomedullary response during anxiety than the peripheral administration of adrenergic agonists. To study effects of epinephrine in isolation, subjects had to be prevented from becoming anxious due to the arousal or bodily sensations associated with epinephrine. Therefore, subjects were selected who scored below normal population means on measures of fear of bodily sensations, in whom epinephrine is expected to induce only minimal anxiety (Veltman, 1995).

Even when anxiety is not induced, however, it is possible that the arousal associated with epinephrine infusion increases pain through attribution. Based on attribution theory (Reisenzein, 1983; Nisbett \& Schachter, 1966), it has been argued that subjects may falsely interpret arousal induced by anxiety as part of the pain (Weisenberg et al., 1984; Dougher, Goldstein \& Leight, 1987; Al Absi \& Rokke, 1991). Data from Rogers \& Deckner (1975) suggest that epinephrine affects subjective measures through attribution in the absence of distracting stimuli onlly. This implies that, when attention is distracted from pain, subjective pain is less likely to be increased by the attribution of arousal. As demonstrated in several studies (Arntz, Dreessen \& Merckelbach, 1991; Arntz, Dreessen \& de Jong, 1994; Arntz \& de Jong, 1993; Janssen \& Arntz, 1996), attentional focus is an important mediator in the influence of anxiety on pain: anxiety has no effect when controlling for attentional focus, while attention on pain leads to a pain increase compared to distraction from pain. To distinguish between possible attributional effects and more peripheral (phy siological) effects of epinephrine, the focus of attention was manipulated in the present study. Also, manipulating attention allows to investigate the relative contribution of epinephrine and attentional mechanisms in the influence of anxiety on pain.

Since different pain measures may be differentially affected by physiological changes, three pain measures were used: subjective pain due to electrical stimulation, heat pain threshold, and pressure pain threshold. Attentional focus was manipulated within electrical stimulation only. The effect of epinephrine on heat pain threshold was examined on both normal and sensitized skin. This was necessary because previous research often found effects on sensitized skin, but not always on normal skin. Therefore, the absence of an effect on normal skin would not exclude the possibility of hyperalgesic effects of systemic epinephrine on sensitized skin. Following Drummond (1995), capsaicin was used to temporarily sensitize the skin as a model of inflammation, after which pain threshold for heat pain was measured on both normal and sensitized skin. In 
contrast to Drummond, who used doses of norepinephrine that do not naturally occur in human skin, we aimed to demonstrate effects on pain sensitivity of epinephrine levels in blood comparable to those seen during anxiety.

Thus, epinephrine infusion, as compared to placebo, was expected to increase subjective and physiological pain responses due to electrical pain stimulation in a dose-dependent way. Independent of this effect, attention to pain was expected to increase pain with respect to distraction. However, if attribution plays a role the effect of epinephrine versus placebo would be expected to be larger in the attention than in the distraction condition. Furthermore, epinephrine infusion was hypothesized to decrease pain threshold for heat and pressure pain with respect to placebo in a dose-dependent way. However, if the influence of epinephrine on pain is restricted to sensitized skin, only in capsaicin-treated skin a decrease in heat pain threshold would be expected.

\section{Method}

\section{Subjects}

Twenty-four subjects, 12 female and 12 male, were recruited from the student population, receiving a small remuneration for participating. Selection criteria were a score below normal population means on Chambless et al.'s (1984) Bodily Sensations Questionnaire 1 (lower than 27, mean 22.2, SD 4.2) and 2 (lower than 31, mean 25.6, SD 4.8) and on Reiss et al.'s (1986) Anxiety Sensitivity Index (lower than 18, mean 5.8, SD 3.1). Subjects scored low (mean 30.5, SD 7.2, 3rd decile) on Trait Anxiety (STAl, Spielberger et al. 1970). Exclusion criteria were cardiovascular disease, high blood pressure and diabetes. Ages ranged between 18 and 34 (mean age 23, SD $4 \mathrm{yr}$ ). All subjects signed an informed consent form after being informed about the procedure, which was approved by our Medical Ethics Committee.

\section{Materials}

The BSQ3 (Chambless et al., 1984) was used to assess subjects' bodily sensations during the experiment. Subjective pain, anxiety and amount of attention paid to the pain stimulus were rated on $100-\mathrm{mm}$ visual analogue scales (VASs) ranging from "not at all ..." to "extremely...". A video tape recording of a documentary about a glass factory was shown before the experiment as a neutral induction, while an episode of L.A.Law was used as a distractor during the experiment.

\section{Apparatus and physiological recording}

Apparatus was placed in an adjacent room. An electrical pain stimulus was delivered at the left ankle, rubbed with SkinPure (Nihon Kohden) to reduce skin resistance, via two $8 \mathrm{~mm} \mathrm{Ag-AgCl}$ SensorMedics electrodes (distance $2.5 \mathrm{~cm}$ ) filled with Spectra 360 electrode gel. Electrical stimulation consisted of $20 \mathrm{msec}$ rectangular pulses of alternating current $(25 \mathrm{~Hz})$, rising to the preselected level within $0.5 \mathrm{sec}$, remaining constant for $2 \mathrm{sec}_{\text {, }}$ and returning to zero during $0.5 \mathrm{sec}$. The stimulation produces a stinging sensation, unlike shocks caused by electric fencing or electric mains (Price \& Tursky 1975; Notermans 1966). The heat pain 
stimulus was delivered by a TSA-2001 (Medoc) via a $2 \times 4 \mathrm{~cm}$ thermocouplecontrolled device placed on the dorsal forearm. It started from a holding temperature of $33^{\circ} \mathrm{C}$ increasing by $0.5^{\circ} \mathrm{C} / \mathrm{sec}$. At heal pain threshold, the subject pressed a button and the temperature immediately returned to $33^{\circ} \mathrm{C}$. Temperature could not exceed $50^{\circ} \mathrm{C}$. which regarding the stimulus duration eliminated the risk of burning. Pressure pain was produced by a Forgione-Barber stimulator (Forgione \& Barber, 1971), applying a $1.7 \times 16 \mathrm{~mm}$ plexiglass wedge with a pressure of $62.5 \mathrm{gram} / \mathrm{mm}^{2}$ to the middle phalanx of the second finger of the nondominant hand. This usually begins with a sensation of weight, becoming painful after some time. Pain threshold was measured with a stopwatch.

Heart rate was measured via three disposable electrodes (Meditrace, AgAgcl) connected to the registration apparatus (Picker Schwarzer ED 14). After the skin was cleaned with alcohol, the electrodes were placed just above the sternum, at the left side of the chest, and at the left shoulder as a reference. Interbeat intervals were determined, and changed into heart rate in beats per minute on a second-to-second basis. Heart rate response (HRR) was defined as the mean change in heart rate (in beats per minute) during the 20 sec after the onset of the stimulus, using the mean heart rate during the 10 sec prior to onset as reference. Skin conductance level (SCL) and skin conductance responses (SCRs) were measured by a Beckman Skin Conductance Coupler (type 9844) using the method of constant voltage $(0.5 \mathrm{~V})$. Two SensorMedics electrodes $(8 \mathrm{~mm} \mathrm{Ag-}$ $\mathrm{AgCl})$ were filled with redux creme and, after the skin was cleaned with distilled water, were attached to the medial phalanges of the second and third fingers of the left hand.

\section{Pharmacological manipulation}

For infusion of epinephrine or saline a cathether (Baxter $1.1 / 32 \mathrm{~mm}$ ) was intravenously inserted in the subject's elbow. This was done at least 30 minutes before the first pain ratings. An epinephrine solution $(0.25 \mathrm{mg} / 50 \mathrm{~mL}$ ) was administered in three increasing doses $(20,40$ and $80 \mathrm{ng} / \mathrm{kg} / \mathrm{min}$ ) by an electronic diffusion pump. The highest dose is known to produce epinephrine plasma levels comparable to those reached in highly stressful situations such as speaking before an audience (Dimsdale \& Moss, 1980; Veltman, 1995). To reach a balance between supply and uptake of epinephrine, measurements in each dose were preceded by an adaptation period of 10 minutes. Corresponding doses and times were used for the infusion of saline.

For application of capsaicin an area on the dorsal forearm was cleaned with alcohol. Capsaicin powder (Sigma) was dissolved in 50\% ethanol in water at a concentration of $0.02 \mathrm{M}$ capsaicin $(0.6 \%)$. A $2 \times 2 \mathrm{~cm}$ gauze pad containing 300 ul of this solution was applied to the prepared skin. The pad was covered with plastic tape to retard evaporation. Thirty minutes later the gauze pad was removed and the treated skin was washed with soap and water.

\section{Design}

Within subjects, pharmacological condition (epinephrine vs. placebo) and dose (low, medium, high) were varied, resulting in six experimental conditions. A pretest with saline was done before the experimental conditions. To control for 
order effects, order of pharmacological condition was varied between subjects, blind to both subject and experimenter. Dependent variables were subjective pain, HRR and SCR as a result of the electrical stimulus, pain threshold for the heat stimuius (in ${ }^{\circ} \mathrm{C}$ ), and pain threshold for the pressure stimulator (in sec). The BSQ3 and the VASs on anxiety and attention were used as manipulation check. During electrical stimulation, the attentional focus (attention on pain vs, attention distracted by video) was varied as an additional within-subjects variable, resulting in a $2 \times 3 \times 2$ design. Heat pain threshold was measured on capsaicin-treated ws. untreated skin, also resulting in a $2 \times 3 \times 2$ design. For pressure pain a $2 \times 3$ design was employed. The arm treated with capsaicin was counterbalanced across pharmacological order, as were the order of attention condition and the order of capsaicin condition (linked, see Table 1). Males and females were evenly distributed across conditions.

Table 1. The variables controlled for in the design: order of pharmacological manipulation (epinephrine and placebo), arm treated with capsaicin (left vs. right), order of heat pain stimulation (capsaicin and control arm), and order of altention condition (attention and distraction).

\begin{tabular}{lllll}
\hline Pharmacological order & Capsaicin arm & Heat pain order & Attention order & \# \\
\hline \multirow{3}{*}{ Epinephrine/ Placebo } & Left & Caps/Con & Att/ Dis & 3 \\
& & Con/Caps & Dis/Att & 3 \\
& Right & Caps/Con & Att/ Dis & 3 \\
& & Con/Caps & Dis/Att & 3 \\
\hline \multirow{2}{*}{ Placebo/ Epinephrine } & Left & Caps/Con & Att/ Dis & 3 \\
& & Con/Caps & Dis/Att & 3 \\
& Right & Caps/Con & Att/ Dis & 3 \\
& & Con/Caps & Dis/Att & 3 \\
\hline
\end{tabular}

\section{Procedure}

Upon arrival, subjects were medically tested and weighed by a doclor. They were informed about the infusion of epinephrine, known to be mildly activating but harmless. They were told that the experimenters did not know when epinephrine or saline was administered and that their main interest was on the physiological responses to stimuli.

After capsaicin was applied to the skin, the catheter was inserted and the electrodes were fastened. Subjects received instructions about the procedure, the stimuli and the VASs. When capsaicin was removed, the electrical stimulation level was individually established. The level was increased in steps of $0.2 \mathrm{~mA}$ until pain threshold level (anchored at $25 \mathrm{~mm}$ on the VAS), and then increased until $70 \mathrm{~mm}$ on the VAS. This level was maintamed during the experiment. Mean threshold level was $1.3 \mathrm{~mA}$ (SD 1.1), mean level used in the experiment was 4.5 MA (SD 2.5).

When capsaicin no longer produced spontaneous pain, saline was 
administered, while a neutral video about a glass factory was shown to make subjects feel as much at ease as possible. After 8 minutes, subjects scored the

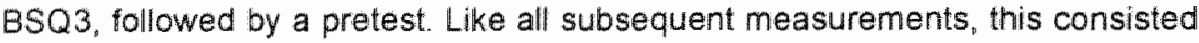
of:

Electrical stimulation: Two electrical stimuli were given with the instruction to concentrate as much as possible on the pain stimulus, and two with the instruction to watch an L.A.Law episode. Each series of two stimuli was announced 1 minute in advance, the interval between the two stimuli varied between 20 and 35 sec. After each series subjects scored the 3 VASs.

Heat pain: A series of four heat stimuli, $15 \mathrm{sec}$ apart, was given on both the capsaicin-treated and the untreated arm. Subjects were asked to concentrate on the heat stimulus, signalled by a beep, and press the button when heat pain threshold was reached.

Pressure pain: The second finger of subjects' non-dominant hand was placed under the pressure stimulator. With intervals of 20 sec. subjects were asked to indicate pressure on a scale of 0 to 25, 0 meaning "no feeling at all", 25 meaning pain threshold. As soon as pain threshold was reached, the pressure was taken off and the time on the stopwatch was recorded.

Immediately after the pretest, the infusion of either epinephrine or saline in three increasing doses was started. The $10 \mathrm{~min}$. adaptation period that preceded measurement in each dose, during which subjects were allowed to read magazines, was interrupted at the end by the scoring of the BSQ3. After each adaptation period, the pain measurements were started. When the highest dose condition was over, the infusion pump was switched to the other pharmacological condition. After the last condition, the subject was debriefed and thanked for participation.

Table 2. Means (SD) of the variables used as manipulation check, displayed for each attention condition and for each dose of placebo or epinephine.

\begin{tabular}{lccccccc}
\hline & \multicolumn{3}{c}{ Placebo } & \multicolumn{3}{c}{ Epinephirine } \\
\cline { 2 - 8 } Dose (ng $/ \mathrm{kg} / \mathrm{min})$ & 20 & 40 & 80 & 20 & 40 & 80 \\
\hline ESQ3 & & $19(3.2)$ & $19(2.3)$ & $18(2.3)$ & $19(2.5)$ & $22(5.9)$ & $23(6.8)$ \\
\hline HR (bpm) & Att & $68(8.4)$ & $69(7.4)$ & $69(9.4)$ & $70(8.4)$ & $71(7.8)$ & $77(8.5)$ \\
& Dis & $69(8.4)$ & $67(7.7)$ & $67(9.0)$ & $71(9.3)$ & $72(8.5)$ & $78(9.9)$ \\
\hline SCL/pre (\%) & Att & $99(25)$ & $104(26)$ & $107(25)$ & $105(20)$ & $103(19)$ & $105(28)$ \\
& Dis & $100(19)$ & $106(23)$ & $107(23)$ & $106(19)$ & $104(21)$ & $106(29)$ \\
\hline \multirow{2}{*}{ Anxiety VAS } & Att & $27(25)$ & $22(22)$ & $21(22)$ & $30(22)$ & $36(25)$ & $35(26)$ \\
& Dis & $20(21)$ & $16(18)$ & $14(16)$ & $21(20)$ & $25(24)$ & $24(24)$ \\
\hline \multirow{2}{*}{ Attemtion VAS } & Att & $74(16)$ & $74(19)$ & $75(17)$ & $76(18)$ & $75(16)$ & $81(12)$ \\
& Dis. & $19(18)$ & $25(20)$ & $15(15)$ & $19(20)$ & $22(22)$ & $23(23)$ \\
\hline
\end{tabular}




\section{Results}

\section{Manipulation check (see Table 2)}

Infusion of epinephrine led to higher scores than placebo on the BSQ3 $(F(1,22)$ $=16.9, p<0.001$ ), in a dose-dependent way (linear trend, $t(22)=4.4, p<0.001$ ), as well as to higher mean heart rates $(F(1,22)=28.1, p<0.001)$, also dosedependently (linear trend, $t(22)=8.5, p<0.001$ ), but not to heightened skin conductance levels $(F(1,22)=0.03$, n.s. $)$. Epinephrine dose-dependently led to higher anxiety VAS scores $(F(1,22)=17.4, p<0.001$; linear trend, $t(22)=2.2$, $p<0.05$ ), although anxiety seemed more influenced by attention manipulation, scores being lower in the distraction than in the attention condition $(F(1,22)=$ $23.2, p<0.001$ ). Finaliy, the manipulation of attention was successful: attention to pain was rated much higher in the attention than in the distraction condition $(F(1,22)=156.3, p<0.001)$, whereas it was not influenced by pharmacological manipulation $(F(1,22)=1.9$, n.s. $)$.

Table 3. Means (SD) of the experimental variables, displayed for each attention condition and for each dose of placebo or epinephrine.

\begin{tabular}{|c|c|c|c|c|c|c|c|}
\hline \multirow{2}{*}{\multicolumn{2}{|c|}{ Dose (ng $/ \mathrm{kg} / \mathrm{min}$ ) }} & \multicolumn{3}{|c|}{ Placebo } & \multicolumn{3}{|c|}{ Epinephrine } \\
\hline & & \multirow{3}{*}{$\begin{array}{c}20 \\
59(24) \\
50(23)\end{array}$} & \multirow{3}{*}{$\begin{array}{c}40 \\
56(22) \\
45(24) \\
\end{array}$} & \multirow{3}{*}{$\begin{array}{c}80 \\
55(26) \\
43(27)\end{array}$} & \multirow{3}{*}{$\begin{array}{c}20 \\
58(23) \\
49(26)\end{array}$} & \multirow{3}{*}{ 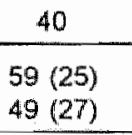 } & \multirow{3}{*}{ 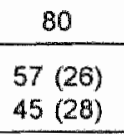 } \\
\hline Pain VAS & Att & & & & & & \\
\hline & Dis & & & & & & \\
\hline \multirow[t]{2}{*}{$\sqrt{S C R} /$ pre $(\%)$} & Att & $0.8(0.4)$ & $0.9(0.3)$ & $0.8(0.4)$ & $0.9(0.3)$ & $0.8(0.3)$ & $0.6(0.3)$ \\
\hline & Dis & $0.9(0.4)$ & $0.9(0.4)$ & $0.7(0.4)$ & $0.8(0.3)$ & $0.7(0.4)$ & $0.6(0.3)$ \\
\hline \multirow[t]{2}{*}{ HRR (bpm) } & Att & $1.2(2.8)$ & $1.5(2.9)$ & $2.7(2.4)$ & $2.5(3.7)$ & $2.3(2.9)$ & $1.9(2.4)$ \\
\hline & Diss & $0.5(1.9)$ & $1.4(1.6)$ & $1.4(1.9)$ & $4.2(2.8)$ & $1.3(2.2)$ & $0.5(2.9)$ \\
\hline \multirow{2}{*}{$\begin{array}{l}\text { Heat pain } \\
\text { threshold }\left({ }^{\circ} \mathrm{C}\right)\end{array}$} & Con & $4.4 .8(3.1)$ & $44.6(3.2)$ & $44.6(3.3)$ & $44.2(3.4)$ & $44.0(3.3)$ & $43.9(3.0)$ \\
\hline & Cap & $40.1(3.2)$ & $38.9(3.1)$ & $38.5(3.3)$ & $38.9(2.9)$ & $37.8(2.6)$ & $36.5(2.1)$ \\
\hline \multicolumn{2}{|l|}{$\begin{array}{l}\text { Pressure pain } \\
\text { threshold (sec) }\end{array}$} & $33(32)$ & $31(35)$ & $31(35)$ & $35(23)$ & $35(33)$ & $29(27)$ \\
\hline
\end{tabular}

\section{Electrical stimulation}

A group difference existed between subjects with a different order of pharmacological manipulation, electrical stimulation being rated as more painful by those receiving epinephrine first $(F(1,22)=5.8, p<0.05)$. Since this difference was already present at pretest $(F(1,22)=9.2, p<0.01)$, it cannot be attributed to experimental manipulations. The effect on subjective pain (see Figure 1 and Table 3) of epinephrine versus placebo was not significant $(F(1,22)=0.8$, n.s. The overall effect of dose, independent of pharmacological condition (linear trend $t(22)=-2.6, p<0.05)$, indicates that there was a habituation over time. However, there was a marginally significant interaction between pharmacological condition and quadratic dose trend $(t(22)=2.0, p=0.06)$, suggesting that, on top of this 
habituation, epinephrine had a quadratic dose effect. When all data points were transformed into their deviation from a hypothetical line drawn between pretest and final measurement as an estimate of habituation, epinephrine turned out to have a marginally significant pain-increasing effect compared to placebo $(F(1,22)$ $=3.0, p=0.10)$. Attention manipulation strongly affected subjective pain $(F(1,22)$ $=21.0, p<0.001)$, with lower ratings in the distraction than in the attention condition. There was no interaction between pharmacological and attention condition $(F(1,22)=0.01$, n.s. $)$.

Heart rate response to electrical stimulation was not influenced by epinephrine $(F(1,22)=0.5, n . s$.$) , but distraction decreased HRR compared to attention on$ pain $(F(1,22)=7.4, p<0.05)$. Although epinephrine slightly increased HRR in the attention condition, the interaction between pharmacological and attention condition did not reach significance $(F(1,22)=1.8, n . s$. $)$.

Skin conductance response was influenced by pharmacological manipulation, but, contrary to expectation, it was inhibited by epinephrine $(F(1,22)=10.0, p<$ 0.05 ), slightly depending on dose $(F(2,44)=2.4, p=0.10)$. There was no attention effect on $\operatorname{SCR}(F(1,22)=0.2$, n.s.), nor an interaction between pharmacological and attention manipulation $(F(1,22)=2.0$, n.s.).

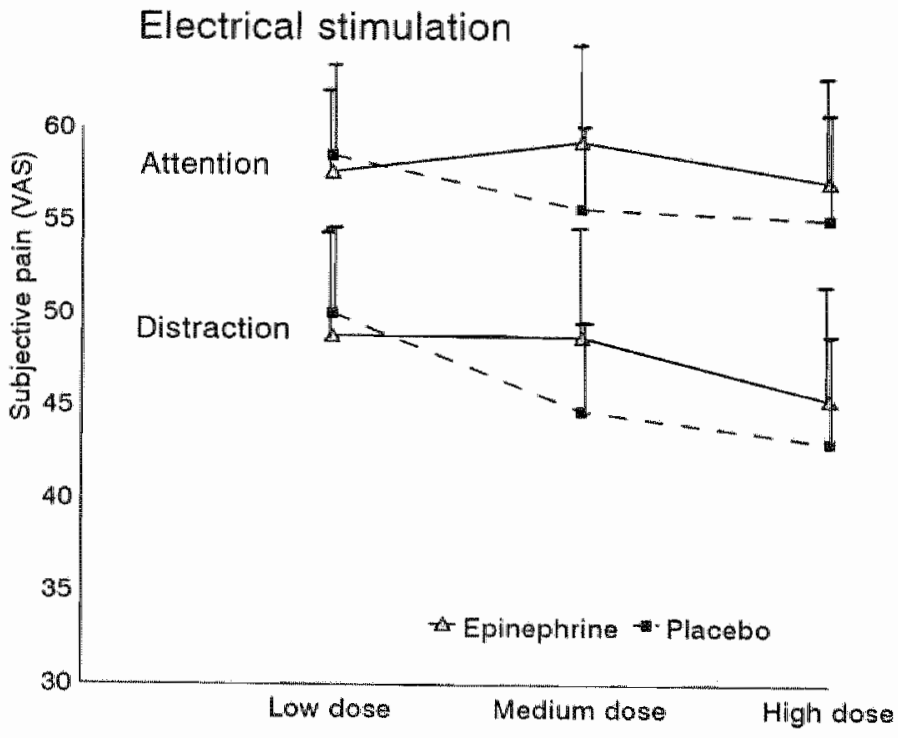

Figure 1. Subjective pain due to electrical stimulation, displayed for each attention condition and dose of epinephrine and placebo. 


\section{Skin conductance response}

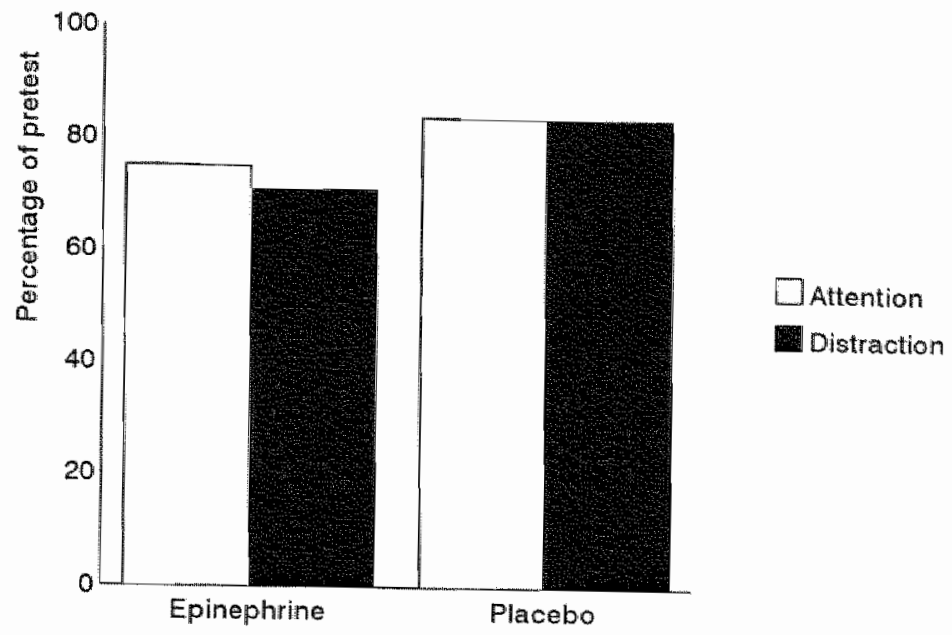

Heart rate response

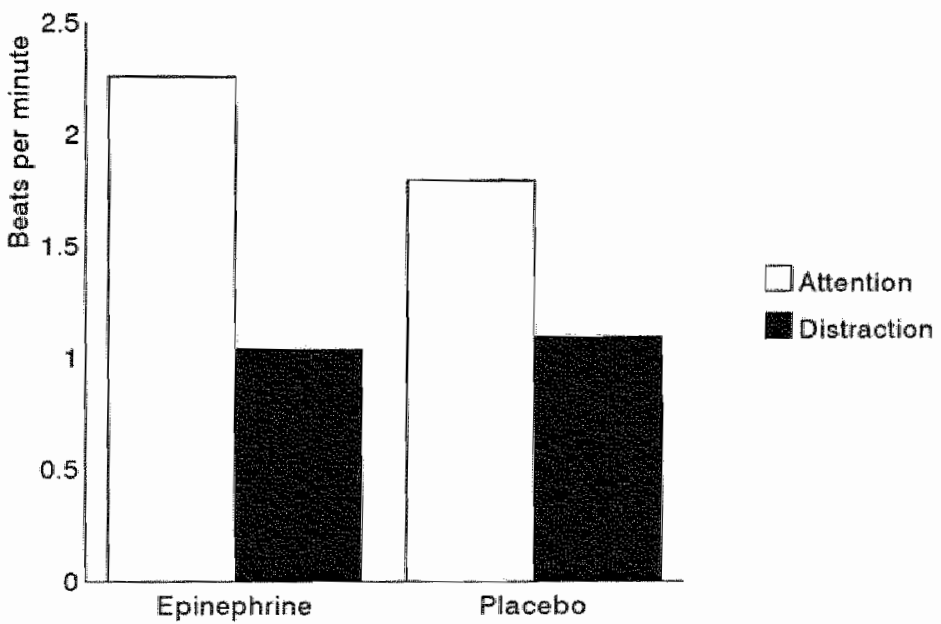

Figure 2. Skin conductance response and heart rale response due to electrical stimulation, displayed for each pharmacological (averaged over dose) and attention condition. 


\section{Heat pain}

It is clear from Figure 3 that capsaicin-treated skin showed lower heat pain thresholds than normal $\operatorname{skin}(F(1,22)=136.6, p<0.001)$. A main increase in sensitivity was observed over time (linear trend, $t(22)=6.1, p<0.001$ ), which was stronger on capsaicin-treated skin, as shown by the interaction between time and capsaicin condition (linear trend, $t(22)=-5.4, p<0.001$ ). Further, epinephrine decreased heat pain thresholds compared to placebo $(F(1,22)=22,8, p<0.001)$, although this effect was not dose-dependent $\langle F(2,44)=1.4$, n.s.). The epinephrine effect was strongest on capsaicin-treated skin, as shown by the interaction between pharmacological manipulation and capsaicin condition $(F(1,22)=4.6, p<0.05)$. However, when analyzed separately, the effect on capsaicin-treated skin $(F(1,22)=20.5, p<0.001)$ and normal $\operatorname{skin}(F(1,22)=6.1$, $p<0.05)$ both reached significance.

\section{Heat pain threshold}

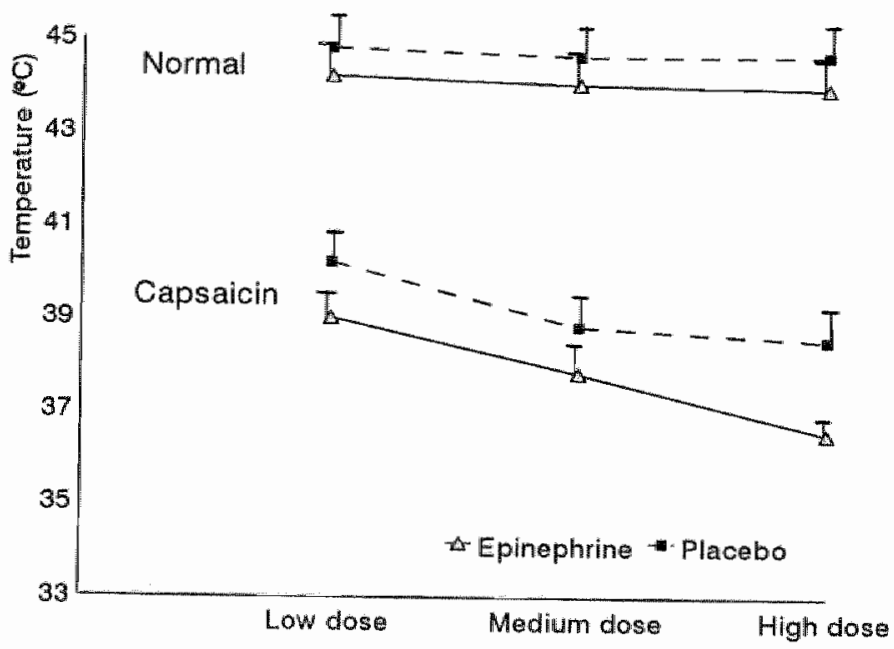

Figure 3. Heat pain threshold for each dose of epinephrine and placebo, in both normal and capsaicin-treated skin.

\section{Pressure pain}

There was no effect of pharmacological manipulation on pressure pain threshold $(F(1,22)=0.5, n . s$.$) , nor an interaction between pharmacological manipulation$ and dose. 


\section{Discussion}

Intravenous infusion of epinephrine turned out to have varying effects on subjective and physiological responses to different pain stimuli. With respect to the habituation shown during placebo, subjective pain due to electrical stimulation showed a slight increase as a result of epinephrine, although heart rate response did not and skin conductance response was even inhibited. Pain threshold for heat pain stimulation showed the expected decrease during epinephrine infusion, both in skin pre-treated with capsaicin and (although smaller) in normal skin. However, there was no effect of epinephrine infusion on pressure pain threshold, perhaps due to the large within-subject variance on this measure. While the observed effects are small, the conclusion seems justified that in some circumstances, especially when tissue is sensitized or damaged, a rise in epinephrine plasma level leads to an increase in pain.

Although epinephrine does not cross the blood-brain barrier and may be expected to have only peripheral effects, it cannot be excluded that more central patthways were triggered indirectly. Firstly, anxiety levels were slightly raised during infusion of epinephrine, which may have caused endogenous opioids to be released. However, opioid release would be expected to inhibit rather than increase pain. Secondly, blood pressure may have been slightly elevated by epimephrine (Weiner, 1985). Again, this would not explain a pain increase, since, if anything, hypertensive patients tend to have lower pain sensitivity than normal subjects (Al-Absi, Buchanan \& Lovallo, 1996). Thirdlly, epinephrine infusion may indirectly (perhaps through hypertension) have triggered an inhibition of centrall sympathetic activation through autoregulatory mechanisms. However, this would rather be expected to reduce epinephrine rellease from sympathetic nerve terminals, underestimating the effect of infused epinephrine and perhaps camouflating a dose effect of epinephrine.

It may come as a surprise that skin conductance responses showed an inhibition during epinephrine infusion, as skin conductance is known to be predominantly sympathetically mediated (Dawson, Schell \& Filion, 1990). The level of skin conductance was not influenced by epinephrine (see Table 2), ruling out the possibility that the reduction in phasic responses was due to higher levels of tonic activation during epinephrine infusion. However, skin conductance is primarily associated with central sympathetic activation (Dawson, Schell \& Filion, 1990), which, through a feedback mechanism, may have been inhibited by the rise in peripheral epinephrine, leading to decreased responses.

Although an answer is beyond the scope of this paper, the question should be addressed through what mechanisms peripheral epinephrine may have caused hyperalgesia. It has been argued that vasoconstriction in the skin mediates changes in pain sensitivity when studying effects of sympathetic activation (Wiesenfeld-Hallin \& Hallin, 1984). However, this could not explain the entire effect, since peripheral norepinephrine and arterial occlusion proved to have independent effects on heat pain sensitivity (Drummond, 1996). Animal studies have shown that sympathetic stimulation alone does not excite nociceptors (Shea \& Perl, 1985; Barasi \& Lynn, 1986). However, after receptive field sensitization or peripheral nerve injury, nociceptor discharge is markedly facilitated by 
sympathetic stimulation or local norepinephrine injection (Roberts \& Elardo, 1985; Hu \& Zhu, 1989; Sato \& Perl, 1991). Perhaps repeated testing also results in some sensitization of receptive fields, which would explain why adrenergic agonists induced a small heat hyperalgesia in nomal skin in the present study and in Drummond (1996) and Choi \& Rowbotham (1997). Recent evidence suggests that both stimulus-evoked and ongoing pain following capsaicin treatment depend on a-adrenergic receptors (Kinnman, Nygărds, Hansson, 1997). Epinephrine infusion may have increased stimulus-evoked pain as well as reevoked capsaicin-induced ongoing pain thereby increasing responsiveness to epinephrine in capsaicin-treated as compared to normal skin. However, while heat hyperalgesia, seen after capsaicin treatment, most likely represents sensitization at nociceptor level, hyperalgesia for some types of mechanicall stimuli seems to involve central sensitization (Koltzenburg, Lundberg, \& Torebjôrk, 1992; Kilo et al., 1994). Perhaps associated with this, there is evidence that not all types of pain are equally affected by adrenergic antagonists: after phentolamine injection, brushing-evoked pain areas, but not pinprick pain areas, had lessened (Liu et al., 1996). Such a dissociation may have accounted for the differential effects on pain measures. So far, no real consensus has been reached on the exact way in which the sympathetic nervous system influences pain, but more detailed knowledge on the underlying mechanisms may come from future studies on sympathetically maintained pain.

While effects of epinephrine on pain were demonstrated, the effect in normal skin was only very small. With electrical stimulation, the evidence for an epinephrine-induced hyperalgesia was only marginally significant, whereas the effect of attentional focus was much stronger, with attention on the electrical stimulus leading to increases in subjective pain and heart rate response as compared to distraction. This is consistent with previous studies showing strong effects of attentional focus and no effect of anxiety on the experience of electrical pain stimuli (Arntz, Dreessen \& Merckelbach, 1991; Arntz, Dreessen \& de Jong, 1994; Arntz \& de Jong, 1993; Janssen \& Arntz, 1996). The absence of an attention effect on skin conductance response may be explained by the heightened unexpectedness of the stimulus when one is distracted, any inhibiting effects of distraction being masked by an increase in response due to the unpredictability of the stimulus (Arntz, Dreessen \& de Jong, 1994; Janssen \& Arntz, 1996). Although only measured with electrical stimulation, the absence of interactive effects between attention manipulation and epinephrine infusion makes it unlikely that any effects of epinephrine infusion on pain depended on the focus of attention or on the attribution of arousal to the pain.

Assuming that in an anxious state pain is increased due to epinephrine release, are the effects large enough to have important implications for real-life situations? Epinephrine plasma levels comparable to those during anxiety only led to minor changes in pain sensitivity for electrical stimulation and heat pain in normal skin. However, the heat pain data suggest that when skin is damaged, epinephrine may importantly affect pain perception and behaviour. Increased sensilivity for pain may protect the individual from (further) damage in dangerous and anxiety-inducing situations. In normal skin, however, although only assessed with electrical stimulation, attention seems to have by far the largest impact, 
potent enough to overrule effects of epinephrine on pain. This may be important in life-threatening situations, when escaping from danger has higher priority than preventing tissue damage. In contrast to Bolles and Fanselow's hypothesis that endogenous opioids are released during anxiety to facilitate fight or flight behaviour (Bolles \& Fanselow, 1980), the phenomenon of stress-induced analgesia may be restricted to situations in which one is helpless and not in control of the situation (Henry, 1990; Janssen \& Arntz, 1997). Several mechanisms may simultaneously influence pain perception, some increasing and some inhibiting pain, the relative contribution of each effect depending on how the situation is perceived. Sometimes, however, these mechanisms do not seem very adaptive. In patients experiencing acute pain in clinical settings, anxiety for pain may often result in an attentional allocation towards the pain as well as in enhanced levels of epinephrine, both leading to increased pain sensitivity. The present findings suggest that, when it is possible to distract attention, this could have larger effects on pain than reducing sympathetic activation due to anxiety. However, when anxiety for pain is too high to distract attention from the painful stimulus, reducing anxiety and sympathetic activation may become relatively more effective. 



\section{Does anxiety lead to less distraction from pain?}

\section{Summary}

11. was tested whether pain-irrelevant anticipatory anxiety increasies pain as compared to no anxiety due to the automatic allocation of attention to pain. Thirty-two high and 32 low trait socially anxious female students recived painful electrical stimulation at the ankle, seated in elther a stimulus-rich or a stimulus-poor environment. Before stimulation, half of the subjects in each group were told they were supposed to give an oral presentation, which was expected to induce a state of anxiety in the high trait socially anxious group. Non anxious subjects were hypothesized to show lower pain ratings in the stimulus-rich than in the stimulus-poor environment because of distraction from pain. Subjects with anticipatory anxiety were expected to profit less from being in a distracting environment "because their attention would automatically be focused on the pain. However, anxiety menipulation appeared unsuccessful: irrespective of experimental condition, high trait socially anxious subjects wene more anxious in the experimental situation than low trait socially anxious subjects. Unexpectedly, only the latter group showed an effect of anxiety induction on measures of anxiety. Because of the unsuccessfull manipulation of anxiety, the hypothesis that anxiety increases pain by the automatic allocation of attention to pain could not be tested. However, it is indirectly supported by the finding that high trait socially anxious subjects showed slightly higher ratings of pain and attention to pain, and less memory for meutral stimuli in the environment than low trait socially anxious subjects.

\section{Introduction}

Anxiety and experienced pain intensity have often been found to correlate, in clinical as well as in experimental situations (Volicer, 1978; Dougher, 1979; Malow, West \& Sutker, 1989; Passchier et al., 1992). On the other hand, there are situations in which anxiety seems to have pain-inhibiting effects (Bolles \& Fanselow, 1980; Carter, McNeill \& Reed, 1991; Janssen \& Arntz, submitted). While many researchers studied the influence of induced anxiety on pain perception, no conclusive evidence has been found for an influence of anxiety on pain in either direction. In an attempt to solve this problem, the idea was postulated that anxiety leads to the enhancement of pain only when it is related to the pain (Weisenberg et al., 1984; Dougher, Goldstein \& Leight, 1987; Al 'Absi \& Rokke, 1991). Based on attribution theory (Nisbelt \& Schachter, 1966; Reisenzein, 1983), it was hypothesized that physiological arousal associated with anxiety may increase the experience of pain when it is perceived as part of the pain, which is believed to happen in the case of pain-related anxiety. However, Cornwall \& Donderi (1988) found increases in pain not only after pain-relevant, but also after pain-irrelevant anticipatory anxiety. They concluded that anxiety has perceptually disrupting properties and leads to a reduction in attentional capacity. Therefore, anxiety would result in a tendency to focus attention on the most salient aspects of the situation which may involve the pain. This is consistent with the idea that anxiefy is associated with the automatic allocation of attention 
towards threatening stimuli (Eysenck, 1988). The idea of perceptual disruption during anxiety is also reminiscent of the attentional narrowing theory of Easterbrook (1959), and more recent theorizing that physiological arousal during psychological trauma is associated with increased attention to central details and decreased attention to peripheral details (Christianson, 1992; Wesse \& Merckelbach, 1996). In line with Cornwall \& Donderi (1988), an experiment by Arntz, Dreessen 8 de Jong (1994) showed that not the focus of anxiety, but the focus of attention is a crucial factor in the influence of anxiely on pain. In a series of studies it was demonstrated that attention on pain increases pain compared to distraction from pain, while anxiely does not influence pain when attentional focus is controlled for (Arntz, Dreessen \& Merckelbach, 1991; Arntz \& de Jong. 1993; Janssen \& Arntz, 1996). Altentional focus could provide an explanation for the influence of anxiety on pain in the following way: Usually, pain-related anxiety will result in increased attention towards pain, thereby increasing subjective pain, whereas pain-irrelevant anxiety will result in distraction of attention away from pain towards the object of fear, thereby reducing subjective pain. However, when the object of fear is not immediately present during painful stimulation, anxiety may result in an attentional bias towards the pain being the most salient and threatening stimulus present.

This study aims to investigate whether pain-irrelevant anticipatory anxiety influences pain through the allocation of attention towards pain. The anticipation of an oral presentation, a socially stressfull situation, was used to induce a state of anxiety in socially anxious subjects. Although pain-irrelevant anxiety has been shown to increase pain in the absence of the object of fear (Cornwall 8 Donderi, 1988; Caceres \& Burns, 1997), previous studies did not allow to discriminate between attentionalmechanisms and other factors, such as attribution or sensory changes. In the present study, pain due to electrical stimulation is measured in two situations: in a stimulus-poor and in a stimulus-rich environment (the latter containing neutraf, potentially distracting stimuli). An attentional bias towards pain would only be expected to become manifest when there is competition for attention, since in the absence of other stimuli all subjects, whether they are anxious or not, would focus attention on pain. When potentially distracting stimuli are present, mon-anxious subjects, by focusing attention on neutral stimuli in the environment, may seek more distraction from pain than anxious subjects who are expected to automatically focus attention on the most salient or threatening aspects of the situation.

Thus, in socially anxious subjects without anticipatory anxiety, a decrease in attention on pain and a reduction in pain measures is expected in the stimulusrich compared to the stimulus-poor environment. In socially anxious subjects anticipating an oral presentation, however, no effect of environment, or at least a smaller decrease in the stimulus-rich compared to the stimulus-poor environment than in those without anticipatory anxiety, is expected on attention and pain measures, resulting in an interaction between state anxiety and environment. Because we were also interested in how the manipulation of environment would affect attentional focus and pain perception in subjects who are not anxious at all, the same manipulations were done for subjects low in social anxiety. Since in these subjects anticipatory anxiely is expected to be not 
or hardly induced, attention and pain measures are expected to be reduced in the stimulus-rich compared to the stimulus-poor environment, irrespective of state anxiety manipulation.

\section{Method}

\section{Subjects}

Thirty-two socially anxious and 32 socially non-anxious female undergraduate students were recruited on the basis of their scores on the social phobia subscale of the Fear Questionnaire (FQ, Marks \& Mathews, 1979). A group of 459 students completed the $F Q$, from which subjects belonging to the highest (score $\geq 19$ ) and the lowest percentile (score $\leq 6)$ were approached for participation. Their mean scores on the social phobia FQ were 23.0 (SD 3.1) versus 3.3 (SD 2.3), their mean scores on the Social Phobia Anxiety Inventory (SPAI, Turmer et al., 1989) were 101.2 (SD 24.9) versus 49.3 (SD 18.9). Ages ranged between 18 and 24 (mean age $19.8, S D 1.6 \mathrm{yr}$ ).

\section{Materials}

Painful stimulation was rated on five dimensions using $100 \mathrm{~mm}$ Visual Analogue Scales (VASs), ranging from "nol at all" to "extremely" (painful, tingling, stingy, intense, annoying). Also, anxiety during stimulation and amount of attention focused on the pain stimuli were rated on $100 \mathrm{~mm}$ VASs. Anxilety was induced by telling subjects they had to give an oral presentation, on the basis of which their social abilities would be judged by two independent raters. To provide subjects with potentially distracting but neutral objects, the left side of the laboratory was decorated with the following objects: a poster of a painting by Kandinsky, a callendar picturing well-known buildings in town, a stapier in the form of a blue crocodile, an orange headphone, two cardboard boxes for household and computer apparatus, a yellow mug with a picture of a pig, a giant blue paperclip, a colourful woman magazine cover, a red bag with black and white lines, a pink felt-tip pen, a painted wooden ball, and a yellow checked cloth. As a manipulation check on the amount of time subjects had been engaged in looking at these objects, memory for them was measured by a short interview (Wessel \& Merckelbach, 1996). Free recall was measured by asking subjects to name as many items as they could remember, cued recall by asking them to describe each of the target items in detail (e.g. "What colour was the mug?'). The subject sat facing a video recorder and a television set, while to the right there was just a blank wall.

\section{Apparatus and physiological recording}

Apparatus was placed in an adjacent room. An electrical pain stimulus was delivered at the left ankle, rubbed with SkinPure (Nihon Kohden) to reduce skin resistance, via two $8 \mathrm{~mm} \mathrm{Ag}-\mathrm{AgCl}$ SensorMedics electrodes (distance $2.5 \mathrm{~cm}$ ) filled with Spectra 360 electrode gel. Electrical stimulation consisted of 20 msec rectangular pulses of alternating current, rising to $2.5 \mathrm{~mA}$ within $2 \mathrm{sec}$, remaining constant for $2 \mathrm{sec}_{\text {, }}$ and returning to zero during $2 \mathrm{sec}$. Stimulation produces a stinging sensation, unlike shocks caused by electric fencing, electric mains, etc. 
Heart rate was measured via three disposable electrodes (Meditrace, Ag-AgCl) connected to the registration apparatus (Picker Schwarzer ED 14). After the skin was cleaned with alcohol, the electrodes were placed just above the sternum, at the left side of the chest, and at the left shoulder as a reference. Interbeat intervals were determined, and changed into heart rate un beats per minute on a second-to-second basis. Heart rate response (HRR) was defined as the mean change in heart rate (in beats per minute) during the 20 sec after the onset of the stimulus, using the mean heart rate during the $10 \mathrm{sec}$ prior to onset (HR) as reference. Skin conductance level (SCL) and skin conductance responses (SCRs, defined as the highest deflection in skin conductance starting $1-5$ sec after pain stimulus onset) were measured by a Beckman Skin Conductance Coupler (type 9844) using the method of constant voltage $(0.5 \mathrm{~V})$. Two SensorMedics electrodes ( $8 \mathrm{~mm} \mathrm{Ag-AgCl)} \mathrm{were} \mathrm{filled} \mathrm{with} \mathrm{isotonic} \mathrm{paste} \mathrm{and,} \mathrm{after} \mathrm{the} \mathrm{skin} \mathrm{was}$ cleaned with distilled water, were attached to the medial phalanges of the second and third fingers of the left hand. SCRs were square-roat transformed. Two miniature Sensor-Medics Ag-AgCl electrodes were attached to the skin underneath the eyes only for manipulation (see procedure), nothing was recorded from these.

\section{Design}

A $2 \times 2 \times 2$ analysis of variance was planned, with trait anxiety (high vs. low social anxiety) and anxiety condition (anticipation of presentation vs. no anticipation) as between-subject factors, and environment (stimulus-rich vs. stimulus-poor) as within-subject factor. Dependent variables were subjective pain, heart rate response and skin conductance response as a result of electrical stimulation. VAS ratings of anxiety, attention on pain and credibility, as well as skin conductance level and mean heart rate prior to stimulation, were used as a manipulation check.

\section{Procedure}

Subjects were unaware that they would be asked to perform an oral presentation, but were told that the experimenters were interested in measuring their physiological responses and feelings during pain and other situations. Upon entering, they were seated facing the blank wall, after which electrodes were fastened and electrical stimulation and measurement of physiological responses were explained. It was made clear that electrical stimulation would always be announced in advance. An example of the electrical stimulus was delivered "and pain characteristics, anxiety, and attention on pain were rated as a pretest. Subsequently, subjects were asked to turn their chair towards the television set. The next three minutes, they were shown a video tape recording of a girl giving an oral presentation. While watching, they were asked to rate the girl's performance on a questionnaire, containing items on the ability, characteristics and behaviour of the speaker. After the video, half of the subjects were informed that immediately after the pain test they were expected to give an oral presentation themselves, about a topic they could choose from three cards which would be shown after the pain test. They were told that the presentation would be recorded on video, on the basis of which their social abilities would be judged 
by two independent raters. The other subjects were not told anything and went straight on to the pain test. For the pain test, subjects were turned facing either the blank wall or the possibly distracting neutral objects, in counterbalanced order. Subjects were asked to turn towards the blank wall so that the electrodes beneath their eyes could be plugged into a (loose) cable from underneath the door, while for the other trial the electrodes were plugged into the apparatus to the subject's left. As a rationale for doing the pain test in both positions, subjects were told that two different ways of measuring physiological responses on the skin beneath the eyes were compared. In fact, nothing was measured from these electrodes. In both trials, four pain stimuli were given within wo minutes, with the same intensity and duration as the pretest. After the fourth stimulus, VASs on pain, anxiety and attention on pain were rated, and position was changed. After two pain trials, subjects rated on VASs the credibility of the anxiety induction ("To what extent did you believe, during the pain trials, that you would have to give an oral presentation?'), and the amount of time they had spent thinking about the presentation during each of the two pain trials.

Subsequently, subjects were asked to turn towards the blank wall and were questioned on their memory for the objects in the room. After the memory interview, all subjects were asked to perform a three minute oral presentation as part of another experiment, during which eye blink responses to short loud noises were measured. At the end of the experiment, subjects were debriefed and assured that the video would be erased. None of the subjects guessed the true reason for the presence of the neutral objects or for doing the pain trial in two different positions.

Table 1. Means (SD) of manipulation check variables: expectation of and amount of time spent thinking about the oral presentation, anxiety, attention on pain, skin conductance level (SCL), mean heart rate (HR), and amount of objects remembered during free recall and cued recall.

\begin{tabular}{llllll}
\hline & & \multicolumn{2}{l}{ Low trait social anxiety } & \multicolumn{2}{l}{ High trait social anxiety } \\
\cline { 4 - 7 } & Enwironment & No anticip. & Anticipation & No anticip. & Anticipation \\
\hline \multicolumn{2}{l}{ Expectation (NAS) } & $1.1(1.8)$ & $80.3(23.2)$ & $6.6(12.2)$ & $79.8(12.5)$ \\
Thinking & Poor & $3.3(7.9)$ & $24.3(19.2)$ & $8.6(14.8)$ & $50.3(29.2)$ \\
NAS) & Rich & $3.4(7.9)$ & $28.6(22.4)$ & $6.4(9.8)$ & $45.0(27.7)$ \\
Anxiety & Poor & $24.4(19.7)$ & $30.3(23.9)$ & $44.1(22.1)$ & $44.1(23.4)$ \\
(VAS) & Rich & $24.6(22.5)$ & $23.6(22.2)$ & $38.6(23.4)$ & $42.2(24.3)$ \\
Attention & Poor & $48.4(26.9)$ & $59.3(21.1)$ & $61.6(24.1)$ & $63.3(20.1)$ \\
(VAS) & Rich & $40.6(32.9)$ & $47.6(28.5)$ & $51.8(19.1)$ & $62.7(22.1)$ \\
SCL & Poor & $5.81(1.83)$ & $8.12(2.15)$ & $7.19(1.89)$ & $7.69(1.76)$ \\
(HSiemens) & Rich & $5.89(1.91)$ & $7.92(2.05)$ & $7.05(1.84)$ & $7.61(1.86)$ \\
HR & Poor & $79.3(10.7)$ & $82.6(10.0)$ & $80.5(11.0)$ & $80.4(15.0)$ \\
(bpm) & Rich & $78.3(10.2)$ & $81.4(11.5)$ & $80.7(10.2)$ & $78.2(15.7)$ \\
Free Recall & & $3.5(1.5)$ & $3.6(2.2)$ & $2.8(1.8)$ & $2.8(1.7)$ \\
Cued Recall & & $6.2(2.3)$ & $6.9(3.8)$ & $4.7(2.7)$ & $5.3(2.4)$ \\
\hline
\end{tabular}




\section{Results}

\section{Manipulation check (see Table 1)}

When asked to what extent they expected having to give an oral presentation, subjects in the anticipation and no anticipation condition gave mean VAS ratings of 80 and 4 respectively (Mann-Whitney chi-square $=-6.9 ; p<0.001$ ). In the anticipation condition, when asked about the amount of time during pain stimulation they had been thinking about the presentation, high trait socially anxious subjects gave higher ratings than low trait socially anxious subjects $(F(1,62)=4.69 ; p<0.05)$. Also, high trait social anxiety was associated with significantly higher anxiety ratings during pain stimulation than low trait anxiety $(F(1,60)=10.3 ; p<0.005)$. Strangelly, anxiety ratings were not significantly influenced by anxiety condition $(F(1,60)=0.17 ;$ n.s. $)$, nor was there an interaction between trait anxiety and anxiety condfition $(F(1,60)=0.0 ; n$.s.). Skin conductance level was not influenced by trait anxiety $(F(1,60)=0.91$; n.s.), but was significantly increased in the anticipation as compared to the no anticipation condition $(F(1,60)=8.24 ; p<0.01)$. Paradoxically, however, this was only apparent for low trait anxious subjects, resulting in a marginally significant interaction between trait anxiety and anxiety condition $(F(1,60)=3.04 ; p=0.09)$. Mean heart rate in the $10 \mathrm{sec}$. before stimulation was not influenced by either trait anxiety $(F(1,57)=0.03 ;$ n.s. $)$ or anxiety condition $(F(1,57)=0.05$; n.s.).

The manipulation of attention by environment was successful: attention on pain was rated lower in the stimulus-rich than in the stimulus-poor environment $(F(1,60)=7.8 ; p<0.01)$. Anxiety condition did not significantly influence attention on pain $(\mathbb{F}(1,60)=1.9 ; n . s)$, but trait anxiety had a marginally significant effect, with more attention on pain among the high trait anxious subjects $(F(1,60)=3.8$; $p=0.06$ ). Although in the expected direction (a decrease in attention on pain in the stimulus-rich compared to the stimulus-poor environment, except for high trait anxious subjects in the anticipation condition), the interaction between trait anxiety, anxiety condition and environment did not reach significance $(F(1,60)=$ $1.5 ; n . s$.$) , nor did the interaction between trait anxiety and environment \langle F(1,60)$ $=0.7$; n.s.). Anxiety condition did not influence memory measures $(F(1,60)=0.4$; n.s.), but high trait anxious subjects remembered less than low trait anxious subjects $(F(1,60)=4.5 ; p<0.05)$, without interaction between trait anxiety and anxiety condition $(F(1,60)=0.01 ;$ n.s. $)$.

Table 2. Means (SD) of pain measures: subjective pain, skin conductance response (SCR) and heart rate response (HRR), displayed for each experimental condition.

\begin{tabular}{|c|c|c|c|c|c|}
\hline & \multirow[b]{2}{*}{ Environment } & \multicolumn{2}{|c|}{ Low trait social anxiety } & \multicolumn{2}{|c|}{ High trait social anxiety } \\
\hline & & No anticip. & Anticipation & No anticip: & Anticipation \\
\hline Pain & Poor & $43.1(20.6)$ & $57.8(19.4)$ & $54.8(15.1)$ & $58.6(14.2)$ \\
\hline (VAS) & Rich & $42.0(20.4)$ & $55.5(16.0)$ & $55.2(16.0)$ & $59.9(15.9)$ \\
\hline SCR & Poor & $0.80(0.32)$ & $0.81(0.44)$ & $0.70(0.31)$ & $0.68(0.32)$ \\
\hline (WuSiemens) & Rich & $0.88(0.30)$ & $0.79(0.46)$ & $0.77(0.24)$ & $0.67(0.24)$ \\
\hline HRR & Poor & $-0.14(1.50)$ & $-1.08(1.55)$ & $-0.76(2.25)$ & $-0.96(2.06)$ \\
\hline (bpm) & Rich & $0.45(2.57)$ & $0.29(1.81)$ & $-0.09(2.24)$ & $-0.37(2.56)$ \\
\hline
\end{tabular}


Pain measures (see Table 2)

Factor analysis showed that the five dimensions of pain rated on VAS scales in both the stimulus-poor and the stimulus-rich environment loaded on one factor (explained variances respectively 62 and $63 \%$ ). Therefore, the mean of these ratings was used as a measure of subjective pain (Cronbach alpha 0.84 and 0.85 ). This composite pain score proved higher in the anticipation than in the no anticipation condition (Figure $1 ; F(1,60)=4.8, p<0.05$ ). Although this effect was only apparent in the low trait anxious subjects (see Figure 1), the interaction between trait anxiety and anxiely condition was not significant $(F(1,60)=1.35$; n.s.). High trait anxiety was associated with slightly higher pain ratings than low trait anxiety $(F(1,60)=3.2 ; p=0.08)$. Pain ratings did not show a main effect of environment $(F(1,60)=0.1 ; n . s$.$) , nor the hypothesized threeway interaction$ between trait anxiety, anxiety condition and environment $(F(1,60)=0.3 ; \mathrm{n.s}$.).

Skin conductance response was not significantly influenced by anxiety condition $(F(1,60)=0.43 ; n . s$. $)$ or trait anxiety $(F(1,60)=2.36 ; n . s$.$) , nor by$ environment $(F(1,60)=2.18 ; n . s$. $)$. Heart rate response was not influenced by anxiety condition $(F(1,60)=0.37$; n.s. $)$ or trait anxiety $(F(1,60)=0.64 ; n$.s. $)$ either, but showed a decrease in the stimulus-poor environment as compared to the stimulus-rich environment $(F(1,60)=5.34 ; p<0.05)$.

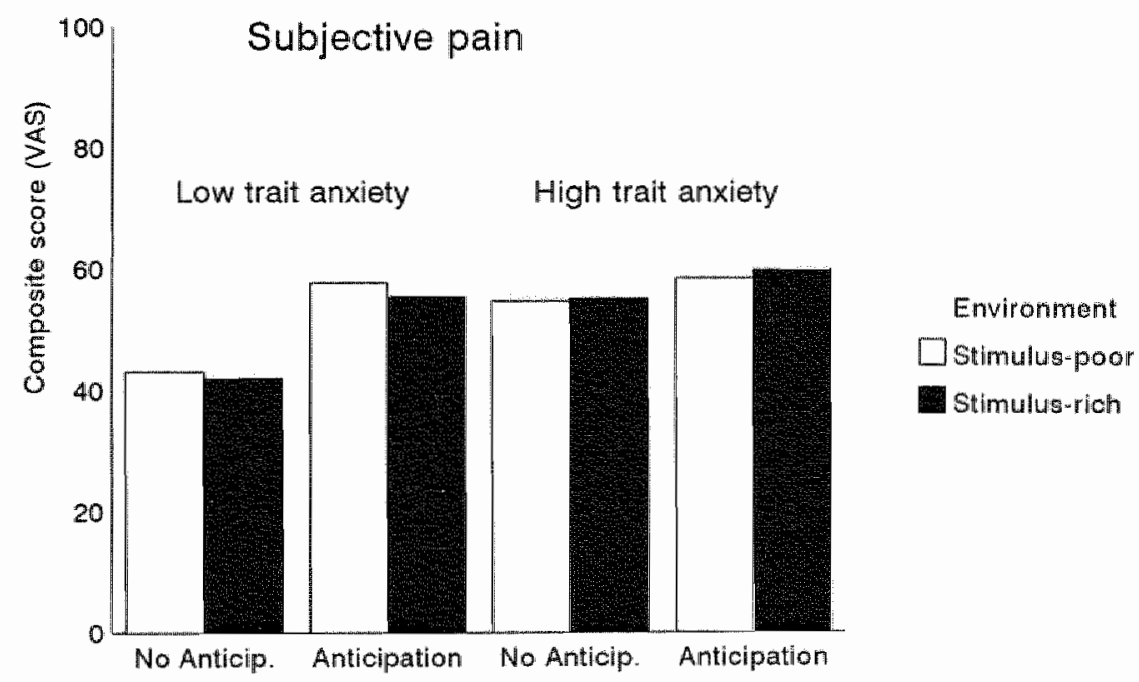

Figure 1. Mean subjective pain composite score for subjects low and high in trait anxiefy, with or without anticipatory anxiety, displayed for both stimulus-poor and stimulus-rich environment. 


\section{Discussion}

Since anxiety ratings of high trait socially anxious subjects were not significantly influenced by the anticipation of an oral presentation, the manipulation of anticipatory anxiety seems to have failed. Instead, high trait socially anxious subjects reported higher anxiety in the experimental situation than low trait socially anxious subjects, irrespective of anxiety manipulation. Although there was a significant effect of anxiety condition on skin conductance level, paradoxically only low trait anxious subjects showed heightened levels in response to the anticipation of an oral presentation. Interestingly, pain ratings followed the same pattern: there was an effect of anxiety condition on pain ratings, but especially low trait socially anxious subjects showed increased pain ratings in the anticipation as compared to the no anticipation condition.

In line with the original hypothesis, ratings of attention to pain were lower in the stimulus-rich than in the stimulus-poor environment, except for those of high trait socially anxious subjects anticipating an oral presentation, which appeared unaffected by environment. Thus, these high trait socially anxious subjects seemed to have automatically directed their attention towards the pain in both environments. This idea is strenghtened by the fact that high trait socially anxious subjects had less memory for neutral stimuli in the room, and slightly higher ratings of pain and attention to pain than low trait anxious subjects. However, environment did not significantly influence pain ratings in any of the experimental groups. Therefore it is unlikely that the observed effects of trait anxiety on pain were due to less distraction by the neutral stimuli. Nevertheless, it is possible that the low trait sccially anxious subjects, especially those in the no anticipation condition, actively tried to cognitively distract from pain, irrespective of environment. This strategy may have been impaired by the heightened anxiety levels in the high trait socially anxious subjects, and perhaps also in the low trait socially anxious subjects anticipating an oral presentation.

Given that they were socially anxious, why did high trait socially anxious

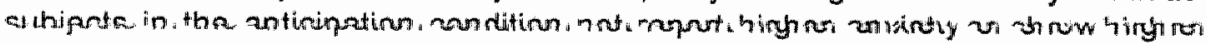
autonomic arousal than those in the no anticipation condition? They did report to spend more time thinking about the impending oral presentation than low trait socially anxious subjects in the anticipation condition, suggesting that they were more worried about it. Perhaps high trait socially anxious subjects in the no anticipation condition, after seeing the video tape, guessed that they were supposed to give a presentation and therefore became anxious. However, these subjects had very low ratings when after pain stimulation they were asked to what extent they had expected having to give an oral presentation, and all acted surprised when told. Another possibility is that subjects rated anxiety induced by the anticipation of pain rather than that induced by the anticipation of the oral presentation, either because they thought they were supposed to rate painrelated anxiety, or because they 'forgot' about the oral presentation during the pain stimulation trials. This does not explain, however, why anxiety ratings of high trait socially anxious subjects were higher than those of low trait socially anxious subjects. The high trait socially anxious subjects may have been generally more anxious in the (social) experimental situation than the low trait socially anxious 
subjects. Perhaps because of lower initial levels of autonomic arousal, only low trait socially anxious subjects showed heightened skin conductance levels in response to anticipation of an oral presentation "resulting in levels comparable to those of the high trait socially anxious subjects.

Despite the successful manipulation of attention as judged by attention ratings, environment did not have a significant effect on pain ratings. This is surprising, given the strong effects of aftention on pain in previous studles (Arntz, Dreessen \& Merckelbach, 1991; Arntz \& de Jong, 1993; Janssen \& Arntz, 1996). However, in those studies effects of attention manipulation on attention ratings were much larger, suggesting that in the present study the manipulation of attention by environment was just not strong enough to significantly affect subjective pain. On the other hand, a too strong manipulation of attention might result in distraction of attention from pain in all subjects, without being able to distinguish between those automatically directing attention towards pain and those more inclined to seek distraction. Another problem may have been that the pain stimulus had the same objective strength for all subjects, making the subjective level too strong for some to distract from. This might be resolved by individually calibrating the stimulus at a moderate level. Future studies in which pain-irrelevant anticipatory anxiety and attention are manipulated more successfully should determine whether differences in spontaneous attentional allocation play a role in the influence of anxiety on pain. 


$$
\text { . }
$$




\section{General discussion}

This final chapter recapitulates the results of the experimental studies. An attempt is made to integrate the various mechanisms that were studied into one model on the influence of anxiety on pain. Also, suggestions for future research and practical implications are discussed.

\subsection{Answering the research questions}

In six consecutive experimental studies the contributions of three possible mediators of the influence of anxiety on pain was investigated: attentional, endorphinergic, and adrenergic mechanisms. Four questions were addressed: 1) What is the role of endogenous opioids and attentional focus? 2) Do endogenous opioids inhibit pain during uncontrollable confrontation to anxiety-evoking stimuli in humans? 3) Does epinephrine in natural doses exert hyperalgesic effects? 4) Does anxiety lead to less distraction from pain due to a disturbance in attention regulation? As far as possible, an answer to each of these questions will be provided below on the basis of the experimental results.

\section{1) Endogenous opioids and attentional focus}

The experiments discussed in section 3.2 and 3.3 investigated the relative contribution of endogenous opioids and attentional focus in pain perception during phobic anxiety. This was done by administering an opioid antagonist in a paradigm in which anxiety and attention were independently manipulated. No evidence for a pain inhibitory effect of endogenous opioids was found in spider phobics being exposed to a spider in the laboratory. While a small pain-inhibiting effect of anxiety was observed in section 3.2, this could be explained by differences in aftentional focus. Furthermore, distraction from pain resulted in significantly lower paim ratings than attention focused on pain, irrespective of anxiety condition. Since effects of attentional focus were not influenced by the administration of naloxone, there is no evidence that endogenous opioids play a role in these pain-inhibiting effects of distraction. Although the pain-increasing effect of low dose naltrexone compared to placebo in section 3.2 suggests that pain in the placebo condition was inhibited by endogenous opioids, these effects were not specific to the anxiety conditions. While with respect to this first study it could be argued that, because of the mixed presentation of conditions, endogenous opioids released during anxiety may have continued to exert paininhibiting effects during the neutral conditions, this possibility is refuted by the study described in section 3.3, in which anxiety and neutral conditions were planned on separate days. No effect of anxiety on pain was found, and naloxone did not reverse any anxiety-induced pain inhibition. Rather, naloxone itself showed a dose-dependent pain inhibition, consistent with evidence that naloxone may have agonistic effects in the absence of endogenous opioid activity (Buchsbaum, Davis \& Bunney, 1977; Levine, Gordon \& Fields, 1979; Stacher et 
al. 1988). This interpretation is consistent with the absence of increased $\beta$ endorphim plasma levels during the anxiety session.

While there was a strong effect on pain of attention versus distraction in section 3.2 , the effect of attention condition did not reach significance in section 3.3. This may have been caused by the suddenness of the pain stimulus, which may automatically have led to the allocation of attention to pain. In an unpublished study (see section 3.3 , discussion), the effect of attention on pain was shown to depend on how fast the pain stimulus reached its maximum, casting doubt on the stability of the effect of attentional focus. However, the lack of an effect may also have been due to the failure to adequately manipulate attention on pain (as indicated by attention ratings), especially during the anxiety session. Several studies in which attention was successfully manipulated (section $3.2 \& 3.6$; Arntz, Dreessen \& Merckelbach, 1991; Arntz \& de Jong 1993; Arntz, Dreessein \& de Jong, 1994), demonstrated significant effects of attentional focus on pain. Overall, the results suggest that at least in the situation of spider phobic fear, effects of anxiety can be explained by attentional focus, and endogenous opioid mechanisms do not play a role.

\section{2) Endogenous opioids and uncontrollable anxiety}

Despite high levels of subjective anxiety, no evidence was found for endogenousopioid mediatled effects induced by exposure to a spider. From these results it was deduced that anxiety in humans does not necessarily result in endogenous opioid activity. A parallel was drawn between these human studies and animal research, where uncontrollability of the stressful situation was found to be a prerequisite for endogenous opioid effects (Maier et al., 1983). Perhaps, it was hypothesized, the situation of a spider exposure session in which subjects can set the pace of approach themselves, although inducing strong anxiety, was too controllable for the subjects to trigger the release of endogenous opioids. Therefore, the experiment described in section 3.4 comprised a more "flooding"like confrontation, during which a large spider was held immediately in front of spider phobic subjects for $5 \mathrm{~s}$. Again, however, no effect of an opioid antagonist was found in this anxiety-inducing situation, indicating that pain was not inhibited by endogenous opioids. Instead, reducing anxiety by administering the benzodiazepine alprazolam resulted in a slight pain inhibition, suggesting that anxiety was associated with a slight pain increase. For reasons discussed in section 3.4 , the anxiety affected by alprazolam was assumed to be pain-related. Subjective pain was argued to be decreased by alprazolam because the reduction in pain-related anxiety resulted in less attentional allocation towards the pain stimulus, or because it was associated with a reduction in sympathetic activation. This may have attenuated pain directly because adrenergic sensitization of nociceptors was inhibited, or indirectly because there was less arousal to be attributed to the pain.

Since even during a "flooding"-like confrontation with a phobic object no andogenous opioid effects were found, it was hypothesized that anxiety induced in the human laboratory may not be comparable to that induced in animal studies with regard to uncontrollability. Therefore, it was argued that research on stressinduced analgesia in humans should involve a situation which mimics 
uncontrollable real danger in an ethically acceptable way. Such a situation was found in a parachute jump, which had been used before by several researchers to experimentally induce a state of real-life stress outside the laboratory. Indeed, after a first-time parachute jump, endogenous opioid activity proved to have been triggered (section 3.5). $\beta$-endorphin plasma levels showed a threefold increase with respect to prejump levels, and subjects receiving placebo showed a decrease in subjective pain ratings and an increase in pain threshold compared to subjects in which opioid activity was blocked by naloxone. It was puzzling, however, that while this effect of naloxone suggests an analgesic effect of endogenous opioids released during the jump, the placebo group did not show a pain inhibition with respect to ratings immediately prior to the jump. Pain appeared to show a gradual inhibition from pretest measurement on, and returned to pretest pain levels by the administration of naloxone. This could be explained in terms of an opioid-mediated effect due to anticipatory anxiety, had not the rise in $\beta$-endorphin plasma levels strongly suggested that opioid activity was triggered during the jump and not before. As an alternative interpretation, it was speculated that next to an inhibition due to endogenous opioids in the placebo group, a second process may have increased pain in both the placebo and the naloxone group. Since pain sensitivity and perceived control during the jump proved to be negatively associated in a regression analysis, this second process was hypothesized to be related to loss of control during the jump. While on the one hand uncontrollability is believed to trigger endogenous opioid activity, on the other hand there is evidence that lack of perceived control increases pain sensitivity with respect to perceived contral (Arntz \& Schmidt, 1989). Although catecholamine release during the jump was proposed as a possible mediator of such a pain increase, the experimental design did not allow for examining a causal relationship between (nor)epinephrine release and pain sensitivity.

\section{3) Epinephrine-induced hyperalgesia}

In section 3.6, epinephrine and placebo were infused systemically to investigate epinephrine effects on pain. Increasing doses were infused in students low in fear of bodily sensations, the highest dose being comparable to epinephrine levels observed during extreme anxiety. Epinephrine induced a minor hyperalgesia with regard to electrical stimulation, but had no effect on pressure pain threshold. Attentional focus, although only varied during electrical stimulation trials, appeared to have much more impact on pain than epinephrine infusion. The finding of relatively small effects of epinephrine on electrical pain stimulation (compared to effects of attentional manipulation) corresponds to the absence of effects of anxiety in previous research (section 3.2 and 3.3) when attentional focus was controlled for. However, epinephrine exerted a significant hyperalgesic effect on heat pain threshold, which was even more pronounced when skin was sensitized with capsaicin. While similar effects had been found before in studies in which unnaturally high doses of (nor)epinephrine were peripherally infused into the skin, we found pain-increasing effects of systemic epinephrine in natural doses, which is directly relevant to the question of whether anxiety influences the perception of pain. However, it is not very likely that epinephrine mediates any profound increases in pain experience during anxiety, since its effects on 
subjective pain due to electrical stimulation were only very small and effects were even absent on pressure pain threshold. Even the statistically significant effect on heat pain threshold was very small, and would only explain very subtle effects of anxiety an pain. Nevertheless, the effect on heat pain threshold in capsaicintreated skin suggests that epinephrine may importantly affect pain perception when skin is sensitized or injured.

\section{4) Anticipatory anxiety and distraction from pain}

The experiment in section 3.7 investigated whether pain-irrelevant anticipatory anxiety is associated with less spontaneous distraction from pain than no anticipatory anxiety. Although the manipulation of anticipatory anxiety for an oral presentation within high trait socially anxious subjects did not prove successful, some indications were found for a disturbance in attentional regulation when looking at differences between high trait socially anxious and low trait socially anxious subjects. High trait socially anxious subjects, who generally reported more anxiety in the experimental situation than low trait socially anxious subjects, reported slightly stronger pain, more attention towards pain and less memory for neutral objects in the environment. This is consistent with the idea that the high trait anxious group may not, or to a lesser degree than the low trait anxious group, distract themselves from pain by directing their attention towards neutral stimuli. However "trait and state factors are not easily separated: high socially anxious subjects may generally attend less to their environment and more to pain than low socially anxious subjects, whether they are in an anxious state or not. Vice versa, it is also possible that high socially anxious subjects are generally more sensitive to pain stimulation, which may in turn have resulted in higher anxiety ratings and more attention towards pain. Manipulating anxiety and attention, therefore, still seems a better way to investigate influences of anxiety on pain.

\section{Concordance between subjective and psychophysiological measures}

The results recapitulated above mainly referred to subjective pain ratings and pain thresholds. However, in most studies skin conductance response and heart rate response to electrical stimulation were also measured. Sometimes the effects of anxiety or attentional focus on psychophysiological responses were consistent with the effect on subjective pain, but just about as often they were not. In section 3.2 , skin conductance responses showed a quadratic effect of naltrexone and no effect of anxiety, similar to the effect on subjective pain. However, while subjective pain was markedly decreased by distraction skin conductance responses showed an increase in the distraction versus the attention conditions. Furthermore, the inhibiting effect of naloxone on subjective pain in section 3.3 , and the marginally significant effect of alprazolam on subjective pain in section 3.4 were not accompanied by similar effects of naloxone and alprazolam on heart rate responses to pain. In section 3.6, an effect of attention versus distraction was found both on subjective pain and on heart rate response, but not on skin conductance response. Although there was a trend towards an increasing effect, epinephrine did not significantly influence subjective pain ratings and heart rate response. In contrast, skin conductance response showed an inhibition with 
epinephrine as compared to placebo infusion. In section 3.7 , the effects on psychophysiological measures were reminiscent of those in the epinephrine study: skin conductance responses were slightly lower among high trait socially anxious subjects, who reported situational anxiety, compared to low trait socilally anxious subjects, while heart rate response showed an effect of attention manipulation, responses being lower in the stimulus-rich than in the stimulus-poor environment.

Overall, skin conductance response appears to be related both to the unpredictability and to the experienced intensity of pain, which explains the dissociated effects on SCR and subjective pain. Heart rate response appears, at least in the studies reported here, to correspond more than SCR with experienced pain intensity, and is affected by attentional focus in those studies in which subjective pain is. However, in two studies (section 3.4 and 3.7) a heart rate deceleration was observed in response to painful stimulation, whereas the defensive reaction to pain is belleved to involve a heart rate acceleration (Turpin, 1986). Since these were the only two studies in which no calibration procedure was used before the experimental trials, it may be argued that 1) the pain stimulation was too weak to induce a defensive reaction, or 2) pain-related anticipatory anxiety increased the mean heart rate just before pain stimulation, masking responses to the pain.

\subsection{Towards an integrated model}

The experiments discussed in the present thesis have provided evidence for an influence on pain of attentional focus, endogenous opioids, as well as epinephrine release during anxiety. These various mechanisms appeared to have independent effects, since no interactions were found between effects on pain of naltrexone/naloxone and attentional focus (section 3.2 and 3.3 ), epinephrine and attentionall focus (section 3.6), and naloxone and epinephrine release (section 3.5). If all of these mechanisms play a role in the influence of anxiely on pain, what can be said about the influence anxiely will have in a particular situation? Does anxiety inhibit pain by the release of endogenous opioids, does it increase pain by the release of epinephrine, or does the effect depend on the way in which anxiety determines the focus of attention? Since the evidence for attentional, endorphinergic and adrenergic mechanisms came from very different fields of study, so far no model of anxiety and pain exists that incorporates all of these mechanisms. Studies investigating effects of anxiety on pain usually addressed only a single mechanism, whereas the experienced pain in any situation may be a resultant of all of them. Even in the present thesis, evidence for different mechanisms was often found in separate experiments, and it is not entirely clear which mechanism under what circumstances may be expected to have a dominating effect on pain. Nevertheless, the results of the experimental studies in this thesis do provide some clues with respect to the question when each mechanism exerts an influence. An attempt will be made below to integrate the various mechanisms that were studied into a preliminary model on the influence of anxiety on pain. 


\section{Endogenous oplorids}

Whereas high levels of phobic anxiety did not induce endogenous opioid activity, even lower anxiety levels were accompanied by an opioid-mediated analgesia in novice parachute jumpers. It seems that, for triggering endogenous opioid effects during anxiety, a certain amount of uncontrollability or helplessness is required. This is consistent with indications that threatening situations differing in perceived control are associated with different response patterns and neuroendocrine responses (Henry, 1990). In a threatening situation that is perceived as potentially controllable or escapable, subjects will actively seek ways to cope with it, either by fighting it or by fleeing from it. When an animal feels it can cope with a challenge, it becomes angry and fights. Norepinephrine release is then the typical neuroendocrine response. When, instead of anger, fear is dominant, epinephrine rises to enable several adaptations necessary for the explosive expression of flight behaviour. Henry discriminated another state of anxiety, however, in which submissive behaviour and feelings of helplessness are dominant. In this case, the adrenal cortex is actiwated and ACTH (adrenocorticotropic hormone) is released, which is a first link in the sequence of ACTH-corticosterone-endorphin release. Interestingly, it has been suggested that endogenous opioids may be released to pose a limit to extreme sympathetic activation and its consequences (Grossman, 1988). Indeed, Bouloux ef al. (1985) found that with extreme sympatheticactivation naloxone hascatecholamine-increasing effects, suggesting that endorphins inhibit catecholamine release.

The degree of control that is perceived with regard to a particular stressor appears to determine whether that stressor elicits agression, escape, or submissive behaviour. Simplifying, these behaviaurs and their associated neuroendocrine responses might be triggered respectively by what is perceived as a challenge to control, a threat to control, or a loss of control. Loss of perceived control seems to be a prerequisite for endogenous opioid activity to be triggered; either subjects must have learned that they have no control over the threatening stimulus, or the threat must be so overwhelming that subjects do not even try to obtain control. Rats are known to show anaigesic effects, submissive behaviour and freezing when they are in the presence of a dominating enemy or a natural predator. While the pain reactivity of animals that can control pain offset is unaffected by naloxone, yoked animals experiencing the same amounts of shock without being able to control it show an analgesila that is reversed by naloxone (Maier et al., 1983). Endogenous oploid-mediated analgesic effects in rats were observed only when a stressor was provided long enough to enable the rats to learn they had no control over it (Watkins \& Mayer, 1982). In humans, analgesic effects reversed by naloxone were demonstrated when subjects were anticipating unpredictable high intensity stimulation (Willer \& Ernst, 1986), when traumatized Vietnam veterans were shown a combat video (Pitman et al., 1990), or when subjects were exposed to an extremely threatening situation outside the laboratory, a first-time parachute jump (section 3.5). It is interesting that in Pitman et al. (1990) the veterans, next to reporting more fear and anger than healthy control subjects, also reported more sadness and less control. It is highly plausible that in the past, the traumatized Vietnam veterans had experienced a severe loss of control in situations similar to those shown in the combat video. 
The question could be raised in which way the inhibition of pain by endogenous opioids, observed when active coping with a threat is (no longer) possible, may contribute to the survival of the organism. Bolles \& Fanselow (1980) assumed that anxiety has priority over pain in order to promote active coping with the threat. They argued that, through the release of endogenous opioids, pain is inhibited during anxiety to prevent it from interfering with fight or flight behaviour. However, this idea is inconsistent with the evidence that endogenous opioids are released only during situations perceived as uncontrollable or inescapable. In such situations, fight or flight behaviour may not even be initiated. Besides their uncontrollable nature, the situations in which an opioid-mediated analgesia was found have in common that anxiety was induced by the threat of physical harm. This suggests that endogenous opioids help to diminish the impact of actual or imminent physical harm when other ways of coping have failed. Submissive behaviour and freezing may be viewed as adaptive in such situations, since they might temper the aggressive behaviour of an opponent, will avoid the attraction of attention during hiding, and may even suggest death. The adaptive value of analgesic effects may therefore be that they facilitate freezing behaviour and inhibit responses to the pain that may attract the attention of an opponent. Apart from its survival value, an endogenous opioidmediated analgesia may have beneficial side-effects, as illustrated by the following dramatic anecdote from David Livingstone, the well-known missionary and explorer of Africa,

.... I heard a shout. Starting, and looking half found, I saw the lion just in the act of springing upon me. I was upon a little height; he caught my shoulder as he sprang, and we both came to the ground below together. Growing horribly close to my ear, he shook me as a terrier does a rat. The shock produced a stupor similar to that which seems to be felt by a mouse after the first shake of the cat. It caused a sort of dreaminess in which there was no sense of pain nor feeling of terror, though quite conscious of all that was happening. It was like what patients partially under the infuence of chloroform describe, who see all the operation, but feel not the knife.... The shake annihilated fear. and allowed no sense of horror in looking round at the beast. This peculiar state is probably produced in all animals killed by the carnivora; and if so, is a merciful provision by our benevolent creator for lessening the pain of death.

(Livingstone, Missionary Travels, 1857)

\section{Epinephrine}

No endagenous opioid effects were observed during anxiety induced by exposing spider phobic subjects to a spider. It is conceivable that the exposure to the phobic object was not accompanied by feelings of helplessness, but was perceived as potentially controllable. Consequently, this anxiety-provoking situation may have elicited the inclination to escape, and epinephrine may have been the primary neuroendocrime response. Thus, although confrontation with the spider did not result in the release of endogenous opioids, it may have been accompanied by epinephrine release. Since systemic epinephrine was shown to produce hyperalgesic effects (section 3.6), one would expect pain experience to be increased during confrontation with a potentially controllable threat. Although in section 3.3 , in which endocrine levels were determined, no indications were found for an increase in epinephrine plasma level during phobic anxiety, this could be attributed to the fact that blood was taken after each trial, missing 
possible effects of anxiety on epinephrine because of its fast metabolism. No pain-increasing effects of phobic anxiety were found in section 3.2 and 3.3 , but the effect of the benzodiazepine alprazolam in section 3.4 , reducing both anxiety and pain, may be viewed as evidence for a pain increase during anxiety. However, like the effect of epinephrine infusion on the experience of electrical pain stimulation, the effect of alprazolam was only a trend, and too small to reach significance. It is therefore unlikely that the increases in pain which are often reported in human studies on the influence of anxiety on pain (see Chapter 2 ) can be entirely explained by the anxiety-related release of epinephrine.

Nevertheless, it seems that epinephrine may affect the pain experienced during tissue sensitization or injury. In sensitized skin the effects of epinephrine were significantly larger, at least on heat pain threshold. Particularly when tissue is damaged, hyperalgesic effects of epinephrine released during anxiety may serve to increase the warning function of pain. In situations of injury it may be critical that the injured site is protected and healing is promoted. On the other hand, as also noted by Bolles \& Fanselow (1980), it may be just as critical that pain does not interfere with fight or flight behaviour. Although they proposed endogenous opioid release as a mechanism, it may be argued that attentional mechanisms are a more likely candidate. Assuming that attention will only be directed to those aspects of the environment that are crucial to escape, attention will be distracted from pain during situations in which flight is initiated. Effects of attentional focus, although only measured on normal skin, turned out to be much larger than the epinephrine-induced hyperalgesia. Thus, when necessary, effects of attentional focus may easily overrule epinephrine-induced hyperalgesic effects and lead to a pain inhibition to facilitate fight or flight activity.

\section{Attentional focus}

The results reported in this thesis confirm the already strong evidence that attentional focus plays an important role in the influence of anxiety on pain. The presence of an object of fear that was irrelevant to the pain (the spider) did not exert an influence on pain when the resultant distraction from pain was controlled for (section 3.2). This indicates that pain-irrelevant anxiety inhibits pain by leading to the allocation of attention towards the abject of fear, away from pain. Only when the object of fear is absent (as during anticipatory anxiety), pain-irrelevant anxiety may be expected to be accompanied by an increase in pain. It was argued that during anxiety, attention regulation strategies may be disturbed and attention may automatically be focused on the most threatening aspects of the situation, usually including the pain. Some weak indications were found for the hypothesis that high anxiety is associated with less spontaneous distraction from pain (section 3.7 )

In the case of anxiety related to pain or injury, attention will rather be focused on the pain than be distracted from pain, resulting in heightened sensitivity for pain. This is not necessarily a disadvantage, since the experience of pain may indicate the occurrence of tissue damage and thus act as a warning signal to prevent further injury. However, when fear is exaggerated or when the paininducing situation is beyond the subject's control, this attentional focus on pain becomes maladaptive. In this case, the allocation of attention towards pain and 
the release of epinephrine will be expected to concomitantly lead to a pain increase. Although only a speculation, the prediction could be madie that when this situation is prolonged and induces a state of learned helplessness, this pain increase may be partially counteracted by the release of endogenous opioids.

\subsection{Suggestions for future research}

Some of the research questions were partially left unanswered, the answer to others raised new research questions. Some suggestions may be made for future research and for how to solve some of the problems encountered in the present thesis.

Since the manipulation of anxiety and attentional focus in the last study (section 3.7) was not successiul, the last research question, whether anxiety leads to less distraction from pain, could not be satisfactorily answered. Perhaps the anxiety manipulation would have been more successful if the pain trials and the feared situation were less separated, or if it had been stressed to subjects that general anxiety level and not only anxiety about pain, should be reported. Another problem may have been that the pain was too strong, making it hard to distract from for all subjects, without discriminating between anxious and nonanxious subjects. This may be solved by calibrating the pain stimulus at a moderate perceived intensity level prior to the experimental trials. Also, the attention manipulation may not have been strong enough to distract from pain, given that even low trait anxious subjects did not show effects of a distractive environment on pain. However, caution should also be taken to not make attention manipulation so strong that all subjects will be distracted, since the aim was to investigate differences in spontaneous distraction.

With regard to the experiment with parachute jumpers (section 3.5 ) some questions were left unanswered as well. Most importantly, it is not clear exactly at which point in the jump sequence endogenous opioid activity was triggered. The pain data suggest that an opioid-mediated analgesia already sets in before the jump, since pain levels return to pretest level with the administration of naloxone after the jump. Nevertheless, the observed threefold increase in $\beta$ endorphin plasma levels after the jump and the absence of a rise before the jump strongly suggests that endogenous opioids were triggered during the jump and not before. The relationship between peripheral and central opioids is far from perfect, however, and central opioid activity may have preceded $\beta$-endorphin release. Unfortunately, for both practical and ethical reasons it is impossible to measure central or cerebrospinal opioid levels during an experiment. Although this may also cause ethical problems, ideally naloxone or placebo should be given during the entire jump sequence, with booster doses before each pain measurement. In this way, it could be tested at which moment opioid activity starts to influence pain sensitivity. However, if a pain increase in the naloxone with respect to the placebo group would be observed prior to the jump, it would still be unclear whether anticipatory anxiety or a habituation process was responsible for endogenous opioid activity. To rule out the possibility that the observed pain inhibition before the jump was due to habituation processes, it would be advisable to include in the design a control group that is not jumping. 
but in which the same measures are taken. If a naloxone-reversable habituation cannot be observed in this group, a prejump decrease in pain sensitivity observed in subjects making a jump must be due to anticipatory anxiety prior to the jump.

While the pathologically high levels of anxiety shown by spider phobic subjects in the presence of a spider did not induce a naloxone-reversable analgesia, similar or even lower subjective levels of anxiety were accompanied by opioid activity in novice parachute jumpers. This is consistent with the hypothesis that next to anxiety, a certain amount of uncontrollability is required to trigger endogenous opioid activity. Thus, the degree of perceived control seems to be an important factor determining the influence of anxiety on pain. However, since perceived control was not varied within any of the experimental studies reported here, the only direct evidence for this was derived from animal studies (Maier et al., 1983). In fact, in studies with humans in which perceived control was experimentally manipulated, perceived control was often associated with higher pain thresholds than the absence of control (for a review see Arntz \& Schmidt, 1989). Thus, when subjects are provided with a means to control several aspects of the pain, pain is tolerated longer than when the pain is perceived as beyond their control. This leads to a paradox: in what way is this effect of perceived control related to the inhibitory effects during uncontrollability? According to Bandura (1987), a nonopioid mechanism would subserve pain tolerance while cognitive control is effectively exercised, but an opioid mechanism would come into play in later stages of coping when control techniques are no longer sufficient to attenuate increasing pain or to block it from consciousness. Only when the threat is prolonged, a state of uncontrollability or helplessness will be experienced and endogenous opioid effects will be expected. Before that stage, however, low perceived control would only have detrimental effects.

It would be interesting to test experimentally whether failing control (the subject tries to obtain control but fails) leads to higher pain sensitivity than perceived control in a pain situation, while a situation in which the absence of control is experienced (providing no possibility to obtain control at all) will lead to intermediate pain sensitivity, since both nonopioid pain-increasing effects and opioid pain-inhibiting effects will be expected to influence pain. Perceived control could be manipulated by asking subjects to find a code, which works to stop the pain after a few trials (perceived control), is never found (failing control), or is told not to have any relation to the pain (absence of control). The possible mediating effects of endogenous opioids could be investigated by means of naloxone. The mechanisms involved in pain-reducing effects of perceived control are unclear, although the perception of control has been argued to attenuate aversive physiological arousal, bodily tension and epinephrine release, or to allow one to distract attention from pain (Bandura, 1987; Arntz \& Schmidt, 1989).

The studies reported in this thesis aimed to investigate general effects of anxiety on pain. However, next to the type of anxiety, the type of stimulus seemed to be critical as well. Effects of epinephrine on pain were less clear with electrical pain stimulation than with heat pain threshold, the effects on the latter in its turn being smaller on normal skin than on sensitized skin. In the epinephrine study effects were demonstrated with different methods of stimulation, that were not directly comparable. Although it was concluded that effects of attentional 
focus were much larger than effects of epinephrine, this cannot be generalized to other pain measures, since it was only assessed with electrical stimulation. Likewise, the effect on sensitized skin was only assessed within heat pain stimulation. It would be interesting to study effects of anxiety on pain experience due to spontaneous pain from injury or due to pain stimulation on sensitized skin.

\subsection{Implications for clinical practice}

Which are the implications of the experimental findings for the experience of pain during medical or dental treatment or for the understanding of chronic pain states? Although the studies reported were primarily concerned with fundamental questions, several links can be made.

The primary reason for pain-increasing effects of anxiety observed in many clinical pain situations may be that, if in those situations anxiety is present, it will usually be pain-related. Pain-related anxiety may result in an attentionall allocation towards the pain, and in this way exacerbate the pain experience. Therefore, the most obvious strategy to reduce the pain would be distraction from the pain. Although epinephrine release may exert a minor pain-increasing effect, the focus of attention seems capable of overruling physiological effects. However, when the pain is the result of an injury, epinephrine effects may be more pronounced. Also, the experience of pain may sometimes be too strong to distract attention from, or pain-related anxiety may automatically result in attention towards pain, whether a distractive environment is provided or not. In such cases, it may be fruitful to seek ways to reduce anxiety for pain. Benzodiazepines may be of some help here, considering indications that they reduce the unpleasantness of pain by reducing situational anxiety. Since autonomic responses were found to be sensitive to the unexpectedness of pain, it may be wise to strive for predictable pain by announcing every painfull infliction.

Of course, being an aversive experience in itself, anxiety should be prevented when possible. Or should it? In the present thesis, several indications were found that anxiely may inhibit pain, either by distracting attention from pain, or by triggering the release of endogenous opioids. Would it be advisable lo scare people receiving dental treatment by the presence of a nuge spider? Probably not, since they could just as well be distracted by an entertaining fragment of L.A. Law. Could then be benefitted from the wholesome analgesic effect of endogenous opioids, by lowering subjects" perceived control in a painful situation? It is obvious that this would only increase suffering, if not because of the pain than because of anxiety. Moreover, in acute pain situations the absence of control only seems to have pain-increasing effects as compared to situations in which control is perceived (Arntz \& Schmidt, 1989). However, prolonged pain or threat that is perceived as beyond control may trigger the release of endogenous opioid activity. In such situations, reducing arnxiety, for instance by the administration of benzodiazepine, may increase the pain experience (e.g. Willer \& Ernst, 1986).

One might wonder whether this also holds true for chronic paim patients. Since these patients suffer from long-lasting pain that they usually perceive as beyond their control, they may be expected to show enhanced endogenous opioid 
activity. Instead, chronic pain patients have been found to show relatively low $\beta$ endorphin levels in cerebrospinal fluid (Chapman \& Bonica, 1983). It is possible that a prolonged state of learned helplessness may lead to a tolerance for and eventually a fall in endogenous opioid peptides. Other mechanisms investigated in this thesis, in particular the focus of attertion, may also affect the experience of chronic pain. Especially in the case of anxiety for pain or further injury. attention may be focused on the pain, usually at the cost of activities that would potentially distract attention from the pain. Although this may temporarily be reversed by distraction techniques, the beneficial effects of distraction may be more directly applicable to acute pain situations. In chronic pain patients, attention will be focused on pain again if anxiety about the pain persists. Also, anxiety about the pain may increase epinephrine release and muscle tension, leading to a further increase in pain. Therefore, anxious chronic pain patients may benefit more from interventions that are almed at reducing anxiety than from distraction. 


\section{References}

Ahles, T.A., Blanchard, E.B. \& Leventhal, H. (1983). Cognitive control of pain. attention to the sensony aspects of the cold pressor. Cognitive Therapy and Research, 7(2), 159-178.

Ahles, T.A., Cassens, H.L. \& Stalling, R.B. (1987). Private body consciousness, anxiety and the perception of pain. Joumal of Behavior Therapy and Experimental Psychiatry, 18 $(3), 215-222$.

Akil, H., Watson, S.J., Young, E. Lewis, M.E., Khachaturian, H. \& Walker, J.M. (1984), Endagenous opioids: biology and function. Annual Review Neuroscience, 7, 223-255.

Al-Absi, M., Buchanan, T. \& Lovallo, W.R. (1996). Pain perception and cardiovascular responses in men with positive parental history for hypertension. Psychophysiology, 33, 655-661.

Al-Absi, M. \& Rokke, P.D. (1991). Can anxiety help us tolerate pain? Pain. 46, 43-51.

Allport, A. (1993). Attention and control: have we been asking the wrong questions? A critical review of twenty-five years. In: D.E. Meyer \& S. Kornblum (Eds.), Aittention \&erformance (XIV, pp. 183218), MIT Press, London.

Andriessen, J.H.T.H. van Cadsand, J.P. (1983) Een analyse van de Nederlandse I.E.-schaal (An analysis of the Dutch I.E scale). Nederlands Tijdschrift voor de Psychologie, 38, 7-24.

Arnsten, A.F.T., Segal, D.S., Neville, H.J., Hillyard, S.A., Janowski, D.S., Judld, L.L.\& Bloom, F.E. (1983). Naloxone augments electrophysiological signs of selective attention in man. Nature, 304 . 725-727.

Arntz, A. Dreessen, L. \& Merckelbach, H. (1991). Attention, not anxiety, influences pain. Behaviour Rosearch and Therapy, $29_{4} 41-50$.

Arntz, A., Dreessen, L. \& de Jong, P. (1994). The influence of anxiety on pain: attentional and attributional mediators. Pain, 56, 307-314.

Arntz, A., wan Eck, M \& Heijmans, M. (4990). Predictions of dental pain: the fear of any expected ewil is worse than the evill itself. Behaviour Research and Therapy, 28, 87-90.

Antz, A. \& de Jong, P. (1993) Anxiety, attention and pain. Journal of Psychosomatic Research, 37, $423-432$.

Arntz; A., Merckelbach, H. \& de Jong, P. (1993). Opioid antagonist affects behavioral effects of exposure in vivo. Jaurnal of Consulting and Crinical Psychology, 61(5), 865-870.

Arntz, A. Lavy, E., Berg, G. van den, \& Rijsoort, S. van (1993b). Negative beliefs of spider phobics: A psychometric evaluation of the Spider Phobia Beliefs Questionnaire. Advances in Behaviour Research and Therapy, 21, 1-12.

Arntz, A., Schmidt, A.J.M. (1989). Perceived control and the experience of pain. In A. Steptoe \& A. Appels (Eds.) Stress, Personal Control and Healih. John Willey and Sons. Chichester.

ASHP American Society of Hospital Phamacists (1989). American Hospital Formulary Service Drug information (pp. 1070-1076). Bethesda.

Bandura, A., OLeary, A., Barr Taylor, C., Gauthier, J. \& Gossard, D. (1987). Perceived self-efficacy and pain control: opioid and nonopioid mechanisms. Joumal of Personality and Social Psychology, 35 (3), 563-571.

Barasi $S$. Lynn, B. (1986). Effects of sympathetic stimulation on mechanoreceptive and nociceptive afferent units from the rabbit pinna. Brain Research, 378, 21-27.

Beecher, H.K. (1956). Relationship and significance of wound to the pain experienced. Joumal of the American Medical Association. 161, 1609.1613.

Bobey, M.J. \& Dawidson, P.O. (1970). Psychological factors affecting pain tolerance. Journal of Psychosomatic Research, 14, 371-376.

Bolles, R.C. \& Fansellow, M.S. (1980). A perceptual-defensive-recuperative model of fear and pain. The Behavioral and Brain Sciences, 3, 291-323.

Bouloux., P.M., Grossman. A., Al-Damluji, S., Bailley, T, Besser, M. (1985). Enhancement of the sympathoadrenall response to the cold-pressor test by naloxone in man. Clinical science, 69, 365368 .

Brewer, B.W. \& Karoly, P. (1989). Effects of attentional fucusing on pain perception. Motivation and Emotion, 13 (3), 193-203.

Broadbent "D.E. (1958). Perception and Communication, Pergamon Press, London. 
Buchsbaum, M.S. Davis, G.C. Bunney, W.E. (1977). Naloxone alters pain perception and somatosensory evoked potentials in normal subjects. Nature, $270,620-622$.

Caceres, C. Bums, J.W. (1997). Cardiovascular reactivity to psychological stress may enfrance subsequent pair sensitivity. Pain, 69, 237-244.

Carter, L.E. McNeill, D.W. \& Reed, T.L. (1991). Cardiac reactivity to combinations of anxiety and pain (abstract). Psychophysiology, 28, 516.

Chambless, D.L., Caputo, G.C., Bright, P. \& Gallagher, R. (1984). Assessment of fear of fear in agoraphobics: The Bodily Sensations Questionniaire and the Agoraphobics Cognitions Questionnaire. Journal of Consusting Clinical Psychology, 52. 1090-1097.

Chance, W.T., White, A.C., Krynock, G.M. \& Rosecrans, J.A. (1979). Autoanalgesia: Acquisition, blockade, and relationship to opiate binding. European Journal of Pharmacology, 58, 461-468.

Chapman, C.R. \& Bonica, J.J. (1983). Acute pain. Current Concepts, The Upjohn Company.

Chapman, C.R. Feather, B.W. (1973). Effects of diazepam on human pain tolerance and pain sensitivity. Psychosomatic Medicine, 35, 330-340.

Chapman, C.R. \& Tumer, J.A. (1986). Psychological control of acute pain. Joumal of Pain and Symptom Management, $1,9-20$.

Choi, B. Rowbotham, M.C. (1997). Effect of adrenergic receptor activation on post-herpetic neuralgia pain and sensory disturbances. Pain, 69, 55-63.

Christianson, S.A. (1992). Emotional stress and eyewitness memory: a critical review. Psychological Bulletin, 112, 284-309.

Cohen, M.R., Pickar, D. \& Dubois, M. (1983). The role of the endogenous opioid system in the humain stress response. Psychiatric Clinics of North America, 6(3), 457-471.

Cornwall, A. \& Donderi, D.C. (1988). The effect of expierimentally induced anxiety on the experience of pain. Pain, 35, 105-103.

Curtis, G.C. \& Glitz, D.A. (1988). Neuroendocrine findings in anxiety disorders. Endocinology of Neuropsychialric Disorders, 17(7), 131-147.

Dawson, M.E., Schell, A.M. \& Fillon, D.L. (1990), The electrodermal system. In J.T. Cacioppo \&. L.G. Tassinary (Eds.) Principles of Psychophysiology: Physical, social, and inferential elements. Cambridge University Press, Cambridge.

Dellemijn, P.L.I. \& Fields, H.L. (1994). Do benzodiazepines thave a role in chronic pain management? Pain. 57, 137-152.

Derogatis, L.R., Lipman, R.S. \& Covi, L. (1973), SCL-90: An outpatiënt psychiatric rating scalepreliminary report. Psychopharmacological Bulletin, 9, 3-2B.

Dimsdale, J. \& Moss, J. (1980). Short-term catecholamine response to psychological stress. Psychosomatic Medicine, 42 (5), 493-497.

Dingledine, R., lversen, L.L. \& Breuker, E. (1978). Naloxone as a GABA antagonist: evidence from iontophoretic, receptor binding and convulsant studies. European Joumal of Pharmacology, 47, $19-27$.

Dougher, M.J. (1979). Sensory decision theory analysis of the effects of anxiety and experimental instructions an pain. Journal of Abnormal Psychology, 88 (2), $137-144$.

Dougher, M.J., Goldstein, D. \& Leight, K.A. (1987). Induced anxiety and pain. Journal of Anxiety Disorders, $1.259-264$.

Drummond P.D. (1995). Noradrenaline increases hyperalgesia to heat in skin sensitized by capsaicin Pain, 60, 311-315.

Drummond, P.D. (1996). Independent effect of ischaemia and noradrenaline on thermal hyperalgesia in capsaicin-treated skin. Pain, 67, 129-133.

Easterbrook, J.A. (1959). The effect of emotion on cue utilization and the organization of behavior. Psychological Review, 66, 183-201.

Eccleston, C. (1995). The attentional control of pain: methodological and theoretical concerns. Pain, 63. 3-10.

Eysenck, M.W. (1988). Anxiety and attention. Anxiety Research, 1, 9-15.

Fanselow, M.S. Helmstetter, F.J. (1988). Conditional analgesia defensive freezing and benzodiazepines. Eehavioral Neuroscience, 102, 233-243.

Fanselow, M.S. (1986). Conditioned fear-induced opiate analgesia: a competing motivational state theory of stress analgesial. In D.D. Kelly (Ed.), Stress-induced Analgesia, Annals of the New York Academy of Sciences, 467 (pp. 40-54), New York.

Fanselown M.S. (1991). Analgesia as a response to aversive Pavlovian conditional stimuli: cognitive 
and ermotional mediators. In M.R. Denny (Ed), Fear, avoidance and phobias: a fundamental analysis. Erlbaum, N.Y.

Fenz, W.D., Epstein, S. (1967). Gradients of physiological arousal in parachutists as a function of an approaching jump. Psychosomatic Medicine, 29(1), 33-51.

Fenz, W.D. Jones, G.B. (1972). Individual differences in physiologic arousal and performance in spoit parachutists. Psychosomatic Medicine, 34(1), 1-8.

Fey, J.A., van Zuilen, R.W. (1984). Handleiding bij de spanningsbehoeftelijst (SBL) (Manual of the Dutch sensation seeking scale). Swets \& Zeittinger, Lisse.

Forgione, A., Barber, T. (1971). A strain gauge pain stimulator. Psychophysiology, 6, 102-106.

Gear, R.W. Miaskowski, C., Heller, P.H., Paul, S.M., Gordon, N.C. \& Levine, J.D. (1997) Benzodiazepine mediated antagonism of opioid analgesia. Pain, 74, 25-29.

Gross, R.T. \& Collins, F.L. (1981). On the relationship between anxiety and pain: methodological confounding. Clinical Psychology Review, 1, 375-386.

Grossman, A. (1988). Opioids and stress in man. Joumal of Endocrinology. $149,377-381$.

Groves, P.M. \& Thompson, R.F. (1970). Habituation: a dual-process theary. Psychological Review. $77,419-450$.

Hall, K.R.L. \& Stride, E. (1954). The varying response to pain in psychiatric disorders: at study in abnormal psychology. British Journal of Medical Psychology. 27, 48-60.

Hayes, R.L., Bennett, G.J., Newlon, P.G. \& Mayer, D.J. (1978). Behavioral and physiological studies of non-narcotic analgesia in the rat elicited by certain environmental stimuli. Brain Research. 155 . 69-90.

Haslam, D.R. (1966). The effect of threatened shock upon pain threshold. Psychonomic Science, 6 (6), 309-310.

Hennig, J., Laschefski, $U_{1,}$ Opper, $C$. (1994). Biopsychological changes after bungee jumping: $\beta$ endorphin immunoreactivity as a mediator of euphoria? Neuropsychobiology, 29, 28-32.

Henry, J.P. (1990). Stress, neuroendocrine patterns, and emotional response. In Noshpitz, J.D. \& Caddington, R.D. (Eds.), Stressors and the Adjustment Disorders (pp 477-496). New York: Wiley.

Hades, R.L., Howland, E.W. Lightfoot, N. \& Cleelland, C.S. (1990). The effects of distraction on responses to cold pressor pain. Pain, 41, 109-114.

Hoffman-Goetz, L., Pederson, B. (1995). Psychological aspects of stress immunology (Reply). Immunology Today, 16(3), 165.

van der Hoorn, F.A., Boomsma, F., Man in't Veld, Schalekamp, M.A.D.H. (1989). Determination of catecholamines in human plasma by high-performance liquid chromatography: comparison between a new method with fluorescence detection and an established method with electrochemical detection. Joumal of Chromatography, 487, 17-28.

Hu, S. \& Zhu, J. (1989), Sympathetic facilitation of sustained discharges of polymodal nociceptors. Pain, 38, 85-90.

James, W. (1890). Principles of Psychology. Holt, N.Y.

Janal, M.N., Colt, EW.D. Clark, W.C. G Glusman, M. (1984). Pain sensitivity, mood and plasmi endocrine levels in man following long-distance running: effects of naloxone. Pain, 19, 13-25.

Janssen, S.A. \& Arntz, A. (1996). Anxiety and pain: attentional and endorphinergic influences. Pain. $66,145-150$.

Janssen, S.A. \& Arntz, A. (1997). No ewidence for opioid-mediated analgesia induced by phobic fear. Behaviour Research and Therapy, 35 (9), 823-830.

Janssen, S.A., Arntz, A.\& Bouts, S. (1998). Anxiety and pain: epinephrine-induced hyperalgesia and attentional influences. Pain $76,309-316$.

Janssen, S.A. \& Arntz, A. Uncontrollable stress and opioid-mediated analgesia in novice parachute jumpers (submitted).

Janssen, S.A. \& Arntz, A. No interactive effects of naltrexone and benzodiazepines on pain during phobic fear (in press).

Kahneman, D. (1973). Attention and effort. Prentice-Hall, Englewood Cliffs, NN.J.

Kent, G. (1984). Anxiety, pain and type of dental procedure. Behaviour Research and Therapy, 2? (5) $465-469$.

Kinscheck, I.B., Watkins, L.R. \& Mayer, D.J. (1984). Fear is not critical to classically conditioned analgesia: The effects of periaqueductal grey lesions and administration of chlordiazepoxide Brain Research. 298, 33-34.

Kilo, S. Schmelz, M., Koltzenburg, M. \& Handwerker, H.O. (1994). Different patterns of hyperalgesiat 
induced by experimental inflammation in human skin. Brain, 117, 385-396.

Kinnman, E., Nygấrds, E. \& Hansson, P. (1997). Peripheral $\alpha$-adrenoreceptors are involved in the development of capsaicin induced ongoing and stimulus evoked pain in humans. Pain, 69, 79-85.

Klepac, R.K. MoDonald, M. Hauge, G. \& Dowling, J. (1980). Reactions to pain among subjects high and low in dental fear. Joumal of Behawioral Medicine, $3(4), 373-384$

Klorman, R., Weerts, Th.C. Hastings, J.E., Melamed, B.G., Lang, P. (1974). Psychometric description of some specific fear guestionmaires. Eehawior Therapy. 5, 401-409.

Koltzeniburg, M., Lundberg, L.E.R. \& Torebjork, H.E. (1992). Dynamic and static components of mechanical hyperalgesia in human hairy skin. Pain, 51, 207-219.

Lasagna, L. (1965). Drug interaction in the field of analgesic drugs. Proceedings of the Royal Society of Medicine, $58,978-983$.

Leventhal "H. (1992). I know distraction works even though it doesn"t! Health Psychology, 11 (4), 208209.

Leventhal ${ }_{1}$ H. Everhart, D. (1979). Emotion, pain, and physical illness, In C.E. Izard (Ed.) Emotions in Personality and Psychopathology (pp. 263-298). Plenum Press, N.Y.

Levine, J.D. \& Gordon, N.C. (1986). Method of administration determines the effect of naloxone on pain. Brain Research, $365,377-378$.

Levine, J.D., Gordon, N.C. Fields, H.L. (1979). Naloxone dose-dependently produces analgesia and hyperalgesia in postoperative pain. Narure, 278; 740-741.

Lewis, J.W. Cannon, J.T. \& Liebeskind, J.C. (1980). Opioid and nonopioid mechanisms of stress analgesia. Science, 208, 623-625.

Liu, M., Max, M.B., Parada, S., Rowan, J.S. \& Bennett, G.J. (1996). The sympathetic nervous system contributes to capsaicin-evoked mechanical allodyria but not pinprick hyperalgesila in humans. Journal of Neuroscience 16 (22), 7331-7335.

MacLeod, C. Mathews, A. (1988). Anxiety and the allocation of attention to threat. The Quarterly Journal of Experimental Psychology, 40A (4), 653-670.

Maier, S.F., Sherman, J.E., Lewis, J.W., Terman, G.W. \& Liebeskind, J.C. (1983). The opioid/ nonopioid nature of stress-induced analgesia and learned helplessness. Journal of Experimental Psychology, 9(1), 80-90.

Maier, S.F. (1990). Diazepam modulation of stress-induced analgesia depends on the type of analgesia. Behavioral Neuroscience, $104(2), 339-347$.

Malow, R.M., West, J.A. \& Sutker, P.B. (1987). A sensory decision theory analysis of anxiety and pain responses in chronic drug abusers. Joumal of Abnormal Psychology 96 (3), 184-189.

Malow, R.M., West, J.A. \& Sutker, P.B. (1989). Anxiety and pain response changes across treatment: sensory decision analysis. Pain, 38, 35-44.

Marks, I.M. \& Mathews, A.M. (1979). Brief standard self-rating for phobic patients. Behaviour Research and Therapy, $17,263-267$.

Marks, I.M. (1987). Fears, phoblas, and rituals: Panic, anxiety, and their disorders. Oxford University Press, N.Y.

Matzel, L.D. \& Miller "R.R. (1987). Recruitment time of conditioned opioid analgesia. Physiology \& Behavior, 39; 135-140.

McCaul, K.D. \& Haugtvedt, C. (1982). Attention, distraction, and cold pressor pain. Journal of Personality and Social Psychology. 43. 154-162.

McCaul, K.D. \& Malott, JM. (1984). Distraction and coping with pain. Psychological Bulletin, 95 (3), 516.533

McCaul, K.D., Monson, N. \& Maki, R.H. (1992). Does distraction reduce pain-produced distress among college students? Health Psychology, 11 (4), $210-217$.

McCubbin, J.A. (1993), Stress and endogenous opiolds: behavioral and circulatory interactions. Biological Psychology: 35, 91-122.

McNeil, D.W. \& Brunetti, D.G. (1992), Pair and fear: a bioinformational perspective on responsivity to imagery. Behaviour Research and Therapy, 30 (5), 513-5:20.

Melzack, R. \& Wall, P.D. (1965). Pain mechanisms: a new theory. Science, 150, 971-979.

Melzack, R. \& Wall, P.D. (1982). The challenge of pain. Penguin books Ltd., Suffolk.

Merluzzi, T.V., Taylor, C.B., Boltwood, M. Götestam, K.G. (1991). Opioid antagonist impedes exposure. Joumal of Consulting and Clinical Psychology, 59, 425-430

Nesse, R.M., Curtis, G.C., Thyer, B.A. McCann, D.S., Huber-Smith, M.J., Knopf, R.F. (1985) Endocrine and cardiovascular responses during phobic anxiety. Psychosomatic Medicine, 47 (4), 
320-332.

Nisbutt, R.E. Schachter, S. (1966). Cognitive manipullation of pain. Joumal of Experimentel Social Psychology, 2, 227-236.

Notermans, S.L.H. (1966). Measumement of the pain threshold determined by clectrical stimulation and its cilinical application. Neurology, 16, 1071-1086.

Passchier, J., Timmeman, L., Verheil, R, Tullen, J.H.M., Pepplinkhuzern, L. \& Verhage, F. (1992) Positive associations between anticipatory anxiety and needle pain for subjective bul not for physiological measures of anxiety. Psychological Reports, 70, 1059-1062.

Paul G.L. B Bernstein. D.A. (1973). Anxiety and Clinical Problems: systematic desensitization and related techniques. General Learning Press, Morristown, N.J.

Pennebaker, J.W. \& Lightner, J.W. (1980). Competition of internal and external intormation in an exercise setting. Joumal of Personality and Social Psychology, 39 (1), 165-174.

Pennebaker, JW. \& Skelton, J.A. (1978). Psychological parameters of physical symptoms . Personafty and Social Psychology Bulletin, 4 (4), 524-530.

Pitman, R.K. wan der Kolk, B.A., Or, S.P. \& Greenberg, M.S. (1990), Naloxone-reversible analgesic response to combat-related stimuli in posttraumatic stress disorder. Archives of General Pspchiatry, 47, 541-544.

Price, K. P. \& Tursky, B. (1975). The effect of varying stimutus parameters on judgments of nociceptive electrical stimulation. Psychaphysiology, 12(6), 663-666.

Reisenzein, R. (1983). The Schachter theory of emotion: two decade later. Psychological Bulletin, 94 239-264.

Reiss, S. Peterson, R.A. Gursiky, D.M. \& McNally, R.J. (1986). Anxiety sensitivity, anxiety frequency and the prediction of fearfulness. Behaviour Research and Therapy, 24, 1-8.

Roberts, W.J. \& Elardo, S.M. (1985). Sympathetic activation of a-delta nocicepltors. Somatosensory Research, 3, 33-44.

Rodgers, R.J. \& Randall, J.L. (1988). Enwironmentally induced analgesia: siturational factors, mechanisms and significance. In R.d. Rodgers \& S.ل. Cooper (Eds.) Endorphins; Opiates and Behavioural Processes, John Wiley, N.Y.

Rogers, R.W. \& Deckner, C.W. (1975). Effects of fear appeals and physiological arousal upon emotion, attitudes, and cilgarette smoking. Joumal of Porsonality and Social Psychology, 32 (2). 222 230 .

Rose, S.R. \& Orlowski, J. (1983). Review of research an endorphins and learning. Developmental and Behavioral Pediatrics, 4, 131-135.

Roth, W.T., Breivik, G. Jargenisen, P.E., Hoffman, S. (1996). Activation in nowice and expert parachutists while jumping. Psychophysiology $33 ; 63-72$.

Rotter, J.B. (1966). Generalized expectancies for internal vs. external control of reinforcement. Psychological Monographs, 80, 1-28.

Rowati, L.C., Sacerdotie, P.,Fumagalli, P, Bianchi, M. Mantegazza, P. Panerai, A.E. (19g0). Benzadiazepines and their antiagonists interfere with opioid-dependent stress-induced analgesia. Pharmacology Bhochemistry and Behawior, 36, 123-126

Roy-Byrne, P.P., Risich, S.C. \& Unde, T.W. (1988). Neuroendocime effects of diazepam in normal subjects following byief painful stress. Joumal of Chical Psychopharmacology, 8(5), 331-335.

Sanger, D.J. (1985). Minireview GABA and the behavioral effects of anxiolytic drugsi. Life Sciences: $36,1503-1513$.

Sato, J. \& Perl, E.R. (1991). Adrenergic excitation of cutaneous pain receptors induced by peripheral nerve infury. Science:, 251, 1608-1610.

Schachter S. \& Singer, J. (1962). Cognitive, social and physiological determinants of emotional state Psychological Review, 69, 379-399.

Schedlowskik, N. Flage, T., Richter, S., Tewes, U., Schmidt, R.E "Wagner, T,O.F. (1995). B. endorphin, but not substance-P, is increased by acute stress in lhumans. Psychoneuraendocrinology, 20(1), 103-110.

Schumacher, R. \& Velden, M. (1984). Anxiety, pain experience, and paim report: a signaludetection study. Perceptual and molor skills, 58, 339-349.

Shea, $V$.K. \& Per, E.R. (1985). Failure of sympathetic stimulation to affect responsiveness of rabbit polymodal nociceptors. Joumal of neurophysiology, 54(3), 513-519

Shiffrin, R.M. \& Sichneider, W. (1977). Controlled and automatic lhumarn information processing: UI. Perceptual learning, automatio attending and a general theory. Psychological Rewlew, 84, 127- 
190.

Singh, P.N. al al (1981), Clinicai evaluation of diazepam for pain relief of postoperative pain, British Journat of Anassthesialogy. 53,831836.

Splelberger, C.D. Gorsuch, R., Lusheve, R. (1970). STAl manual for the State-Trait Anxiety Inventory. Consulting Psychologists Press, Palo Alto, Callifornia.

Stacher, G. Abatzi, T. Schulte, F., Schneider, C., Stacher-Janotta, G, Gaupmann, G., Mittelbach. G. Steinninger, H. (1988). Nalloxone does not alter the perception of pain induced by electrical and thermal stimulation of the skin in healthy humans. Pain, 34, 271-276.

Tan, S. (1982). Cognitive and cognitive-behavioral methods for pain control: a selective review. Pain. $12,201-228$.

Terenius, L. (1985). Families of opioid peptides and classes of opioid receptors. In: H.L. Fields \& J.L. Besson (Eds.), Advances in pain research and therapy (9, pp. 463-477). Raven Press, N.Y.

Thyer, B.A, Matthews, J. (1986). The effect of phabic anxiety on plasma beta-endorphin: a singlesubject experiment. Behawiour Ressarch and Therapy, 24) (2), 237-241.

Tripp, G. McNaughtion, N. (1992). Schedule Dependence of the interaction of naloxone and chlordiazepoxide. Pharmacology Biochemistry and Behawor, 41, 475m-481.

Tumer, S. M., Beidel, D. C., Dancu, C. V., Stanley, M. A. (1989). An Empirically Derived Inventory to Measure Social Fears and Anxiety: The Social Phobia and Anxiety Inventory. Psychological Assessment: A Journal of Consulting and Clinical Psychology, $1,35-40$.

Turpin, G. (1986). Effects of stimulus intensity on autonomic responding: The problem of differentiating orienting and defense reflexes. Psychophysiology, 23(1), 1-14.

Ursin. H., Baade, E., Seymour "L. (1978). Psychabiology of stress: A study of coping men. Academic Press, New York.

Velten, E. (1968). A laboratory task for the induction of mood states. Behaviour Research and Therapy. 6, 473-482.

Veltman, D.J. (1995). Fear of bodily sensations, epinephrine, and panic anxiety. Dissertation, Amsterdam, VU.

Volicer, B.J. (1978). Hospital stress and patient reports of pain and physical status. Journal or Human Stress, 4. 28-37

Wall, P.D. (1979). On the relation of injury to pain. Pain, 6, 253-264.

Wardle, $\downarrow$. (1983). Psychological management of anxiety and pain during dental treatment. Journal of Psychosomatic Research, 27 (5), 399-402.

Watkins, L.IR. \& Mayer, D.J. (1982). Organization of endogenous opiate and nonopiate poain control systems. Science, 216, 1185-1192.

Weiner, N. (1985). Norepinephrine, epinephrine, and the sympathomimetic amines. In A.G. Goodman Gilman, L.S. Goodman, T.W. Rall \& F. Murad (Eds.) The Pharmacological Basis of Therapeutics (7th ed.), Macmillan, N.Y...

Weisenberg, $M_{2}$ Aviram, $O$., Wolt, $Y$. \& Raphaeli, N. (1984). Relevant and irrelevant anxiety in the reaction to pain. Pain, 20, 371-383.

Wessel I \& Merckelbach, H. (1996). The impact of anxiety on memory for details in spider phobics. Applied Cognitive Psychology, 11, 444, 1-9.

Wiesenfeld-Hallin, Z \& Hallin, R.G. (1984). The influence of the sympathetic system on mechanoreception and naciception: a review. Human Nourobiology, 3, 41-46

Willer, J.C. \& Albe-Fessard, D. (1980). Electrophysiological evidence for a release of endogenous oplates in stress-induced 'analgesia' in man. Brain Research, 198, 419-426.

Willer, J.C. \& Ernst. M. (1986). Somatovegetative changes in stress-induced analgesia in man: An electrophysiological and pharmacological study. Annals New York Academy of Sciences, 467. 256-272. 


\section{Summary}

The present thesis deals with possible psychological and physiological mediators in the influence of anxiety on pain. In particular, the role of attentional focus, endogenous opioids and epinephrine release is investigated.

Chapter 1 provides an introduction, in which theoretical and clinical views on the relationship between anxiety and pain are presented. It is argued that while pain is usually regarded as a sensation evoked by noxious stimulation, the way in which pain is experienced is usually no direct function of the degree of noxious stimulation. Anxiety is believed to importantly influence the perception of pain, and the evidence is discussed for various mechanisms that have been hypothesized to mediate this influence.

Chapter 2 presents an overview of studies with human subjects on the influence of anxiety on pain. It is demonstrated that while correlational studies often report positive correlations between anxiety and pain, experimental studies in which an anxious state was induced report both pain-increasing and paininhibitory effects of anxiety. The direction of an effect seems to depend on the focus of anxiety, with pain-related anxiety leading to an increase, and painirrelevant anxiety leading to a decrease in pain. However, in several experiments it was shown that attentional focus could explain these effects. Next to evidence for a role of attentional focus, some studies demonstrated endogenous opioidmediated pain-inhibitory effects.

Chapter 3 starts with a presentation of the research questions. Subsequently, six consecutive studies are reported in which these questions were experimentally addressed. In section 3.2 and 3.3 , spider phobic anxiety was used to investigate the relative effects of endogenous opioids and attentional focus. While prior evidence for a role of attentional focus was confirmed, no endogenous opioid effects were found in these phobic situations. Since this was attributed to the relative controllability of the situation, in section 3.4 the possibility of an endogenous opioid-mediated analgesia in humans was further investigated by a more 'flooding-like' confrontation with a spider. Again, however, no endogenous opioid effects were found, while reducing anxiety by the benzodiazepine alprazolam resulted in a slight pain inhibition. Iin section 3.5 a first-time parachute jump, an uncontrollable situation outside the lab, was chosen to study endogenous opioid effects. Indeed, subjects receiving naloxone, an opioid antagonist, showed a pain increase with respect to subjects receiving placebo. Section 3.6 addressed the question whether epinephrine, in doses that correspond to those during anxiety, exerts hyperalgesic effects. Since pain measures showed effects of epinephrine, especially when skin was sensitized, it was concluded that anxiety may lead to a pain increase through the release of epinephrine. However, effects of attention manipulation appeared to be larger. Finally, section 3.7 investigated whether anxiety may exacerbate pain because of a disturbance in attention regulation. It was hypothesized that during painirrelevant anticipatory anxiety, when the object of threat is absent, attention will 
be automatically allocated to the pain. In a stimulus-rich environment, where there is competition for attention, anxious subjects were expected to seek less distraction from pain than non-anxious subjects. Although the manipulation of anticipatory anxiety for an oral presentation did not prove successful, some evidence was found for a disturbance in attention regulation. That is, high trait socially anxious subjects reported more anxiety, stronger pain, more attention towards pain and less memory for neutral objects in the environment than the low trait socially anxious subjects.

Chapter 4 recapitulates the results of the experimental studies. An attempt is made to integrate the evidence for the various mechanisms into a preliminary model on the influence of anxiety on pain. It is argued that the degree of control that is experienced in a threatening situation determines both the neuroendocrine and the behavioural response, with a situation that is perceived as potentially controllable leading to catecholamine release and fight or flight behaviour. When the situation is perceived as uncontrollable, however, endogenous opioid release and submissive behaviour are expected, especially, it seems, when the threat involves some kind of physical harm. Only in these cases analgesic effects are expected, while in those cases in which catecholamine release is the primary neuroendocrine response pain-increasing effects may be expected. However, since hyperalgesic effects of epinephrine were very small, they may not always be noticeable, expect when the skin is sensitized or injured. Furthermore, since effects of attentional focus were much larger, it seems that epinephrine effects during anxiety may easily be overruled by the strong effects of attention when necessary, for instance to facilitate fight or flight behaviour. It is suggested that future research should be directed to the paradoxical effects of perceived control on pain. Finally, some possible implications of the results for the treatment and understanding of acute and chronic pain are discussed. 


\section{Samenvatting}

Dit proefschrift richt zich op de psychologische en fysiologische mechanismen die mogelijk een rol spelen bij de invloed van angst op piin. In het bijzonder worden onderzocht de rol van aandachtsmechanismen, de rol van endogene opiaten en de rol van adrenaline.

Hoofdstuk 1 biedt een inleiding, waarin theoretische en klinische opvattingen over de relatie tussen angst en pijn worden behandeld. Hoewel pijn gewoonlijk beschouwd wordt als een sensatie die veroorzaakt wordt door potentieel schadelijke stimuli, is de manier waarop pijn ervaren wordt meestal geen directe functie van de mate van schadelijke stimulatie. Onder andere angst zou een belangrijke invloed hebben op de waarneming van pijn. Aanwijzingen worden besproken voor verschillende mechanismen die verondersteld worden een medierende rol te spelen in deze invloed van angst op pijn.

Hoofdstuk 2 geeft een overzicht van studies naar de invloed van angst op pijn. Hieruit blijkt dat correlationele studies vaak positieve correlaties tussen angst en pijn rapporteren, terwijl experimentele studies waarin angst werd geinduceerd zowel pijnversterkende als pijndempende effecten van angst laten zien. De richting van een effect lijkt af te hangen van de richting van de angst, waarbij pijngerelateerde angst leidde tot een pijnversterking, en niet aan de pijn gerelateerde angst leidde tot een pijndemping. In een aantal experimenten werd echter aangetoond dat deze effecten beter verklaard kunnen worden door de focus van de aandacht. Naast aanwijzingen voor een rol van aandacht, werd in sommige studies ook een pijndemping aangetoond die gemedieerd werd door endogene opiaten.

Hoofdstuk 3 begint met een overzicht van de vraagstellingen. Vervolgens worden zes opeenvolgende studies gerapporteerd waarin deze vraagstellingen experimenteel werden onderzocht. In sectie 3.2 en 3.3 werd fobische angst voor spinnen gebruikt om de relatieve effecten van endogene opiaten en focus van de aandacht te onderzoeken. Reeds bestaande evidentie voor een rol van aandachtsfocus werd bevestigd, maar er werden in deze fobische situaties geen endogene opiate effecten gevonden. Omdat dit werd toegeschreven aan de relatieve controleerbaarheid van de situatie, werd in sectie 3.4 de mogelijkheid van een endogeen opiate pijndemping nader onderzocht in een meer 'flooding'achtige confrontatie met de spin. Wederom werden echter geen endogene opiate effecten gevonden, terwijl het dempen van de angst door middel van de benzodiazepine alprazolam resulteerde in een lichte pijndemping. In sectie 3.5 werd ervoor gekozen om endogene opiate effecten te bestuderen bij ean eerste parachutesprong, een oncontroleerbare situatie buiten het lab. Inderdaad bieek bij proefpersonen die de opiate antagonist naloxon toegediend kregen een pijnversterking op te treden ten opzichte van proefpersonen die een placebo kregen. Sectie 3.6 ging in op de vraagstelling of adrenaline, wanneer toegediend in doses die vergelijkbaar zijn met de niveaus tijdens angst, pijnversterkende effecten heeft. Aangezien de pijnmaten een pijnversterkend effect van adrenaline 
lieten zien, met name wanneer de huid gesensitiseerd was, werd geconcludeerd dat angst pijn zou kunnen versterken door middel van het vrijkomen van adrenaline. Echter, de effecten van aandachtsmanipulatie leken groter te zijn. Tenslotte werd in sectie 3.7 onderzocht of angst pijn zou kunnen versterken via een verstoring van de aandachtsregulatie. Verondersteld werd dat tijdens niet aan pijn gerelateerde anticipatieangst, wanneer het object van bedreiging afwezig is, de aandacht automatisch op de piin gericht wordt. In een stimulusrijke omgeving. waarin competitie geboden werd woor de richting van de aandacht, werd venwacht dat angstigle proefpersonen minder afleiding van de pijn zouden zoeken dan nietangstige proefpersonen. Hoewel de manipulatie van anticipatieangst voor een mondelinge presentatie niet succesvol bleek, werd wel enige aanwijzing gevonden voor een verstoring in aandachtsregulatie. Los van manipulatie rapporteerden hoog sociaal angstige proefpersonen meer angst, sterkere pijn, meer aandacht op de pijn en minder geheugen voor neutrale objecten in de omgeving dan lalag sociaal angstigen.

Hoofdstuk 4 geeft eerst een korte samenvatting van de onderzoeksresultaten. Hiena wordt een poging gedaan om de evidentie voor de verschillende mechanismen te integreren in een model voor de invloed van angst op pijn. Geopperd wordt dat de mate van ervaren controle in een bedreigende situatie zowel de neurohormonale reactie als het gedrag bepaald, waarbij een situatie die als potentieel controleerbaar ervaren wordt zou leiden tot het vrijkomen van catecholaminen en vecht- of vluchtgedrag. Wanneer de situatie echter wordt ervaren als oncontroleerbaar, zou het vrijkomen van endogene opiaten en onderdanig gedrag verwacht worden, met name, lijkt het, wanneer er sprake is van enige vorm van fysieke dreiging. Slechts in deze situaties wordt een endogeen opiate pijndemping verwacht, terwijl wanneer voornamelijk catecholaminen vrijkomen eerder een pijnversterking verwacht zou worden. Echter, deze hoeft niet altijd duidelijk te zijn, omdat de pijnversterking door adremaline vrij klein bleek te zijn, behalve wanneer de huid gesensitiseerd of beschadigd is. Bovendien leken de effecien van aandachtsfocus veel groter, zodat indien nodig effecten van adrenaline tijdens angst gemakkelijk overschaduwd zouden kunnen worden door de sterkere effecten van aandacht, bijvoorbeeld om vecht- of vluchtgedrag te vergemakkelijken. Gepleit wordt voor toekomstig onderzoek naar de paradoxalle effecten van ervaren controle op piin. Tenslotte wordt een aantal mogelijke implicaties van de resultaten voor de behandeling van en kennis over acute en chronische pijn besproken. 


\section{Dankwoord}

Al bijna terugkijkend op een fijne periode in Maastricht, wil ik enkele mensen bedanken die op welke manier dan ook hebben bijgedragen aan de totstandkoming van dit proefschrift.

Allereerst heb ik heel veel te danken aan mijn begeleider, Arnoud Arntz. Arnoud, je was een geweldig begeleider met veel kennis van zaken, en met de perfecte mix tussen loslaten in vertrouwen enerzijds, en betrokkenheid bij het project anderzijds. Dank voor alles wat ik van je geleerd heb.

Mijn promotor, Marcel van den Hout, ben ik dankbaar voor de vrijheid om "experimenteel" onderzoek te doen. Dank voor je enthousiasme tijdens het parachute-experiment en voor het op onnavolgbare wijze lospeuteren van apparatuur bij huiverige uitleners.

Gedurende de lange barre tijden in het lab en de hectische weekenden op het parachutecentrum was ik blij er niet alleen voor te staan. Artsen Nicolie Brüll, Bas Geerdes en Cees van Leeuwen, en studentes Karin Pool, Sabine Bouts, Marian van Stekelenburg en Wieke Pasman: dank voor jullie enorme inzet en de fijne samenwerking. En wanneer het even dreigde spaak te lopen in het lab waren het technisch vernuft van Theo van Aerts en het heldere inzicht van Peter de Jong onontbeerlijk. Prof. Struijker Boudier, Harrie Kurvers, Jo de Mey en Dick Veltman ben ik erkentelijk voor hun nuttige adviezen tijdens de opzet van de experimenten. Paracentrum Zwartberg wil ik bedanken voor de gastvrijheid, met name instructice Sabine Gijzen, door wie de hele boel van de grond is gekomen. Verder wil ik alle dappere proefpersonen bedanken: van spinfobici tot parachutespringers waren het stuk voor stuk helden.

De collega's van de vakgroepen Medische Psychologie en Differentiële en Experimentele Psychologie zorgden ervoor dat ik altijd met plezier ging werken. Voor een groot deel was dit ook te danken aan Marie-Anne Haenen, kamergenote en klankbord. M-A, dank je wel voor alle warmte, gesprekken en dropjes. Paranimfen Sabine Kroeze en Bert Hoekzema, fijn dat jullie me straks bij willen staan in mijn zwaarste uur. Jammer dat we binnenkort niet meer zo gemakkelijk samen het Limburgse heuvelland in kunnen fietsen. Anja van den Hout (ook zo'n fietserd) en Johan Vlaeyen bedankt voor de leuke dagen tijdens het pijncongres in Barcelona. Verder wil ik alle (FR)AlO's uit Maastricht en alle andere promovendi van de onderzoeksschool Experimentele Psychopathologie bedanken voor het gevoel, ondanks individuele projecten, vooral samen aan het promoveren te zijin.

Rest mij het thuisfront. Mijn ouders ben ik dankbaar voor het aanwakkeren van mijn wetenschappelijke interesse en voor het vertrouwen. Vincent, je hebt ondanks de enorme afstand steeds aan mijn zijde gestaan, waarvoor grote dank. 


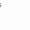




\section{Curriculum Vitae}

Sabine Anne Janssen werd op 29 juli 1970 geboren in Delft. In 1988 behaalde zij haar WWO-diploma aan het Erasmus College te Zoetermeer, waarna zij psychologie ging studeren aan de Rijksuniversiteit Leiden. Tijdens haar studie liep zij stage bij het Laboratory of Physiology in Oxford en bij de vakgroep Psychonomie van de Universiteit van Amsterdam. Op 23 februari 1994 studeerde zij cum laude af in de Psychologische Functielleer. Aansluitend werd zij per 1 maart 1994 aangesteld als Onderzoeker in Opleiding bij de vakgroep Medische Psychologie van de Universiteit Maastricht, waar het onderhavige onderzoek werd uitgevoerd en opgeschreven. Vanaf 1 september 1998 is zij werkzaam als wetenschappelijk projectmedewerker (post-doc) bij de vakgroep Klinische en Gezondheidspsychologie van de Rijksuniversiteit Leiden. Hier zal zij het onderzoek naar pijn en somatisatie voortzetten. 